Tsubasa Yamauchi

Control chart for monitoring the ratio of two Poisson rates

São Paulo, Brazil 

Tsubasa Yamauchi

\title{
Control chart for monitoring the ratio of two Poisson rates
}

\author{
Dissertation presented to Polytechnic School \\ of University of São Paulo to obtain the Mas- \\ ter in Science
}

University of São Paulo - USP

Polytechnic School

Graduate Program in Production Engineering

Adviser: Linda Lee Ho

São Paulo, Brazil

2019 
Autorizo a reprodução e divulgação total ou parcial deste trabalho, por qualquer meio convencional ou eletrônico, para fins de estudo e pesquisa, desde que citada a fonte.

Este exemplar foi revisado e alterado em relação à versão original, sob responsabilidade única do autor e com a anuência de seu orientador.

São Paulo, 26 de Setembro de 2019

Assinatura do autor

Assinatura do orientador

Tsubasa Yamauchi

Control chart for monitoring the ratio of two Poisson rates/ Tsubasa Yamauchi. - Ver.

Corr. - São Paulo, Brazil, 2019-

$125 \mathrm{p}$.

Adviser: Linda Lee Ho

dissertation (Master) - University of São Paulo - USP

Polytechnic School

Graduate Program in Production Engineering, 2019.

1. The ratio of two Poisson rates 2. Normal transformation I. Linda Lee Ho. II. University of São Paulo. III. Polytechnic School. IV. Master Degree 


\section{Acknowledgements}

First, and foremost, I would like to thank my advisor, Dr. Linda Lee Ho, for helping me develop the skills and keeping believing me. Your guidance, encouragement, support and especially patience are greatly appreciated.

I thank the members of my committee, Dr. Marcela Machado, Dr. Roberto da Costa Quinino, Dr. Carla Almeida Vivacqua, Dr. Airlane Alencar, Dr. André Luís Santos for their indications to improve my work.

I also express my gratitude to every professor who gave lectures, Dr. Afonso Carlos Correa Fleury, Dr. Celma de Oliveira Ribeiro, Dr. Hugo Tsugunobu Yoshida Yoshizaki, Dr. Maria Elena Santos Taqueda, the school and staff.

Brazil, I should thank you so much for giving me challenge, opportunity to learn new knowledge, different culture and way of thinking.

Finally, I would like to thank my entire family for their support and giving me liberty to do what I want to do. 



\section{Abstract}

In this study we are concerning in monitoring the ratio $\rho$ of two Poisson rates by the control chart. Let $X$ and $Y$ be two independent Poisson random variables with means $\lambda_{1}$ and $\lambda_{2}=\lambda_{1} / \rho$ respectively. The study considers that only individual observations $X_{i}$ and $Y_{i}$ are available at each sampling time $i$. The performance in detecting shifts on the ratio $\rho$ by several statistics, some based on normalized transformations are evaluated by an extensive simulation study. Two types of control charts, Shewhart and EWMA are considered. The one-sided control limit with UCL is applied so that we are focusing on detecting when the ratio $\rho$ shifted to higher rate in this dissertation. Our focus is on monitoring the ratio $\rho$ and not on the inference of means of $\lambda_{1}$ and $\lambda_{2}$. The results pointed out that EWMA control chart is better alternative. Some guidelines indicating which statistics yield best performance are proposed for the practitioners. In general, the combination of statistics $W_{0}$ and $Z_{4}^{* *}$ are recommended once EWMA control chart is applied. In case the difference between $\lambda_{1}$ and $\lambda_{2}$ is small, the statistic $W_{4}^{*}$ could be used instead of $W_{0}$. Numerical example illustrates an application.

Keywords: Maximum likelihood estimator; conditioned maximum likelihood estimator; normal transformations; EWMA; Shewhart control charts; Monte Carlo simulation. 



\section{Resumo}

Neste estudo estamos interessados no monitoramento da razão $\rho$ de duas variáveis de Poisson por meio de gráfico de controle. Definimos $X$ e $Y$ como variáveis aleatórias independentes Poisson de parâmetros $\lambda_{1}$ e $\lambda_{2}=\lambda_{1} / \rho$ respectivamente. O estudo considera que apenas observações individuais $X_{i}$ e $Y_{i}$ estão disponíveis de cada tempo $i$. Os desempenhos de detecção de mudanças da razão $\rho$ são feitas empregando várias estatísticas, algumas são baseadas em transformações normais e foram avaliadas através uma extensa simulação. Dois tipos de gráfico de controle, Shewhart e EWMA são considerados. O limite de controle unilateral LCS é determinado visto que estamos focando na detecção da razão $\rho$ para uma taxa maior nesta dissertação. Nosso foco é no monitoramento de razão $\rho$ e não na inferência de médias $\lambda_{1}$ e $\lambda_{2}$. Os resultados apontaram que o gráfico de controle de EWMA é a melhor alternativa. Algumas orientações com indicação de estatísticas que têm os melhores desempenhos são propostas para praticantes. Em geral, a combinação de das estatístcas $W_{0}$ e $Z_{4}^{* *}$ é recomendada quando o gráfico de controle de EWMA é utilizado. No caso da diferença entre $\lambda_{1}$ e $\lambda_{2}$ for pequena, a estatística $W_{4}^{*}$ poderia ser utilizada ao invés de $W_{0}$. Um exemplo numérico ilustra uma aplicação.

Palavras-chaves: Estimador de máxima verossimilhança; Estimador condicionado de máxima verossimilhança; Transformações normais; EWMA; Gráfico de controle de Shewhart; Simulação de Monte Carlo. 



\section{List of Figures}

Figure 1 - Shewhart Control Chart . . . . . . . . . . . . . 18

Figure 2 - Simulation Flow Chart for stage I . . . . . . . . . . . . . . . . . 29

Figure 3 - Simulation Flow Chart for stage $I I \ldots \ldots$. . . . . . . . . . . 30

Figure 4 - EWMA-type Control Chart with $Z 4^{* *}$ for numerical example . . . . . . 47 



\section{List of Tables}

Table 1 - A summary of the statistics . . . . . . . . . . . . . . 31

Table 2 - Shewhart Control chart $-\lambda_{10}=7$ and $\lambda_{20}=10 \ldots \ldots$. . . . . 33

Table 3 - Shewhart Control chart $-\lambda_{10}=28$ and $\lambda_{20}=40 \ldots \ldots$. . . . . . 34

Table 4 - Shewhart Control chart $-\lambda_{10}=4$ and $\lambda_{20}=10 \ldots \ldots$. . . . . . 35

Table 5 - Shewhart Control chart $-\lambda_{10}=16$ and $\lambda_{20}=40 \ldots$. . . . . . 37

Table 6 - EWMA Control chart $-\lambda_{10}=7$ and $\lambda_{20}=10 \ldots \ldots$. . . . . . 38

Table 7 - EWMA Control chart $-\lambda_{10}=28$ and $\lambda_{20}=40 \ldots \ldots$. . . . . . 39

Table 8 - EWMA Control chart $-\lambda_{10}=4$ and $\lambda_{20}=10 \ldots \ldots$. . . . . . 40

Table 9 - EWMA Control chart $-\lambda_{10}=16$ and $\lambda_{20}=40 \ldots \ldots$. . . . . . 41

Table 10 - List of the statistics with best performance- case $\rho_{0}=0.4 \ldots \ldots$. . . 43

Table 11 - List of the statistics with best performance - case $\rho_{0}=0.7 \ldots \ldots 44$

Table 12 -Example conditions and results . . . . . . . . . . . . . . 46 



\section{Contents}

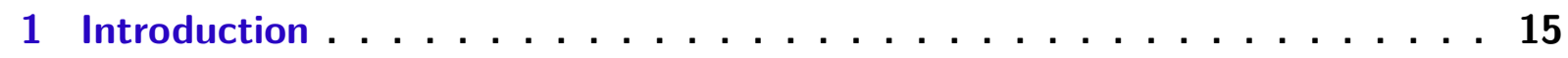

2 A brief review of the main concepts $\ldots \ldots \ldots \ldots \ldots$

2.1 Statistical process control and control charts . . . . . . . . . 17

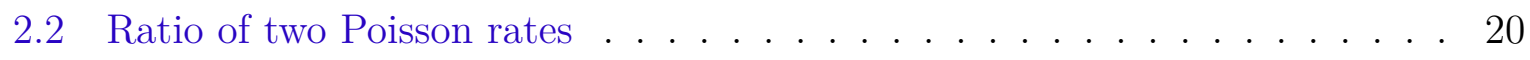

2.3 Some adjustments . . . . . . . . . . . . . . . . . . . 23

2.4 Normal transformation of Poisson variables . . . . . . . . . . . . 24

3 Methodology . . . . . . . . . . . . . . . . . 27

3.1 Monte Carlo simulation study . . . . . . . . . . . . . . . . . . . 27

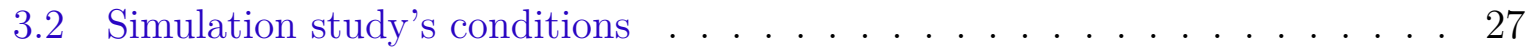

3.3 Simulation flow chart . . . . . . . . . . . . . . . . 28

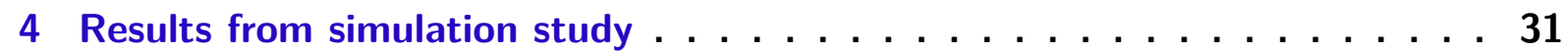

4.1 Shewhart control chart's performances . . . . . . . . . . . . . 32

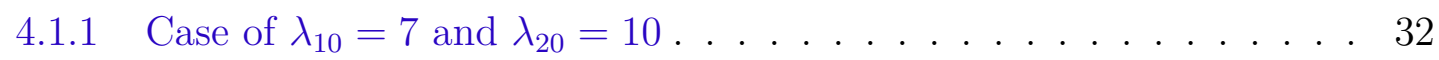

4.1 .2 Case of $\lambda_{10}=28$ and $\lambda_{20}=40 \ldots \ldots \ldots \ldots \ldots \ldots \ldots \ldots \ldots \ldots$

4.1.3 Case of $\lambda_{10}=4$ and $\lambda_{20}=10 \ldots \ldots \ldots \ldots \ldots \ldots \ldots \ldots \ldots \ldots$

4.1.4 Case of $\lambda_{10}=16$ and $\lambda_{20}=40 \ldots \ldots \ldots \ldots \ldots \ldots \ldots \ldots$

4.2 EWMA control chart's performance . . . . . . . . . . . 36

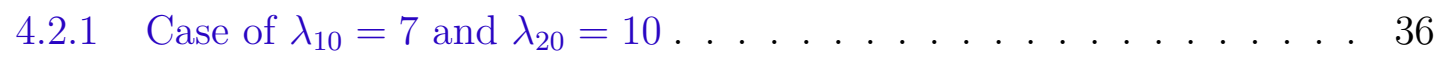

4.2 .2 Case of $\lambda_{10}=28$ and $\lambda_{20}=40 \ldots \ldots \ldots \ldots \ldots$

4.2 .3 Case of $\lambda_{10}=4$ and $\lambda_{20}=10 \ldots \ldots \ldots \ldots \ldots \ldots$

4.2 .4 Case of $\lambda_{10}=16$ and $\lambda_{20}=40 \ldots \ldots \ldots \ldots \ldots$

4.3 Overview of results $\ldots \ldots \ldots \ldots \ldots \ldots \ldots$

5 Numerical Example . . . . . . . . . . . . . . . . . . . 45

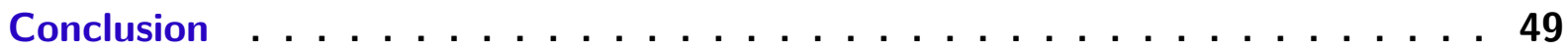

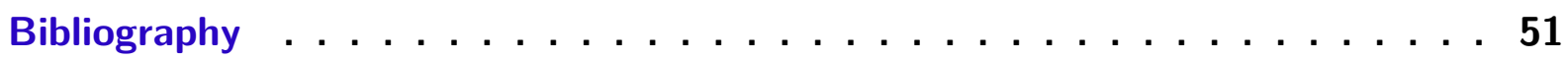

$\begin{array}{ll}\text { Appendix } & 53\end{array}$

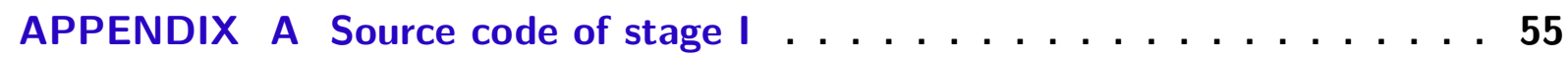

APPENDIX B Source code of stage $\| \ldots \ldots \ldots \ldots \ldots \ldots$ 



\section{Introduction}

Many companies employ indexes to monitor their activities and some of these indexes are ratio of two independent counts. The decision if an activity is stable is based on a threshold usually chosen without statistical criterion but only considering historical data and managers' expertise.

Several examples illustrate the necessity of this type of monitoring. For example, a printing machine sales company is used to planning the purchase order amount of consumables (toner, developer, drum etc.), by the monthly indicator which is estimated by the ratio between the demand of specific consumable (the amount of delivery of specific consumable from warehouse) and the MIF (the amount of printing "Machine In Field"). This indicator estimates the demand of specific consumable per a unit of machine and it should be stable if there is no extraordinary situation such as the hike (reduction) of the print volume of customer's machine, new sales of machine to the customer which has intensive production and so on. If a company could not perceive a shift of indicator, the purchase order amount would be wrong and there is the risk for the lack or excess of stock. So the monitoring of this indicator is crucial and there is little meaning to monitor individually the demand and the MIF as it is normal that the demand of the products increases (decreases) when MIF increases (decreases).

A second example is the monitoring of quality of product like cloth (linen, silk, etc). Two independent types of non-conformity are counted. Important to mention that the presence of the non-conformity does not mean that the product is inappropriate for use or prejudice the visual appearance. An increase in the ratio may harm the visual appearance like non-conformity of type 1 being more standing out than type 2 .

The two previous cases are examples which two quality characteristics are monitored simultaneously on the sample unit. Now the third example, which quality characteristic is taken on independent sample units. Let us consider two brands of a product and the brand $\mathrm{A}$ is the reference one. The producer of brand $\mathrm{A}$ would like to affirm that competitor brand $\mathrm{B}$ is $(1-\rho) \%$ worse than $\mathrm{A}$ in some quality characteristic of interest that follows Poisson distribution (like the number of breaks, faults). The company of the reference brand has interest in evaluating if the ratio of the number of defects (breaks, faults, etc) in samples of two brands remains stable.

Here we are considering that the quality characteristics $X \sim P\left(\lambda_{1}\right)$ and $Y \sim$ $P\left(\lambda_{2}=\lambda_{1} / \rho\right)$ are independent Poisson random variables. Moreover, the most interest concerns is to monitor if the ratio $\rho$ remains stable at a fixed value $\rho_{0}$, but not rather than their means. It is also assumed that at every inspection time $i$, only one observation 
of $X_{i}$ and $Y_{i}$ are available and based on them, the person in charge has to decide if the ratio $\rho$ is stable or not.

Two types of control chart are considered: Shewhart and EWMA. The performance of several statistics are compared in terms of the speed to detect shifts on parameter of interest, based on extensive simulation study, since the development of the probability distribution function of the monitored statistics are quite complicated.

This dissertation is organized as follows. In Chapter 2, a brief review of the main concepts related to control chart is presented as also the monitored statistics. Chapter 3 presents the methodology of Monte Carlo simulation and simulation conditions. Chapter 4 shows the results of the simulation and the performances of each control chart are discussed. Chapter 5 illustrates the proposal by a numerical example. At the last the conclusion, final remarks and some issues for future work are described.

The results of this dissertation are parts of a manuscript submitted for publication at Quality and Reliability Engineering International on June 24th 2019 and accepted on August 22nd 2019. 


\section{A brief review of the main concepts}

In this Chapter 2, a brief review of the main concepts related to control chart is presented. The Section 2.1 introduces the statistical process control and the control charts, Shewhart control chart and EWMA control chart. In the Section 2.2, the Poisson random variables are defined and several statistics of the ratio of Poisson rates are presented. The section 2.3 shows adjustments for some statistics. Finally some normal transformations are presented in the Section 2.4 in order to apply them for Poisson rate monitoring.

\subsection{Statistical process control and control charts}

Statistical process control (SPC) is a method to improve the manufacturing process performance and reduce variability in key parameters with statistical tools, basically histogram, check sheet, Pareto chart, cause-and-effect diagram, defect concentration diagram, scatter diagram, DOE (design of experiments) and then control chart (MONTGOMERY, 2005). Statistical methods and their application in quality improvement have developed since 1930s. In 1924, Walter A. Shewhart (SHEWHART, 1924) of Bell Laboratories introduced the statistical control-chart concept, which is considered the formal beginning of statistical quality control. The control chart has been considered as the most technically sophisticated type of online statistical process control tool to monitor the variability and to detect the shift of the key parameters such as mean and variance. After that, the acceptance sampling methodology is developed and refined as an alternative to 100 percent inspection by H.F. Dodge and H.G. Romig at same laboratory (DODGE H. F.; ROMIG, 1929). The use of quality improvement techniques, which includes control chart has promoted this tool for all type of products and services.

The control chart contains a center line that represents the average value of the key parameters and two horizontal lines, the upper control limit (UCL) and the lower control limit (LCL) as it is shown in Figure 1.

As long as the points fall within the control limits, we assume that the process is in control and no action is necessary. On the other hand, once a point falls outside of the control limits we interpret that the process is out of control and some action to find and eliminate the assignable cause should be done.

Let $X^{*}$ be a statistic, which its mean and standard deviation are respectively $\mu_{X^{*}}$ and $\sigma_{X^{*}}$, then the center line, the upper control limit and the lower control limit are defined as: 


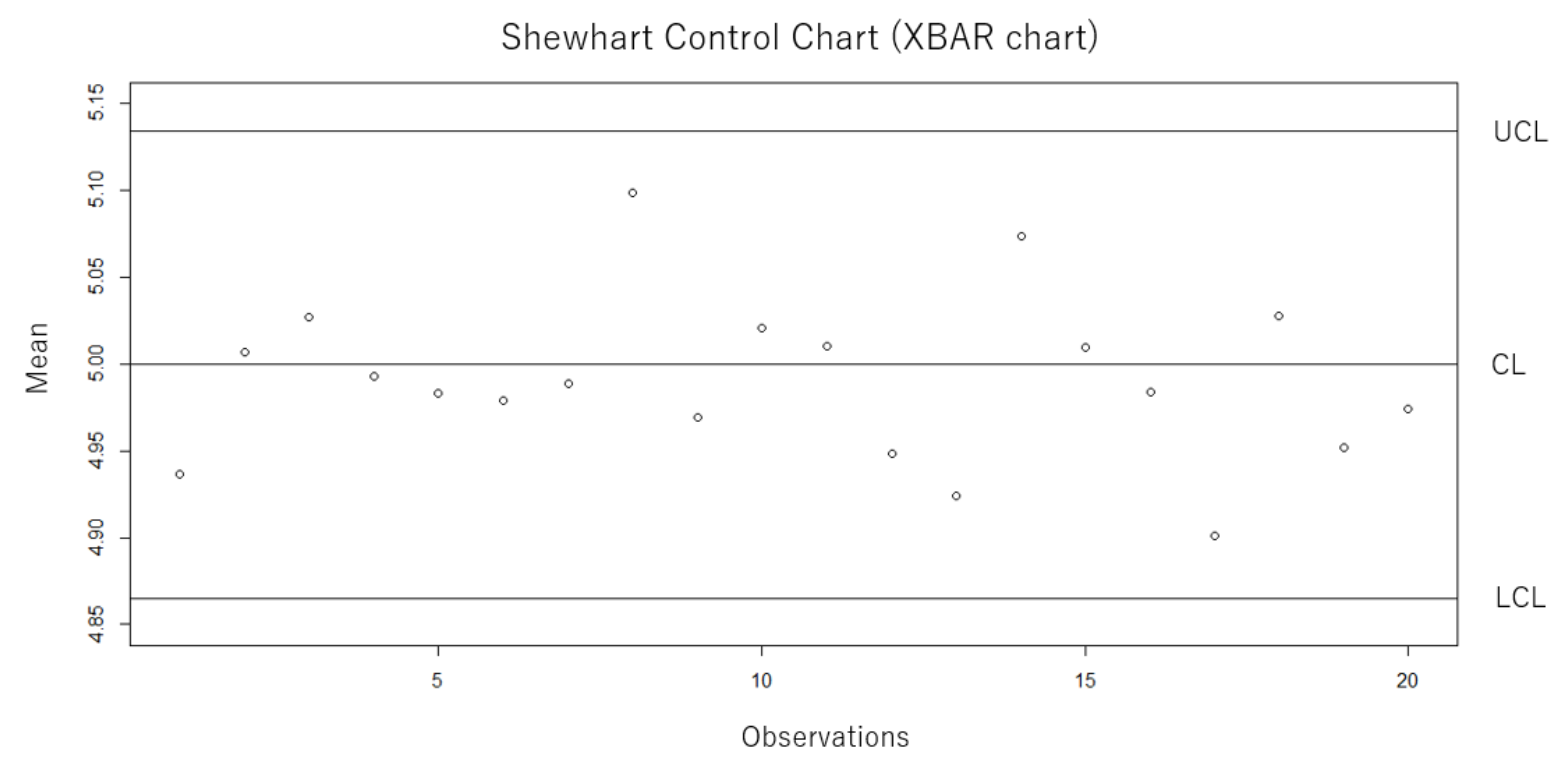

Figure 1 - Shewhart Control Chart

$$
\begin{aligned}
U C L & =\mu_{X^{*}}+L \sigma_{X^{*}} \\
C L & =\mu_{X^{*}} \\
L C L & =\mu_{X^{*}}-L \sigma_{X^{*}}
\end{aligned}
$$

where $L$ is the distance of control limits from center line by standard deviation units. Thus, whenever $X^{*}>U C L$ or $X^{*}<L C L$, the process is declared out-of-control and the search for assignable cases is started. $L$ is fixed with the consideration of type I error which is the risk of a point falling beyond the control limits when no assignable case is present and type II error which is the risk of a point falling between the control limits when the process is out of control. This primary control chart is named Shewhart control chart.

Shewhart control chart is efficient to detect large shifts in the monitored parameters. However, Shewhart control chart is based on the current value of the statistic, meaning that it uses only the information of the last sample observation. So it ignores any information given by the entire sequence of points. This fact makes Shewhart control chart insensitive to small shifts.

In 1960s, other types of control charts were introduced to cover this disadvantage of Shewhart control chart. E. S. Page developed the cumulative sum (CUSUM) control chart (PAGE, 1954) and S. Roberts introduced the exponentially weighted moving average (EWMA) control chart (ROBERTS, 1959).

Both control charts can be alternatives to the Shewhart control chart when we are interested in detecting small shifts. The performance of the CUSUM control chart and EWMA control chart are similar but EWMA control chart is considered easier to set up and operate. 
The exponentially weighted moving average (EWMA) is defined with sample statistic $X_{j}^{*}$ as the following expression, $j$ is number of observation sequence.

$$
Z_{j}=\theta X_{j}^{*}+(1-\theta) Z_{j-1}
$$

where $0<\theta<1$ is a constant and $Z_{0}=\mu_{0}$ or $Z_{0}=\bar{X}$. The center line is $\mu_{0}$ and upper and lower control limits are as follows:

$$
\begin{aligned}
& U C L_{j}=\mu_{0}+L \sigma \sqrt{\frac{\theta}{(2-\theta)}\left[1-(1-\theta)^{2 j}\right]} \\
& L C L_{j}=\mu_{0}-L \sigma \sqrt{\frac{\theta}{(2-\theta)}\left[1-(1-\theta)^{2 j}\right]}
\end{aligned}
$$

Whenever $Z_{j}>U C L_{j}$ or $Z_{j}<L C L$ the process is declared out-of-control. The factor $L$ in (2.3)-(2.4) is the distance of control limits from center line by standard deviation units. The constant $\theta$ and the factor $L$ should be selected by optimization to achieve the desired average run length (ARL).

The performance of control charts is described by the shift detection speed. This speed is usually expressed by average run length (ARL) which is the average number of points that must be plotted before a point indicates an out-of-control condition. When the monitored statistics $X^{*}$ are independent random variables, the ARL can be calculated from:

$$
A R L=\frac{1}{p}
$$

where $p$ is the probability that any point exceeds the control limits.

The in-control $A R L_{0}$ is expressed as the following, with the risk $\alpha$ of Type I error, which is the risk in a statistical test that a null hypothesis will be rejected when no difference actually existed. That is

$$
A R L_{0}=\frac{1}{\alpha}
$$

So it is better for the control chart to be set up with higher $A R L_{0}$, it means that there is lower $\alpha$, so that it is able to diminish false alarms on the control chart. On the other hand, the out-of-control $A R L_{1}$ is defined with the risk $\beta$ of Type II error as:

$$
A R L_{1}=\frac{1}{1-\beta}
$$

$\beta$ is the risk that the data on a control chart indicate the process is in control but in reality the process is out of control. The power of control chart is defined as Power $=1-\beta$. Then $A R L_{1}$ is better to be lower as the risk $\beta$ is lower. However when the monitored statistics 
$X^{*}$ are not independent (for example like (2.2)), $A R L$ can be achieved by Markov Chain approach as proposed by Brook and Evans (BROOK; EVANS, 1972) or by simulation of the run length distribution.

However there is a trade-off between $\alpha$ and $\beta$, as lower $\alpha$ increases $\beta$ and lower $\beta$ increases $\alpha$. So our purpose is to look for sufficiently high $A R L_{0}$ in order not to detect false alarm of Type I error and at the same time low $A R L_{1}$ to detect the shift as possible as rapid.

In process monitoring, there are phases I and II to apply control charts. Phase I is the first step to bring the process into a state of statistical control. In phase I, set of process data is gathered and analyzed and then trial control limits are constructed to determine if the process has been in control over the period of time where the data were collected and to see if reliable control limits can be established to monitor future process.

In phase II the control chart is applied to monitor the process by comparing the sample statistic for each successive sample. The process is assumed to be reasonable stable. ARL is a valid basis for evaluating the performance of a control chart in phase II.

The focus of this study is on phase II to monitor the process by the ratio of two Poisson rates.

\subsection{Ratio of two Poisson rates}

Poisson distribution is the probability distribution that expresses the probability of the number of random occurrences of events in a fixed interval of time or space.

Let $\lambda$ be the average rate of event, $n(0 \leq n<\infty)$ the number of events and $t(0 \leq t<\infty)$ the length of the interval time, the probability to observe $n$ events is:

$$
p_{n}(t)=\frac{(\lambda t)^{n} \cdot e^{-\lambda t}}{n !}
$$

In this study a time interval $t$ is considered as unity, so the expression is simplified with:

$$
p_{n}=\frac{(\lambda)^{n} \cdot e^{-\lambda}}{n !}
$$

The counting process $\{N(t), t \in[0, \infty)\}$ is called a Poisson process with rates $\lambda$ with the following conditions:

1. $N(0)=0$

2. $N(t)$ has independent increments 
3. The number of events has Poisson distribution

Let two independent Poisson random variables $X$ and $Y$ be the corresponding number of events $X \sim \operatorname{Poisson}\left(\lambda_{1}\right)$ and $Y \sim \operatorname{Poisson}\left(\lambda_{2}\right)$ with time interval $t=1$.

Our interest is one-sided hypotheses, the ratio of Poisson rate is equal to a specified average rate $\rho_{0}$ against greater than $\rho_{0}$ :

$$
H_{0}: \lambda_{1} / \lambda_{2}=\rho_{0} \text { against } H_{1}: \lambda_{1} / \lambda_{2}>\rho_{0}
$$

The applied control chart in this study is to detect the shift of hypotheses $H_{1}$. The maximum likelihood estimate (MLE) of $\lambda_{1}$ and $\lambda_{2}$ given single observations of $X_{t}$ and $Y_{t}$ are respectively:

$$
\widehat{\lambda}_{1}=\frac{X_{t}}{t} \text { and } \widehat{\lambda}_{2}=\frac{Y_{t}}{t}
$$

where $t$ is the interval of time. Thus, the MLE of $\rho$ with individual observations $X$ and $Y$ is:

$$
\widehat{\rho}=\frac{X}{Y}
$$

As the determination of the distribution (2.11) is quite complicated, the first candidate that comes to our mind is to consider

$$
W_{0}=\widehat{\lambda}_{1}-\widehat{\lambda}_{2}=X-Y
$$

as a monitored statistic to detect shifts in the ratio $\rho$.

According to $\mathrm{Gu}$ et al. (GU K.; NG, 2008), the constrained maximum likelihood estimates (CMLE) of $\lambda_{1}$ and $\lambda_{2}$ can be shown to be:

$$
\widehat{\lambda}_{1}=\frac{X+Y}{1+1 / \rho_{0}} \text { and } \widehat{\lambda}_{2}=\frac{X+Y}{1+\rho_{0}} .
$$

Additionally, they showed that the null hypothesis of (2.9) can be re-expressed as $H_{0}$ : $\lambda_{1}-\rho_{0} \lambda_{2}=0$ so the test statistics based on the statistic

$$
W_{0}^{1}=\widehat{\lambda}_{1}-\rho_{0} \widehat{\lambda}_{2}
$$

can be a monitored statistic. The variance of the statistic $W_{0}^{1}$ is given by:

$$
\sigma_{W}^{2}=\frac{\lambda_{1}}{t}+\frac{\rho_{0}^{2} \lambda_{2}}{t}
$$


Considering $t=1$, the test statistic $W_{0}^{1} / \sigma_{W}$ for $H_{0}$, replaced by MLEs of (2.10), we get:

$$
W_{1}=\frac{X-\rho_{0} Y}{\sqrt{\lambda_{1}+\rho_{0}^{2} \lambda_{2}}}
$$

In case of $\lambda_{1}$ and $\lambda_{2}$ unknown, $\sigma_{W}^{2}$ can be estimated by:

$$
s_{W}^{2}=\widehat{\lambda}_{1}+\rho_{0}^{2} \widehat{\lambda}_{2}
$$

and the test statistic $W_{0}^{1} / s_{W}$ for $H_{0}$, replaced by MLEs of (2.10) becomes:

$$
W_{1}^{*}=\frac{X-\rho_{0} Y}{\sqrt{X+\rho_{0}^{2} Y}}
$$

Similarly, replacing CMLE's of (2.13), we obtain:

$$
W_{2}^{*}=\frac{X-\rho_{0} Y}{\sqrt{(X+Y) \rho_{0}}}
$$

And a new statistic can be derived from by a replacement of expected rate of $\mathrm{X}$ and $\mathrm{Y}$ in the denominator:

$$
W_{2}=\frac{X-\rho_{0} Y}{\sqrt{\left(\lambda_{1}+\lambda_{2}\right) \rho_{0}}}
$$

Furthermore, Gu et al. (GU K.; NG, 2008) rewrote the null hypothesis (2.9) as $H_{0}$ : $\ln \left(\lambda_{1} / \lambda_{2}\right)-\ln \left(\rho_{0}\right)=0$. The test statistic based on the statistic $U=\ln \left(\widehat{\lambda}_{1} / \widehat{\lambda}_{2}\right)-\ln \left(\rho_{0}\right)=$ $\ln (X / Y)-\ln \left(\rho_{0}\right)$ can be established replacing by MLEs. The variance of $U$ is approximated by:

$$
\sigma_{U}^{2} \approx \frac{1}{\lambda_{1}}+\frac{1}{\lambda_{2}}
$$

Hence, the following statistic can be used to monitor $\rho$

$$
W_{3}=U / \sigma_{U}=\frac{\ln (X / Y)-\ln \left(\rho_{0}\right)}{\sqrt{1 / \lambda_{1}+1 / \lambda_{2}}}
$$

When $\sigma_{U}^{2}$ are unknown it can be estimated as

$$
s_{U}^{2} \approx \frac{1}{\hat{\lambda}_{1}}+\frac{1}{\widehat{\lambda}_{2}}
$$

Thus, the test statistic $U / s_{U}$ are proposed by Gu et al. (GU K.; NG, 2008). After replacing by their MLEs it results:

$$
W_{3}^{*}=\frac{\ln (X / Y)-\ln \left(\rho_{0}\right)}{\sqrt{1 / X+1 / Y}}
$$


Or replacing by CMLEs, it yields:

$$
W_{4}^{*}=\frac{\ln (X / Y)-\ln \left(\rho_{0}\right)}{\sqrt{\left(2+1 / \rho_{0}+\rho_{0}\right) /(X+Y)}}
$$

Again new statistic can be proposed from (2.22) replacing $X$ and $Y$ by their expected rates in the denominator as

$$
W_{4}=\frac{\ln (X / Y)-\ln \left(\rho_{0}\right)}{\sqrt{\left(2+1 / \rho_{0}+\rho_{0}\right) /\left(\lambda_{1}+\lambda_{2}\right)}}
$$

\subsection{Some adjustments}

Some statistics used to monitor are presented in the previous section. To avoid division by zero, the addition of 1 for denominator is considered. For example the statistic (2.11) is reexpressed as

$$
\widehat{\rho}_{s d}^{*}=\frac{X}{Y+1}
$$

In this case our interest indicator, the ratio of Poisson rate $\rho_{0}$ is transformed to another ratio of Poisson rate $\rho_{0} *$ and the hypotheses test (2.9) is re-expressed as below:

$$
H_{0}: \lambda_{1} /\left(\lambda_{2}+1\right)=\rho_{0} * \text { against } H_{1}: \lambda_{1} /\left(\lambda_{2}+1\right)>\rho_{0} *
$$

Some adjustments are made for the other statistics as following: For the statistic (2.16),

$$
W_{1}^{* *}=\frac{X-\rho_{0} Y}{\sqrt{X+\rho_{0}^{2} Y+1}}
$$

For the statistic (2.2),

$$
W_{2}^{* *}=\frac{X-\rho_{0} Y}{\sqrt{(X+Y) \rho_{0}+1}}
$$

For the statistic (2.19) and (2.21),

$$
\begin{aligned}
W_{3}^{\star} & =\frac{\ln [(X+1) /(Y+1)]-\ln \left(\rho_{0}\right)}{\sqrt{1 / \lambda_{1}+1 / \lambda_{2}}} \\
W_{3}^{* *} & =\frac{\ln [(X+1) /(Y+1)]-\ln \left(\rho_{0}\right)}{\sqrt{1 /(X+1)+1 /(Y+1)}}
\end{aligned}
$$

And finally for the statistic (2.22) and (2.23),

$$
\begin{gathered}
W_{4}^{\star}=\frac{\ln [(X+1) /(Y+1)]-\ln \left(\rho_{0}\right)}{\sqrt{\left(2+1 / \rho_{0}+\rho_{0}\right) /\left(\lambda_{1}+\lambda_{2}\right)}} \\
W_{4}^{* *}=\frac{\ln [(X+1) /(Y+1)]-\ln \left(\rho_{0}\right)}{\sqrt{\left(2+1 / \rho_{0}+\rho_{0}\right) /(X+Y+1)}}
\end{gathered}
$$




\subsection{Normal transformation of Poisson variables}

In the literature many transformations have been proposed for Poisson variables in order to get approximately normal variables. On the purpose of the control chart performance improvement, the following normal transformations are applied separately on each the Poisson variables. They are applied on (2.11) or on (2.24). Here we list the transformations considered in this study

(a) Bartlett's Transformation (BARTLETT, 1936)

The square root transformation is applied on $X$ and $Y$ for the statistic (2.24) in order avoid the denominator be zero. So the ratio with this transformation in this study is expressed as below:

$$
\hat{\rho}_{s q}^{*}=\frac{\sqrt{X}}{\sqrt{Y+1}}
$$

As $\sqrt{X}$ and $\sqrt{Y}$ are $\approx N(0 ; 1 / 2)$, this transformation can be applied for the statistic (2.14). After some manipulations, the final statistic can be written as.

$$
Z_{1}=\frac{2\left[\sqrt{X}-\sqrt{\rho_{0} Y}\right]}{\sqrt{1+\rho_{0}}} \approx N(0,1)
$$

(b) Anscombe's Transformation (ANSCOMBE, 1948)

The transformation proposed by Anscombe is also a square root plus a constant equal to $3 / 8$ and is applied on (2.11). Then the transformation results in:

$$
\hat{\rho}_{a s}=\sqrt{\frac{X+\frac{3}{8}}{Y+\frac{3}{8}}}
$$

Huffman (HUFFMAN, 1984) proposed to apply Anscombe's transformation on statistics $W_{0}^{1}=\widehat{\lambda}_{1}-\rho_{0} \widehat{\lambda}_{2}$ in order to reduce the variance of the statistic. This statistic is expressed by:

$$
Z_{2}=\frac{2\left[\sqrt{X+3 / 8}-\sqrt{\rho_{0}(Y+3 / 8)}\right]}{\sqrt{1+\rho_{0}}}
$$

(c) Freeman - Tukey's Transformation (FREEMAN MURRAY F.; TUKEY, 1950)

This transformation is based on a sum of two square roots and was applied on (2.11)

$$
\widehat{\rho}_{f t}=\frac{\sqrt{X+1}+\sqrt{X}}{\sqrt{Y+1}+\sqrt{Y}}
$$


Similarly, Freeman-Tukey's transformation can also be applied for the test statistic based on the statistic $W_{0}^{1}=\widehat{\lambda}_{1}-\rho_{0} \widehat{\lambda}_{2}$. The expression of the statistic is deduced as below.

$$
Z_{3}=\frac{\sqrt{X+1}+\sqrt{X}-\rho_{0}[\sqrt{Y+1}+\sqrt{Y}]}{\sqrt{1+\rho_{0}^{2}}}
$$

(d) Rossi-Lampugnani's Transformation (ROSSI G.; LAMPUGNANI, 1950)

Let $W$ be a generic Poisson random variable. Consider the two transformations $Q_{1}=\frac{W-\lambda}{\sqrt{\lambda}} ; Q_{2}=2 \sqrt{W}$. As $Q_{1}$ and $Q_{2}$ are asymptotically standardized normally distributed, Rossi and Lampugnani (ROSSI G.; LAMPUGNANI, 1950) proposed to use the average of these two transformations which results $\frac{W+2 \sqrt{W \lambda}}{2 \sqrt{\lambda}}$. Applying on (2.11), it yields:

$$
\hat{\rho}_{r s}=\frac{X+2 \sqrt{X \lambda_{10}}}{Y+2 \sqrt{Y \lambda_{20}}} \sqrt{\frac{1}{\rho_{0}}}
$$

Due the possibility of the denominator be zero, an adjustment is required by the addition of 1 to the denominator.

$$
\hat{\rho}_{r s}^{*}=\frac{X+2 \sqrt{X \lambda_{10}}}{Y+2 \sqrt{Y \lambda_{20}}+1} \sqrt{\frac{1}{\rho_{0}}}
$$

Replacing $\lambda_{1}$ and $\lambda_{2}$ in (2.38) by their MLE's, a new statistic can be created:

$$
\hat{\rho}_{r s}^{* *}=\frac{3 X}{3 Y+1} \sqrt{\frac{1}{\rho_{0}}}
$$

Similarly, Rossi's transformation can be applied for statistic (2.14). The expression of the statistic is deduced as below.

$$
Z_{4}=\frac{X+2 \sqrt{X \lambda_{10}}-\rho_{0}\left(Y+2 \sqrt{Y \lambda_{20}}\right)}{2 \sqrt{\lambda_{10}+\lambda_{20} \rho_{0}^{2}}}
$$

In case of $\lambda_{1}$ and $\lambda_{2}$ unknown, the statistic can be expressed:

$$
Z_{4}^{*}=\frac{3 X-3 \rho_{0} Y}{2 \sqrt{X+\rho_{0}^{2} Y}}
$$

To avoid division by zero the addition of 1 to the denominator is used and then the statistics is transformed as the following:

$$
Z_{4}^{* *}=\frac{3 W-3 \rho_{0} Z}{2 \sqrt{W+\rho_{0}^{2} Z}+1}
$$


And finally the last normalized transformation. According to Mood et al. (MOOD; GRAYBILL; DUANE, 1974), the mean and variance of rate of two random variables can be approximated as following:

$$
\begin{gathered}
E\left[\frac{X}{Y}\right] \approx \frac{E(X)}{E(Y)}-\frac{1}{E(Y)^{2}} \operatorname{Cov}[X, Y]+\frac{E(X)}{E(Y)^{3}} \operatorname{Var}[Y] \\
\operatorname{Var}\left[\frac{X}{Y}\right] \approx\left(\frac{E(X)}{E(Y)}\right)^{2}\left(\frac{\operatorname{Var}[X]}{E(X)^{2}}+\frac{\operatorname{Var}[Y]}{E(Y)^{2}}-\frac{2 \operatorname{Cov}[X, Y]}{E(X) E(Y)}\right)
\end{gathered}
$$

So as our variables are Poisson's, $E[X], \operatorname{Var}[X], E\left[X^{2}\right]$ and $E\left[X^{3}\right]$ are respectively

$$
\begin{aligned}
E[X] & =\operatorname{Var}[X]=\lambda_{1} \\
E\left[X^{2}\right] & =\lambda_{1}+\lambda_{1}^{2} \\
E\left[X^{3}\right] & =\lambda_{1}+3 \lambda_{1}^{2}+\lambda_{1}^{3}
\end{aligned}
$$

Furthermore, as $\mathrm{X}$ and $\mathrm{Y}$ are independent so $\operatorname{Cov}[X, Y]$ is zero. Therefore, (2.42) and (2.43) can be reexpressed as

$$
\begin{gathered}
E\left[\frac{X}{Y}\right] \approx \frac{\lambda_{10}}{\lambda_{20}}+\frac{\lambda_{10}}{1+3 \lambda_{20}+\lambda_{20}^{2}} \\
\operatorname{Var}\left[\frac{X}{Y}\right] \approx\left(\frac{\lambda_{10}}{\lambda_{20}}\right)^{2}\left(\frac{1}{1+\lambda_{10}}+\frac{1}{1+\lambda_{20}}\right)
\end{gathered}
$$

So the standardized statistic of $\frac{X}{Y}$ is expressed as following.

$$
Z_{5}^{0}=\frac{(X / Y)-\left[\lambda_{10} / \lambda_{20}+\lambda_{10} /\left(1+3 \lambda_{20}+\lambda_{20}^{2}\right)\right]}{\left(\lambda_{10} / \lambda_{20}\right) \sqrt{1 /\left(1+\lambda_{10}\right)+1 /\left(1+\lambda_{20}\right)}}
$$

In order to avoid division by zero in $\frac{X}{Y}$, the addition of one in its denominator was made

$$
Z_{5}=\frac{[X /(Y+1)]-\left[\lambda_{10} / \lambda_{20}+\lambda_{10} /\left(1+3 \lambda_{20}+\lambda_{20}^{2}\right)\right]}{\left(\lambda_{10} / \lambda_{20}\right) \sqrt{1 /\left(1+\lambda_{10}\right)+1 /\left(1+\lambda_{20}\right)}}
$$

The performance of control chart with this statistic $Z_{5}$ is evaluated in this study as well. And again substituting $\lambda_{1}$ and $\lambda_{2}$ in (2.46) by their MLE's it results in:

$$
Z_{5}^{*}=-\frac{\left(1+4 Y+Y^{2}\right) \sqrt{1+X}}{\left(1+3 Y+Y^{2}\right) \sqrt{(1+Y)(X+Y+2)}} .
$$




\section{Methodology}

In order to evaluate the performance of several statistics presented in the previous chapter, an extensive Monte Carlo simulation study is conducted as the determination of the exact distribution of the statistics is quite complicated.

\subsection{Monte Carlo simulation study}

Monte Carlo simulation is a method to obtain numerical results that might be deterministic in principle with repeated sampling. Monte Carlo simulation is widely used in physical and mathematical problems when it is difficult to solve by calculation formula. In this study the performances of control charts are evaluated in term of ARL. However the control limits with fixed $A R L_{0}$ and $A R L_{1}$ of control charts for the ratio of two Poisson rates have not expressed by mathematical formula yet. Because of that, Monte Carlo simulation is adopted for this study. The simulation was coded in R (R Core Team, 2017) and available upon request.

\subsection{Simulation study's conditions}

We consider $A R L_{0}=170$, which means approximately $\alpha=0.0059$ according to the equation (2.6); the in-control ratios $\rho_{0}=0.4,0.7$ with in-control Poisson rates equal to $\left(\lambda_{10}, \lambda_{20}\right)=(4,10),(16,40)$ for $\rho_{0}=0.4$ and $\left(\lambda_{10}, \lambda_{20}\right)=(7,10),(28,40)$ for $\rho=0.7$. The UCLs are set up with this condition by Monte Carlo simulation in the first stage.

To evaluate the performances of control charts, we calculate $A R L_{1}$ with UCL set up in stage 1 . The out of control condition is made by the shifts on the ratio $\rho$ and on the means $\lambda_{1}$ and $\lambda_{2}$. The shifts of $\rho_{0}$ are expressed as $\rho_{1}=\tau \rho_{0}, \rho_{0}=\frac{\lambda_{10}}{\lambda_{20}}$ with $\tau=(1.02,1.05,1.10,1.20,1.30,1.40,1.50)$, and the shifts of means $\lambda_{10}$ and $\lambda_{20}$ are $\lambda_{11}=\delta_{X} \lambda_{10}, \lambda_{21}=\frac{\lambda_{11}}{\rho_{1}}=\frac{\delta_{x}}{\tau} \lambda_{20}$ with $\delta_{X}=(0.8,0.9,1.0,1.1,1.2)$. The parameter $\delta_{x}$ makes the shift of $\lambda_{1}$ as it is shown in (3.2). At the same time, the shift of $\lambda_{2}$ is defined because it depends on $\rho$ and $\lambda_{1}$ (refer (3.3)). So the parameter $\delta_{x}$ makes $\lambda_{1}$ and $\lambda_{2}$ increase (decrease) together under fixed $\rho$.

$$
\begin{gathered}
\rho_{1}=\tau \rho_{0} \text { where } \rho_{0}=\frac{\lambda_{10}}{\lambda_{20}} \\
\lambda_{11}=\delta_{X} \lambda_{10} \\
\lambda_{21}=\frac{\lambda_{11}}{\rho_{1}}
\end{gathered}
$$




\subsection{Simulation flow chart}

Two simulations are established. One is for the stage I which defines UCL with desired $A R L_{0}$ and another is for stage II to get $A R L_{1}$.

The flow chart of simulation for stage I is presented in the Figure 2. Firstly the means $\lambda_{10}$ and $\lambda_{20}$ are defined and then the data sets of random variables $\mathrm{X}$ and $\mathrm{Y}$ are generated with the defined means. As a consequence, the statistics are estimated with the data sets of X and Y. After that, we define the initial UCL and simulate 5,000 in-control run lengths. If the $A R L$ achieves $A R L_{0}$, then stops. Otherwise choose another UCL (by increments of $\epsilon=0.00001$ and repeat until the $A R L_{0}$ of 170 is reached. The $A R L_{0}$ is estimated by 5,000 in-control run lengths.

The flow chart of simulation for stage II is shown in the Figure 3. At first the means $\lambda_{10}, \lambda_{20}$ and UCL, which is fixed in stage I, are set up. Furthermore, the parameters $\tau$ and $\delta_{X}$ are selected. In next step, the data set of $\mathrm{X}$ and $\mathrm{Y}$ are generated with the selected parameters and the data sets of statistics are created. Then 5,000 out-of-control run lengths are simulated and $A R L_{1}$ is calculated. 


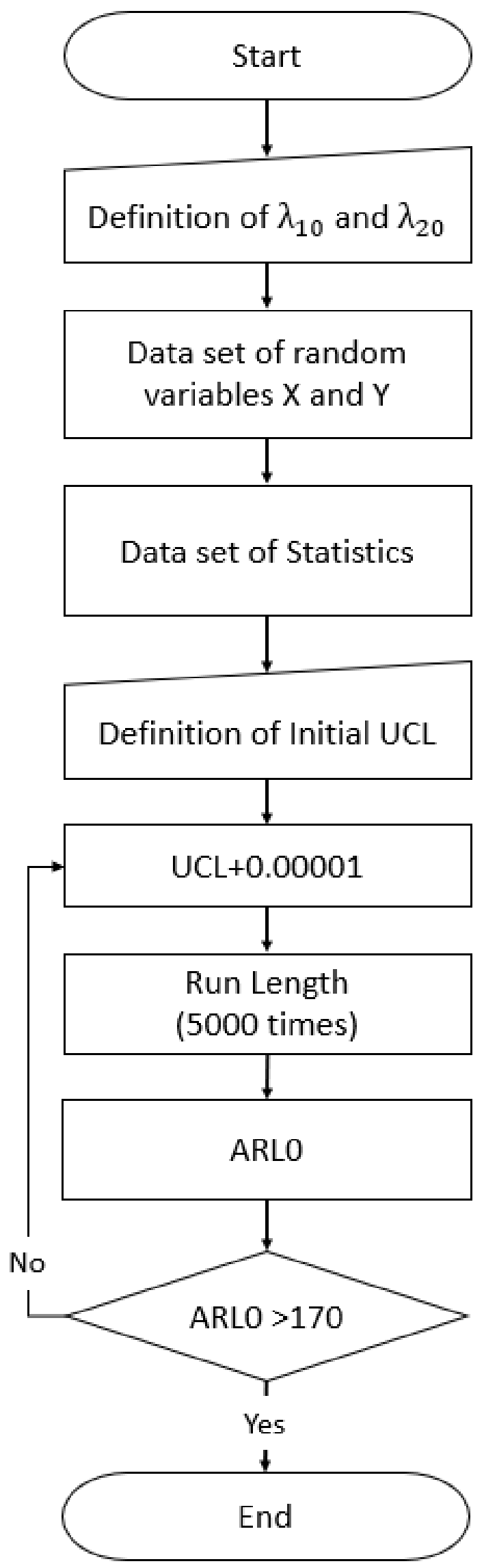

Figure 2 - Simulation Flow Chart for stage I 


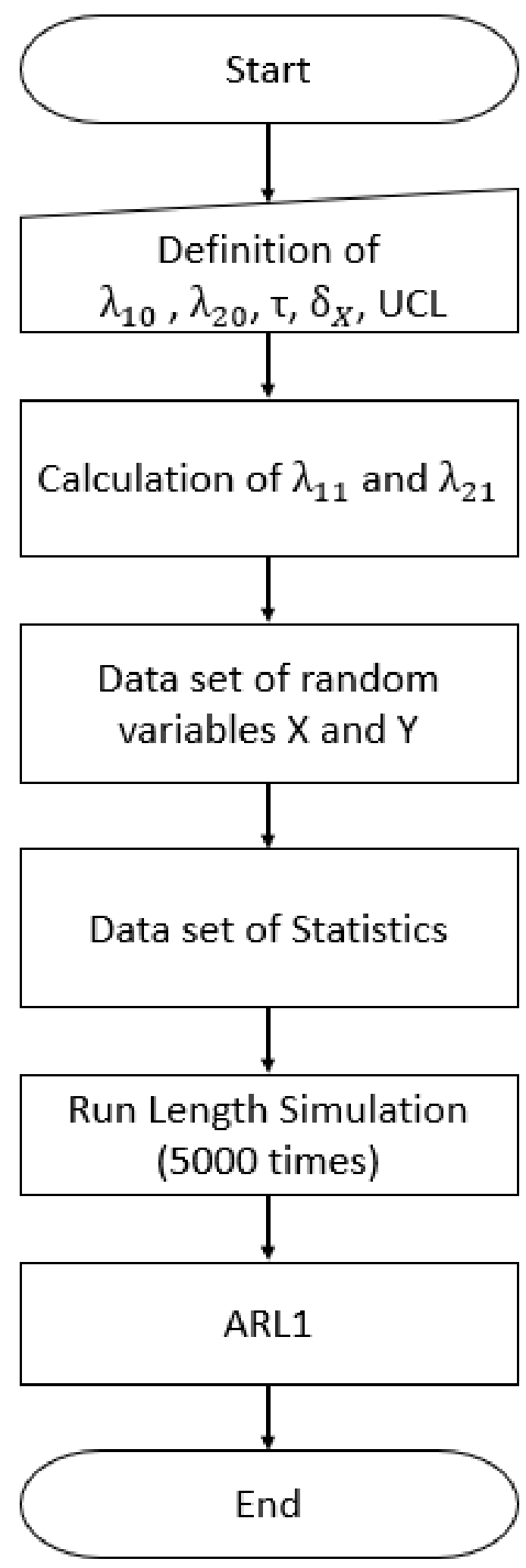

Figure 3 - Simulation Flow Chart for stage II 


\section{Results from simulation study}

In this chapter the results from simulation studies are presented. In Section 4.1 the results with Shewhart control charts are shown and we describe the analysis of them. The results with EWMA type control charts are discussed in Section 4.2. Finally we give an overview of results in Section 4.3. The evaluated statistics are summarized in Table 1.

Table 1 - A summary of the statistics

\begin{tabular}{|c|c|c|c|}
\hline Statistics & Ref. No. & Statistics & Ref. No. \\
\hline$\hat{\rho}_{s d}^{*}=\frac{X}{Y+1}$ & $(2.24)$ & $\hat{\rho}_{s q}^{*}=\frac{\sqrt{X}}{\sqrt{Y+1}}$ & $(2.32)$ \\
\hline$\hat{\rho}_{a s}=\sqrt{\frac{X+\frac{3}{8}}{Y+\frac{3}{8}}}$ & $(2.34)$ & $\hat{\rho}_{f t}=\frac{\sqrt{X+1}+\sqrt{X}}{\sqrt{Y+1}+\sqrt{Y}}$ & $(2.36)$ \\
\hline$\hat{\rho}_{r s}^{*}=\frac{X+2 \sqrt{X \lambda_{10}}}{Y+2 \sqrt{Y \lambda_{20}}+1} \sqrt{\frac{1}{\rho_{0}}}$ & (2.38) & $\hat{\rho}_{r s}^{* *}=\frac{3 X}{3 Y+1} \sqrt{\frac{1}{\rho_{0}}}$ & $(2.39)$ \\
\hline$W_{0}=X-Y$ & $(2.12)$ & $W_{1}=\frac{X-\rho_{0} Y}{\sqrt{\lambda_{1}+\rho_{0}^{2} \lambda_{2}}}$ & $(2.15)$ \\
\hline$W_{1}^{* *}=\frac{X-\rho_{0} Y}{\sqrt{X+\rho_{0}^{2} Y+1}}$ & $(2.26)$ & $W_{2}=\frac{X-\rho_{0} Y}{\sqrt{\left(\lambda_{1}+\lambda_{2}\right) \rho_{0}}}$ & $(2.17)$ \\
\hline$W_{2}^{* *}=\frac{X-\rho_{0} Y}{\sqrt{(X+Y) \rho_{0}+1}}$ & $(2.27)$ & $W_{3}^{\star}=\frac{\ln [(X+1) /(Y+1)]-\ln \left(\rho_{0}\right)}{\sqrt{1 / \lambda_{1}+1 / \lambda_{2}}}$ & $(2.28)$ \\
\hline$W_{3}^{* *}=\frac{\ln [(X+1) /(Y+1)]-\ln \left(\rho_{0}\right)}{\sqrt{1 /(X+1)+1 /(Y+1)}}$ & $(2.29)$ & $W_{4}^{\star}=\frac{\ln [(X+1) /(Y+1)]-\ln \left(\rho_{0}\right)}{\sqrt{\left(2+1 / \rho_{0}+\rho_{0}\right) /\left(\lambda_{1}+\lambda_{2}\right)}}$ & (2.30) \\
\hline$W_{4}^{* *}=\frac{\ln [(X+1) /(Y+1)]-\ln \left(\rho_{0}\right)}{\sqrt{\left(2+1 / \rho_{0}+\rho_{0}\right) /(X+Y+1)}}$ & $(2.31)$ & $Z_{1}=\frac{2\left[\sqrt{X}-\sqrt{\rho_{0} Y}\right]}{\sqrt{1+\rho_{0}}}$ & $(2.33)$ \\
\hline$Z_{2}=\frac{2\left[\sqrt{X+3 / 8}-\sqrt{\rho_{0}(Y+3 / 8)}\right]}{\sqrt{1+\rho_{0}}}$ & $(2.35)$ & $Z_{3}=\frac{\sqrt{X+1}+\sqrt{X}-\rho_{0}[\sqrt{Y+1}+\sqrt{Y}]}{\sqrt{1+\rho_{0}^{2}}}$ & (2.37) \\
\hline$Z_{4}=\frac{X+2 \sqrt{X \lambda_{10}}-\rho_{0}\left(Y+2 \sqrt{Y \lambda_{20}}\right)}{2 \sqrt{\lambda_{10}+\lambda_{20} \rho_{0}^{2}}}$ & $(2.40)$ & $Z_{4}^{* *}=\frac{3 X-3 \rho_{0} Y}{2 \sqrt{X+\rho_{0}^{2} Y}+1}$ & $(2.41)$ \\
\hline$Z_{5}=\frac{[X /(Y+1)]-\left[\lambda_{10} / \lambda_{20}+\lambda_{10} /\left(1+3 \lambda_{20}+\lambda_{20}^{2}\right)\right]}{\left(\lambda_{10} / \lambda_{20}\right) \sqrt{1 /\left(1+\lambda_{10}\right)+1 /\left(1+\lambda_{20}\right)}}$ & $(2.46)$ & $Z_{5}^{*}=-\frac{\left(1+4 Y+Y^{2}\right) \sqrt{1+X}}{\left(1+3 Y+Y^{2}\right) \sqrt{(1+Y)(X+Y+2)}}$ & $(2.47)$ \\
\hline
\end{tabular}




\subsection{Shewhart control chart's performances}

\subsubsection{Case of $\lambda_{10}=7$ and $\lambda_{20}=10$}

Under the same conditions of $\tau$ and $\delta_{X}$, "equal" performance is considered if $A R L_{1}-\min \left(A R L_{1}^{\prime} s\right) \leq 0.5$. Tables $2-7$ present the results related to $\rho_{0}=0.7$ and $A R L_{1}$ values which satisfy such condition are bold. In order to simplify the explanation, we consider that $\tau \leq 1.20$ are low shifts and $\tau>1.20$ are high shifts. And we consider that $\delta_{X} \leq 1.00$ are low ranges and $\delta_{X}>1.00$ are high ranges.

Tables 2 shows the results of the simulation for the Shewhart-type chart about the case of $\lambda_{10}=7$ and $\lambda_{20}=10$.

For larger increases in the average $\left(\delta_{x} \geq 1.10\right)$ and lower shifts in the ratio $(\tau \leq$ 1.10) the statistic $Z_{4}^{* *}$ presents a best performance. For other combinations, in general the statistics $\hat{\rho}_{a s}, \hat{\rho}_{f t}$ and $\hat{\rho}_{r s}^{*}$ can be used as they provides good performance. For a very few combinations of $\tau$ and $\delta_{x}$, the statistics $W_{2}^{*}$ and $W_{4}^{* *}$ can also used employed.

\subsubsection{Case of $\lambda_{10}=28$ and $\lambda_{20}=40$}

Table 3 shows the results for the case $\lambda_{10}=28$ and $\lambda_{20}=40$, the tendency of results is same observed for the case of $\lambda_{10}=7$ and $\lambda_{20}=10$ and they will not repeat here. What differs is for higher shifts in the ratio $(\tau \geq 1.30)$, other statistics besides $\hat{\rho}_{a s}$, $\hat{\rho}_{f t}$ and $\hat{\rho}_{r s}^{*}$ yield "equal" same performance in terms of $A R L_{1}$. It is worth to note that the values of in-control $\lambda_{i 0}, i=1,2$ play an important role in the speed to detect the shifts. A noticeable reduction in $A R L_{1}$ is observed from the former case (smaller in-control rates) to this case.

\subsubsection{Case of $\lambda_{10}=4$ and $\lambda_{20}=10$}

As for the case of $\lambda_{10}=4$ and $\lambda_{20}=10, Z_{3}$ presents the best performance for larger increases in the average $\left(\delta_{x} \geq 1.10\right)$ and lower shifts in the ratio $(\tau \leq 1.10)$ instead of the statistic $Z_{4}^{* *}$ which performs well in case of $\lambda_{10}=7$ and $\lambda_{20}=10$. On the other hand, $\hat{\rho}_{a s}, \widehat{\rho}_{f t}$ and $\hat{\rho}_{a s}$ have good performance for the other combinations, similarly to the case of $\lambda_{10}=7$ and $\lambda_{20}=10 . Z_{1}$ and $Z_{2}$ can be used to complement few combination of $\tau$ and $\delta_{x}$.

\subsubsection{Case of $\lambda_{10}=16$ and $\lambda_{20}=40$}

For larger increases in the average $\left(\delta_{x} \geq 1.10\right)$ and lower shifts in the ratio $(\tau \leq 1.10)$ the statistic $Z_{4}^{* *}$ has the best performances as the cases of $\rho_{0}=0.7$. What differentiate from the other results of Shewhart control chart is that $W_{0}$ became the best 
*

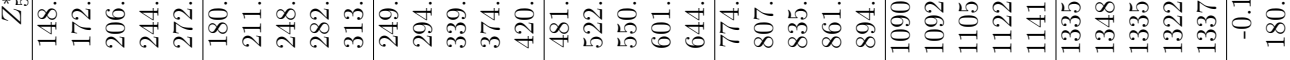

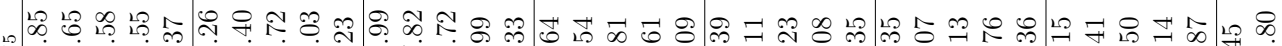

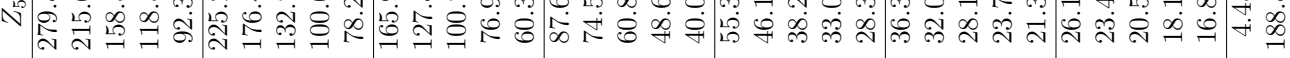

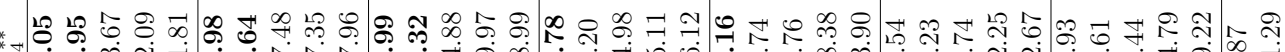

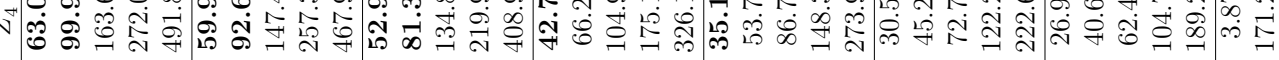

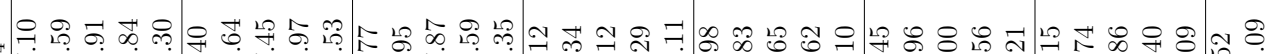

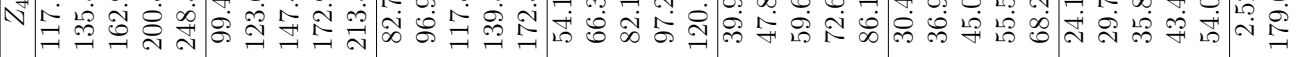

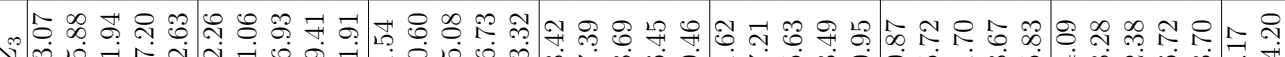

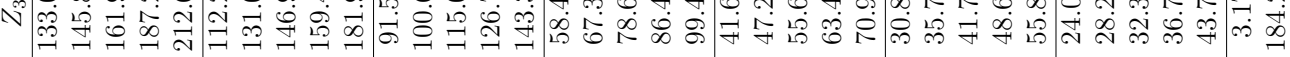

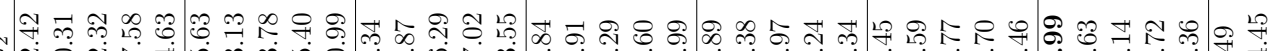

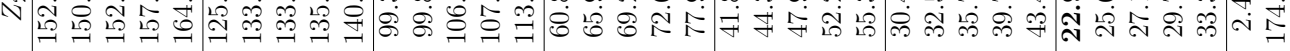

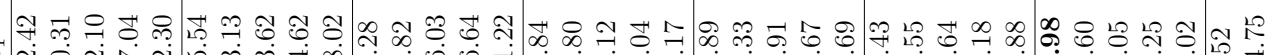

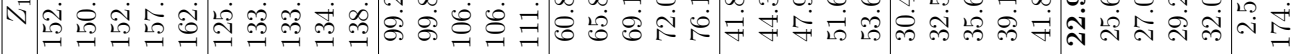

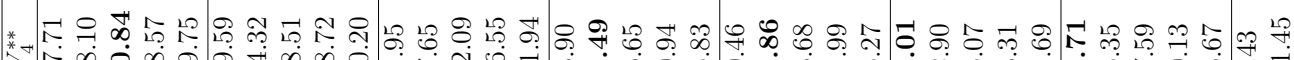

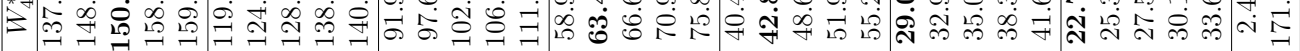

* $=$ 点 *

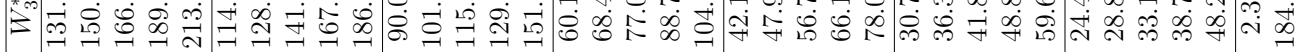

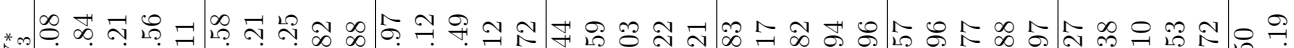

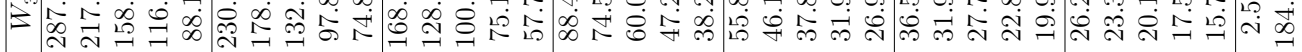
* 둑 웅 = N

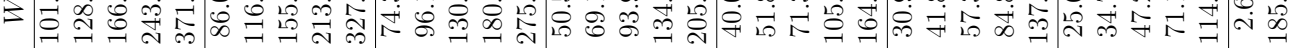
* 2 平 = - 수

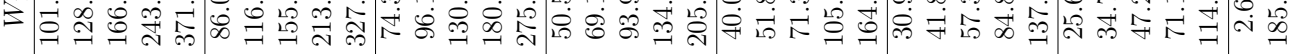

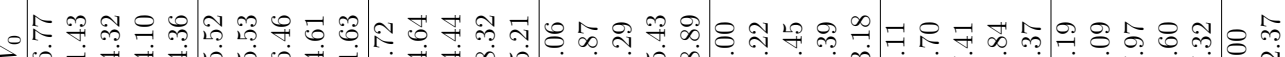

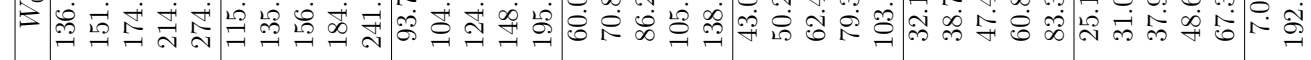

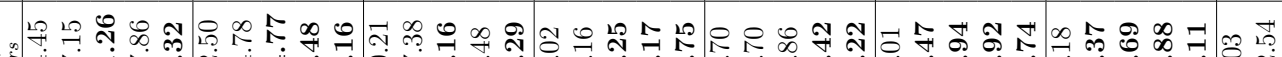

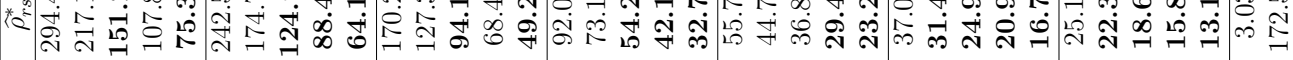

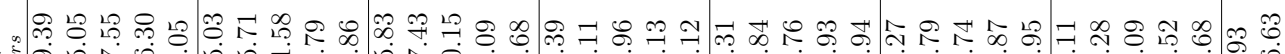

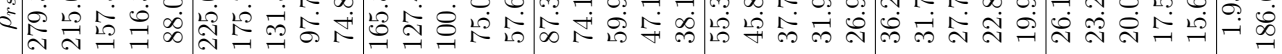

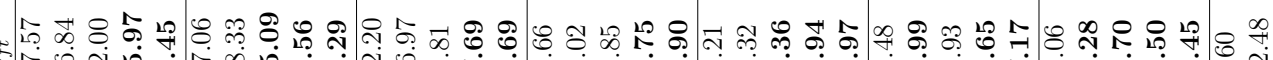

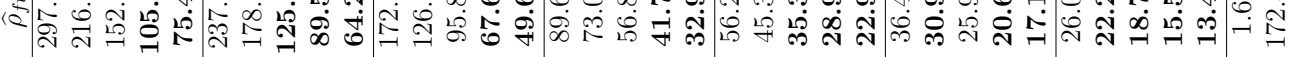

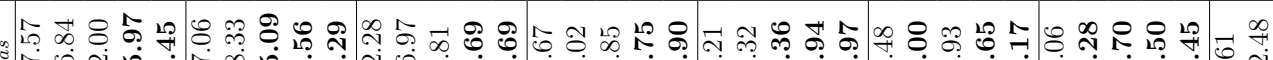

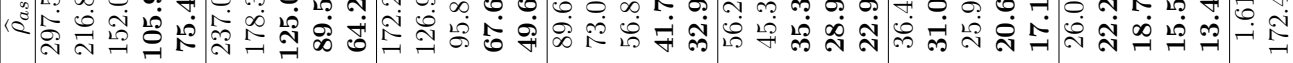

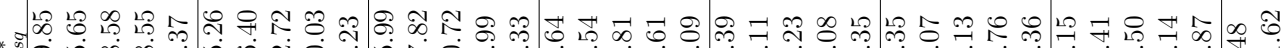
*0.

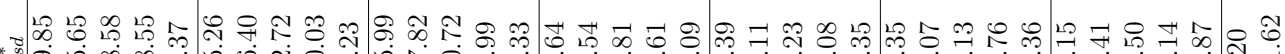
*0.

\begin{tabular}{|c|c|c|c|c|c|c|}
\hline ঋ| & 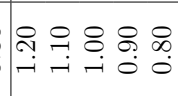 & 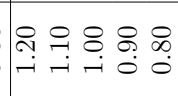 & 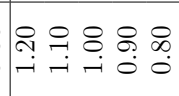 & 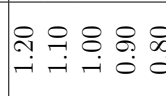 & 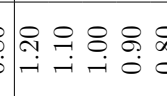 & 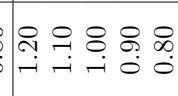 \\
\hline 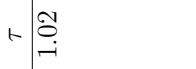 & $\underset{\substack{20 \\
-}}{-i}$ & $\stackrel{0}{\rightleftharpoons}$ & $\stackrel{\text { }}{-}$ & 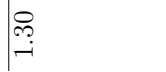 & $\stackrel{ }{\stackrel{P}{\longrightarrow}}$ & 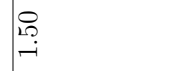 \\
\hline
\end{tabular}




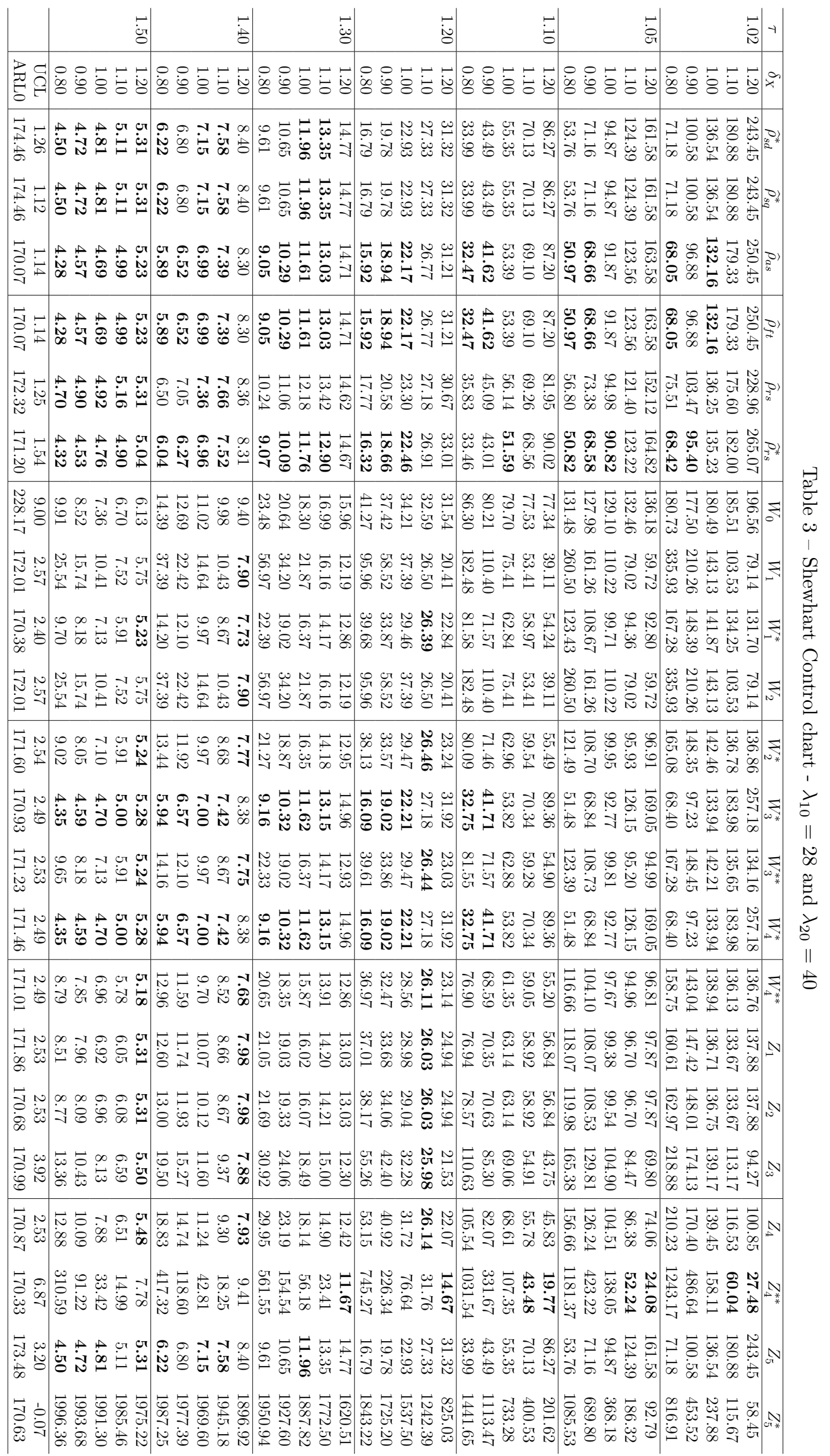


* N

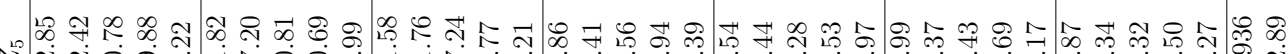

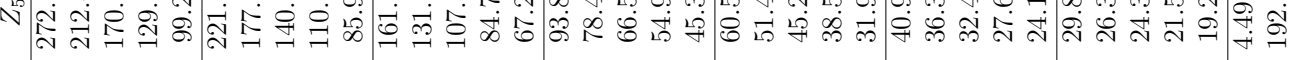

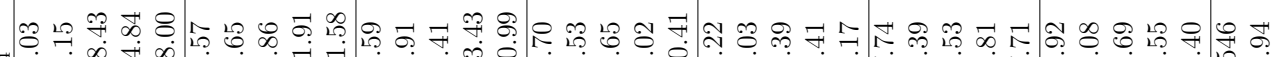

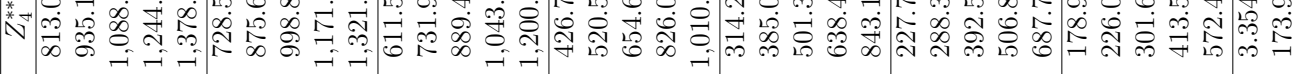

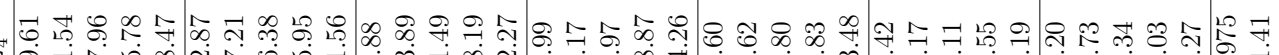

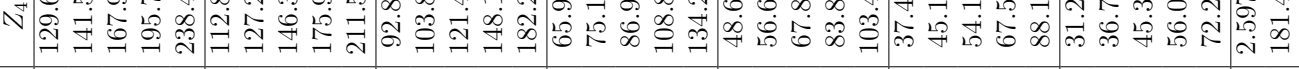

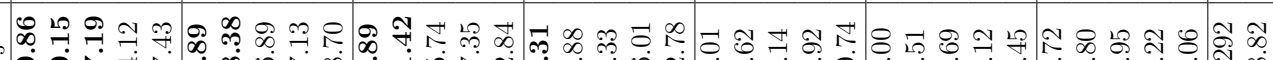

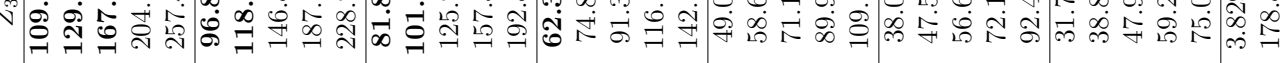

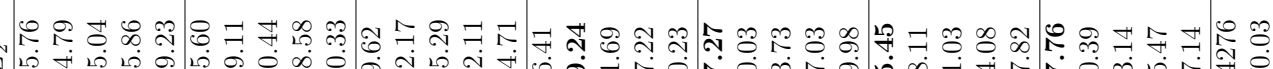

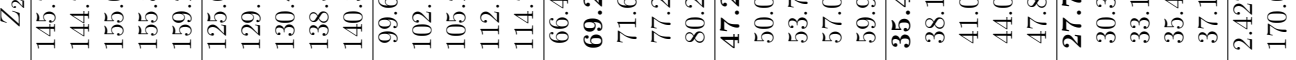

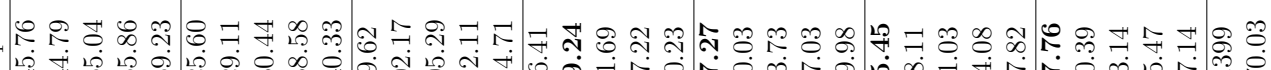

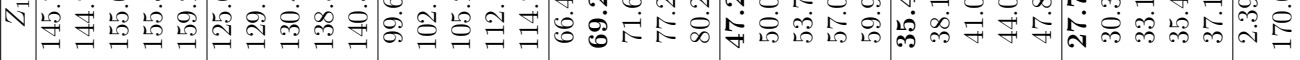

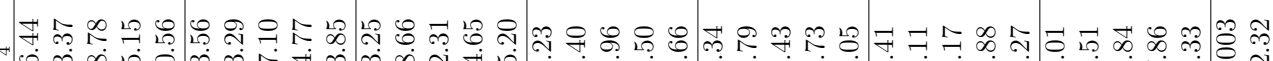

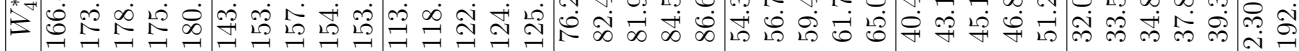

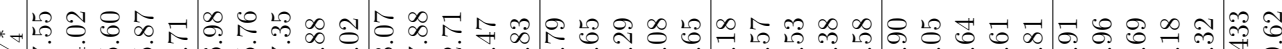

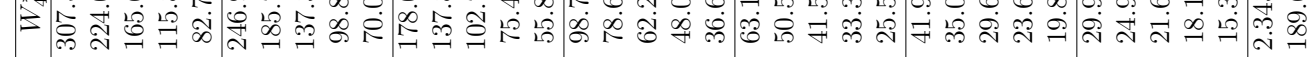

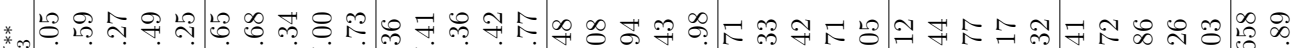

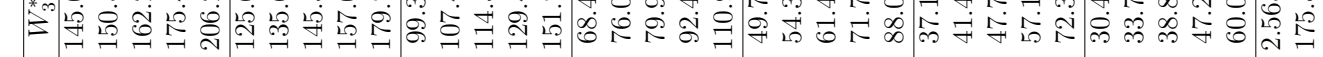
等

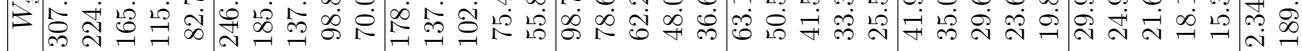

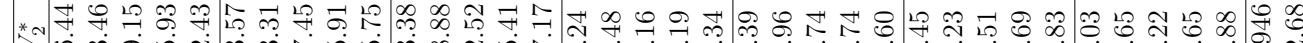

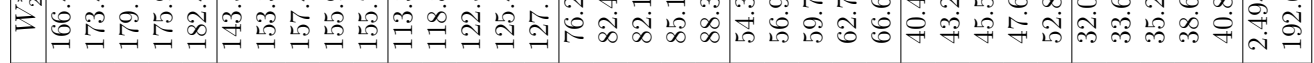

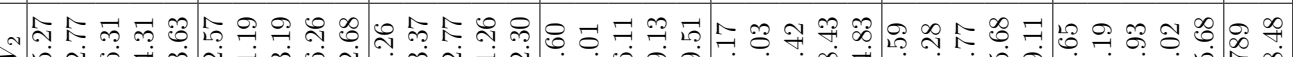

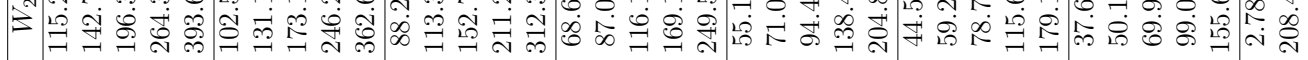
* 웅 占

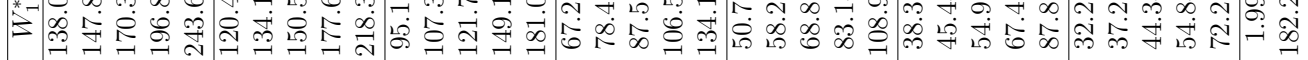

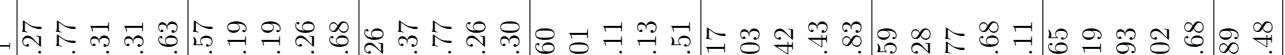

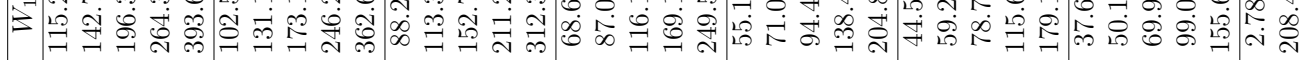

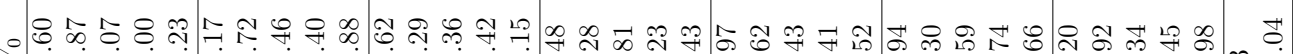

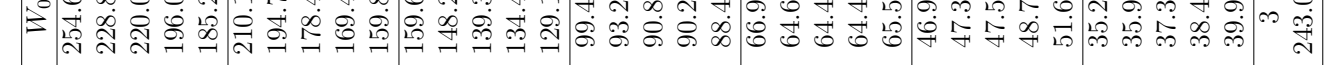

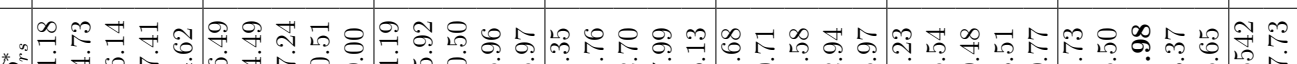
*

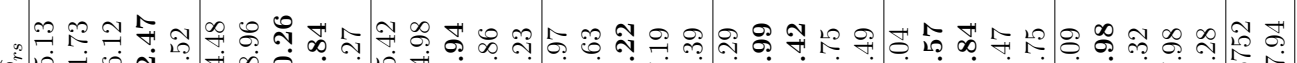

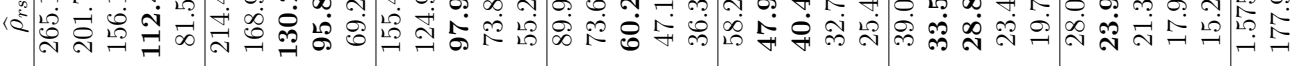
舟

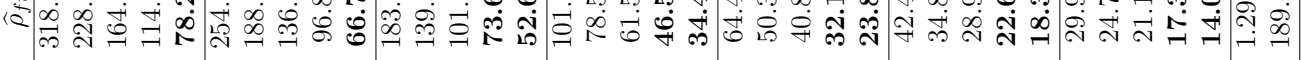
\%

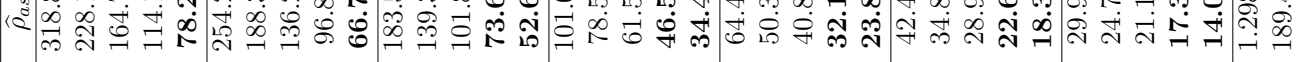

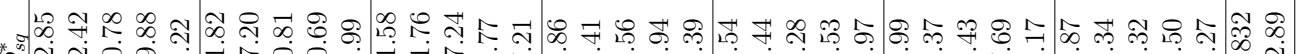

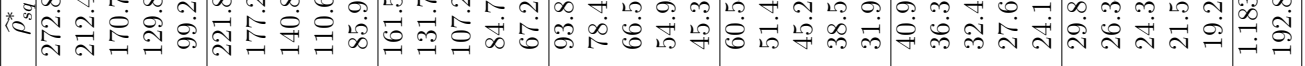

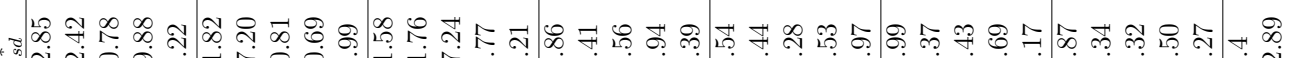

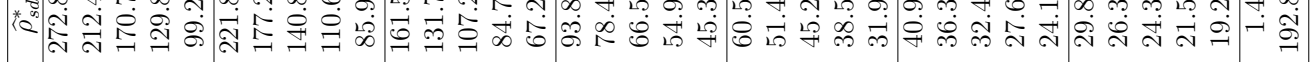

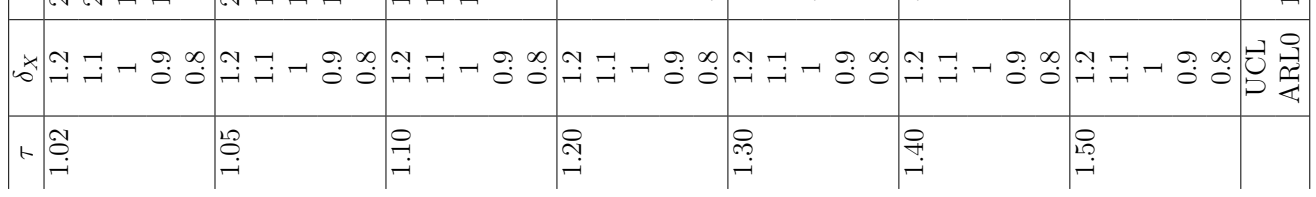


statistic in general except previous combination of $\tau$ and $\delta_{x} . W_{1}^{*}, W_{2}^{*}, W_{3}^{*}$ and $W_{4}^{*}$ present the best performance only for the combination of $\tau=1.30$ and $\delta_{x}=1.2$.

\subsection{EWMA control chart's performance}

EWMA control chart performances are obtained with the constant $\theta=0.2$ and the same previous conditions are considered. Tables 6,7 show the results.

\subsubsection{Case of $\lambda_{10}=7$ and $\lambda_{20}=10$}

For the case $\lambda_{10}=7$ and $\lambda_{20}=10$, the statistic $Z_{4}^{* *}$ also presents good performance for large shift $\delta_{x}=1.20$ and $\tau \leq 1.30$ and when $\delta_{x}=1.10$ and $\tau \leq 1.05$. For other combinations of $\tau$ and $\delta_{x}$, the statistic $W_{4}$ is the best options as it presents the lowest $A R L_{1}$ in most of combinations.

\subsubsection{Case of $\lambda_{10}=28$ and $\lambda_{20}=40$}

For the case $\lambda_{10}=28$ and $\lambda_{20}=40$, the performance of the statistic $Z_{4}^{* *}$ follows closely as the previous case and whenever the statistic $Z_{4}^{* *}$ does not perform well, the $W_{0}$ is the statistic with a better performance. This behavior is observed for $\tau \leq 1.20$. For $\tau \geq 1.30$, several statistics yield similar performance.

\subsubsection{Case of $\lambda_{10}=4$ and $\lambda_{20}=10$}

Tendency is the same as previous cases. $Z_{4}^{* *}$ has the best performances for larger increases in the average and lower shifts in the ratio and $W_{0}$ is the best statistic in general.

\subsubsection{Case of $\lambda_{10}=16$ and $\lambda_{20}=40$}

We could observed also that the case have same character of the previous case as $Z_{4}^{* *}$ and $W_{0}$ cover almost every combination of $\tau$ and $\delta_{x}$. However for $\tau \geq 1.30$, several statistics yield similar performance to $W_{0}$ as we could observed the results of case of $\lambda_{10}=28$ and $\lambda_{20}=40$.

\subsection{Overview of results}

We have "three classes" of statistics: $\rho_{(.)}, W_{(.)}$and $Z_{(.)}$. Some general features are observed: as the shift of the ratio increases $(\tau \uparrow), A R L_{1}$ decreases as expected. As $\delta_{X}$ increases, $A R L_{1}$ may decrease or increase depending on the nature of the statistics. For the statistics of the type $\rho_{(.)}$, as $\delta_{X} \uparrow, A R L_{1} \uparrow$. For the type $W_{(.)}$, different behaviors are observed: as $\delta_{X} \uparrow, A R L_{1} \downarrow$ for $W_{1}$ and $W_{2}$; but for $W_{0}, W_{1}^{*}, W_{2}^{*}, W_{3}^{*}$ and $W_{4}^{*}$, the 
*

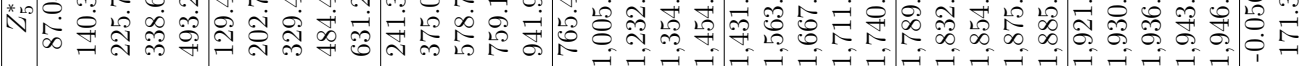

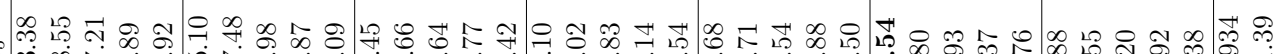

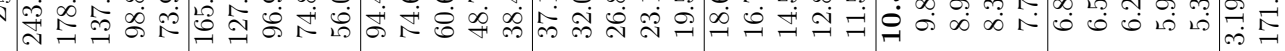

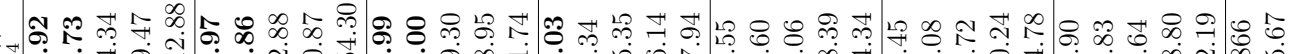

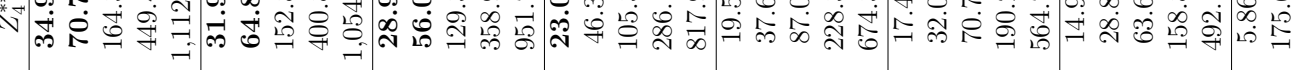

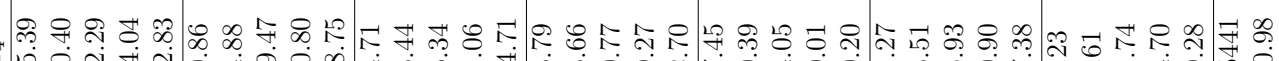

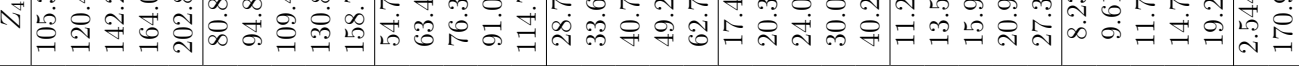

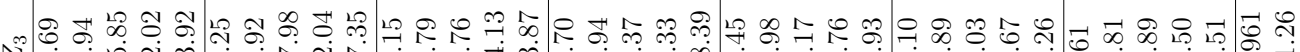

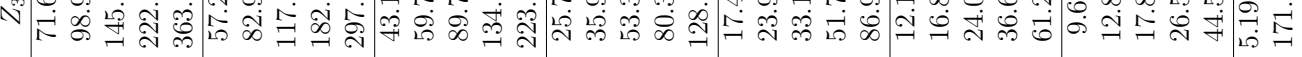

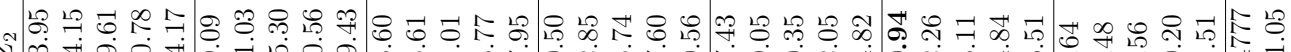

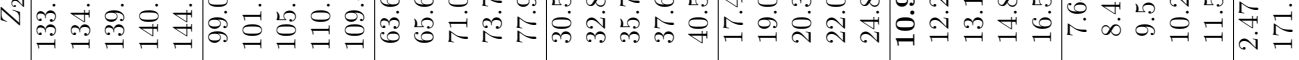

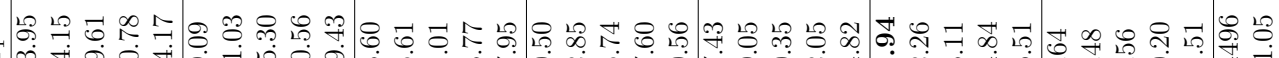

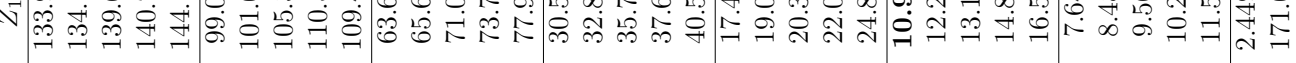

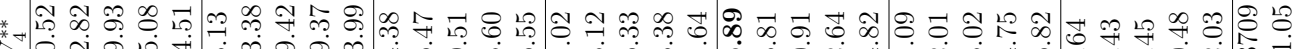

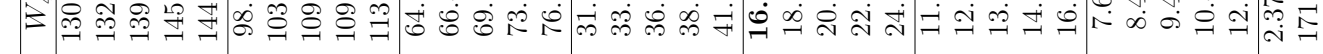

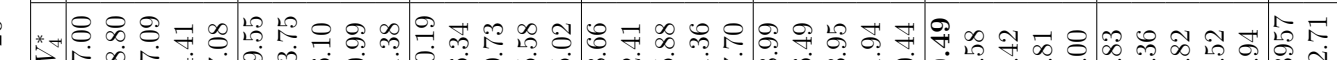

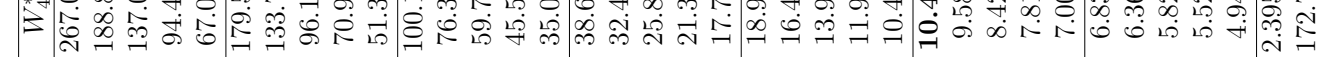

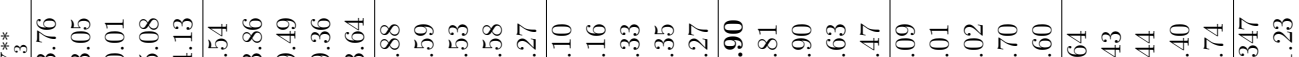

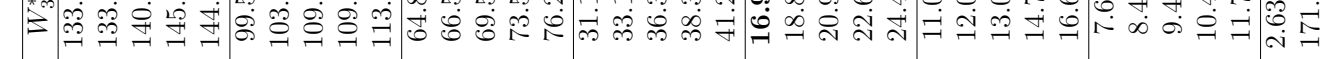

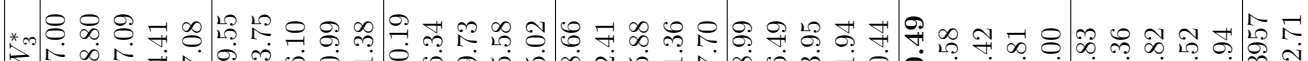

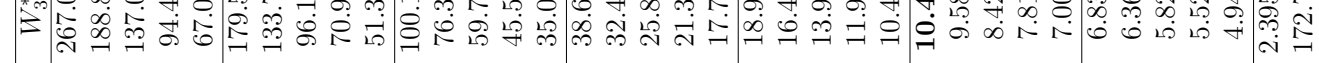
*

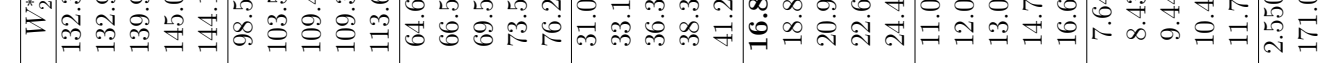

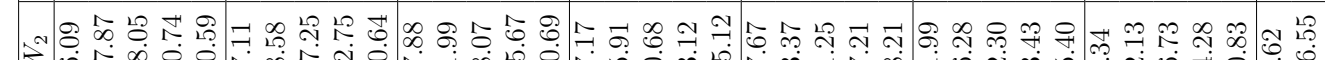

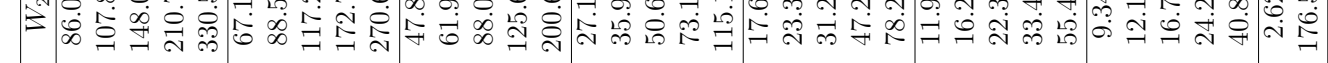

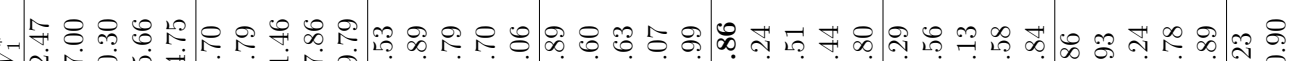

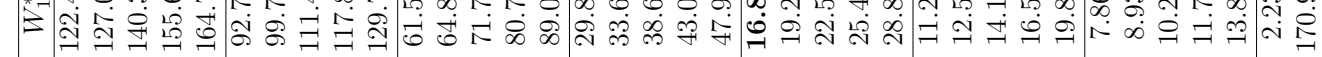

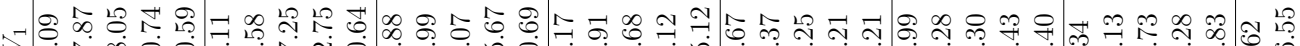

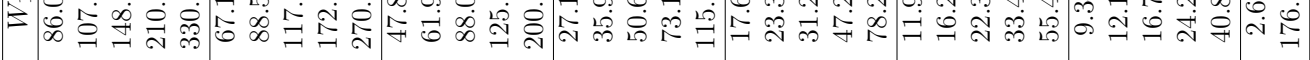

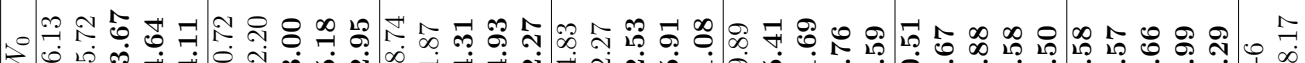

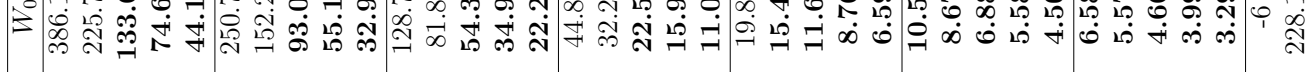

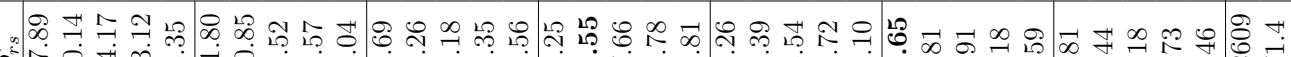

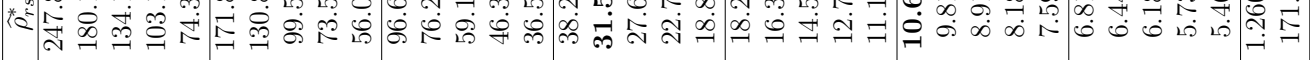

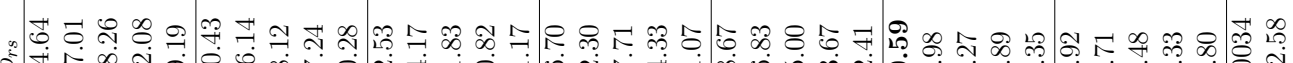

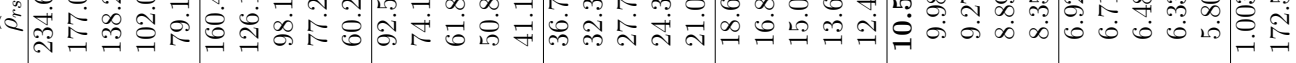

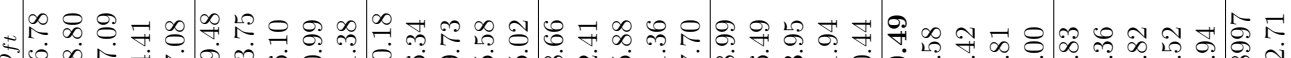

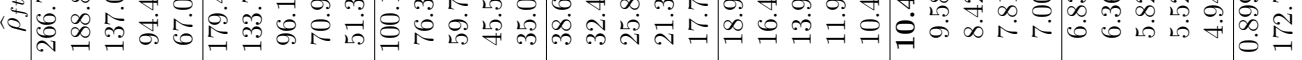
舟

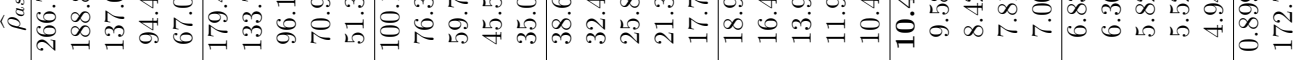
舟识

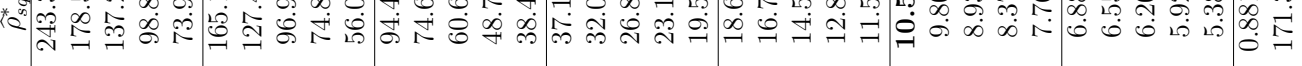

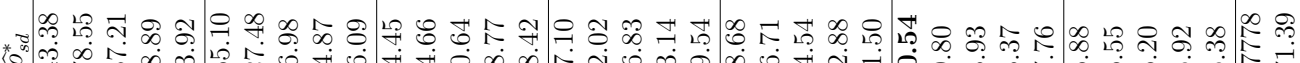
*0.

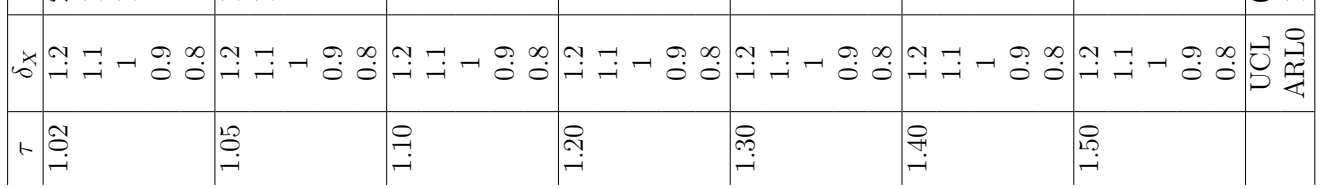




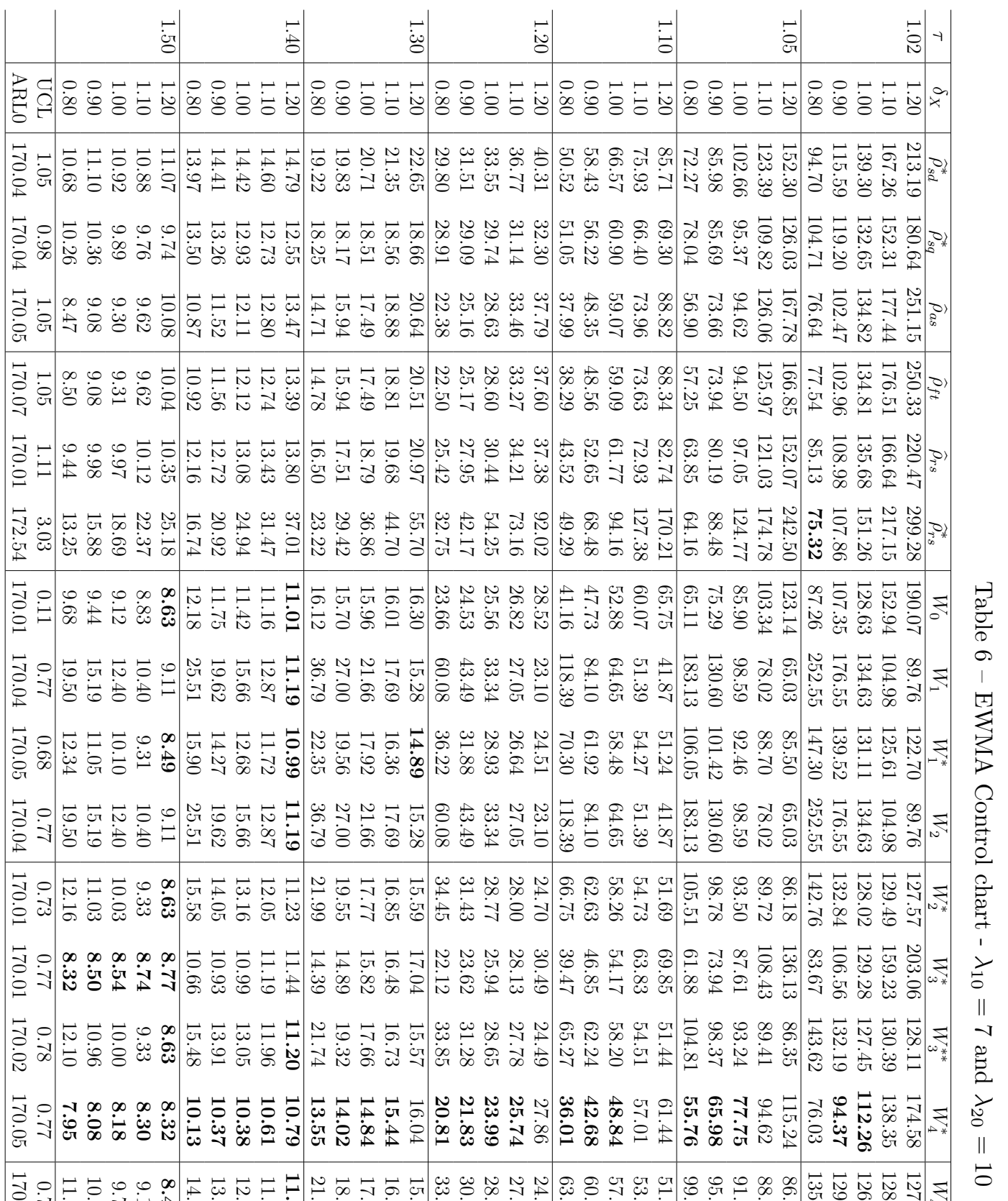

至

겅위 记

ㄱㅇㅇㅂㅜ

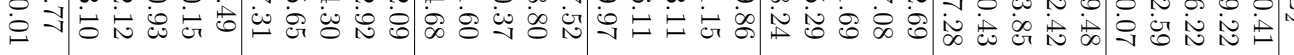

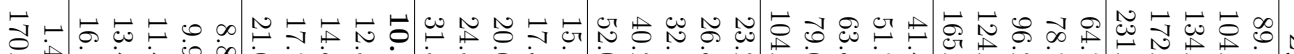

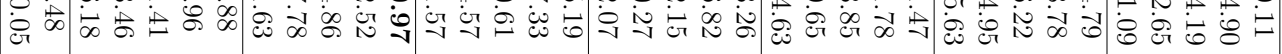

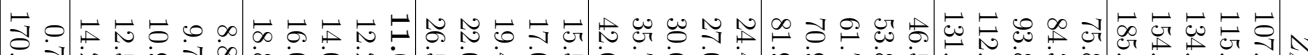

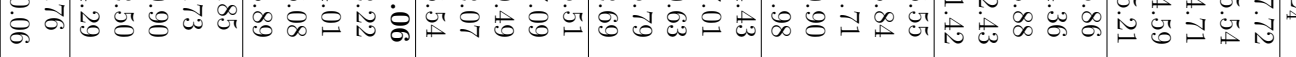
VN

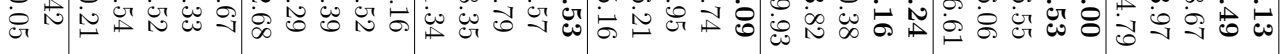
Эo

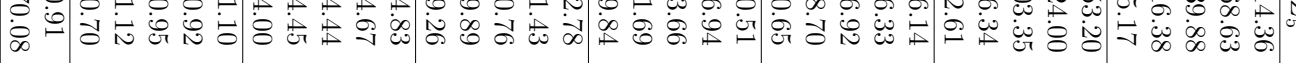

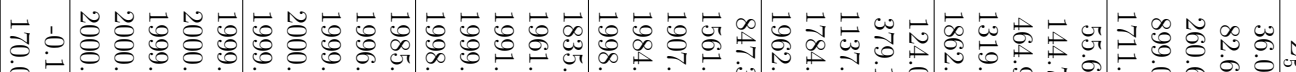

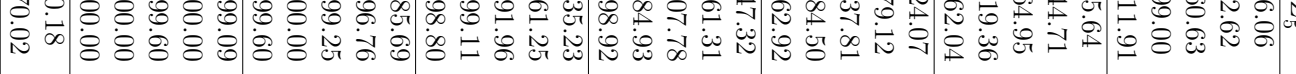


*

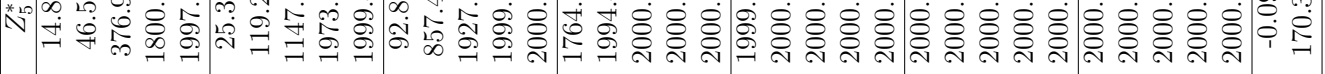

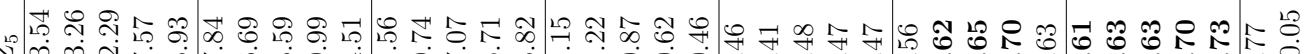

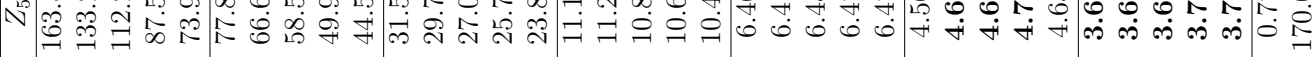

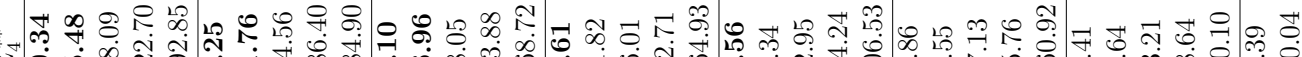
N| 군

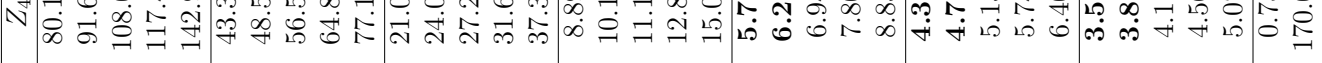
숭

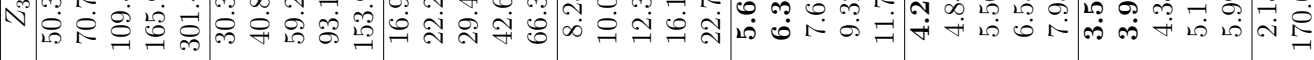

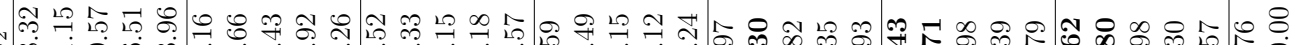

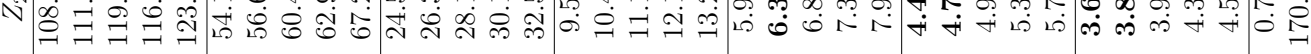

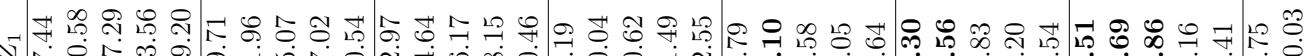

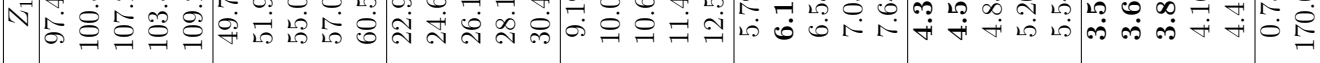

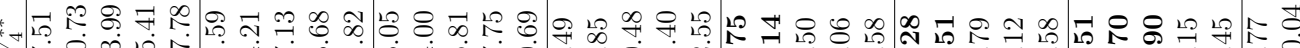

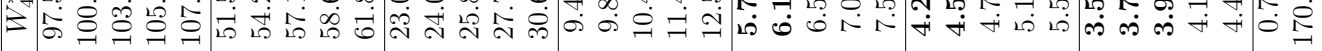

* 숭 ๙

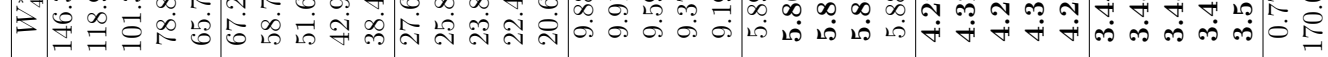
*

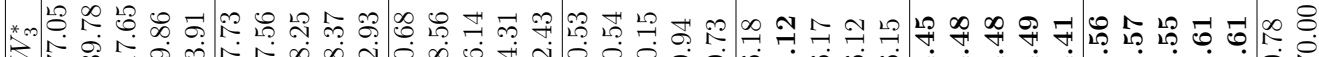

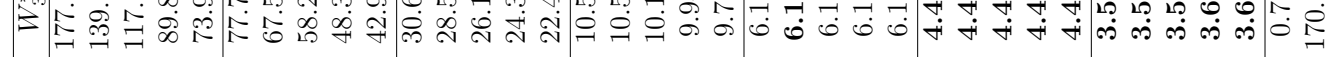
*

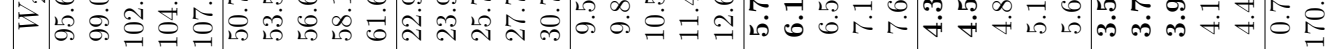

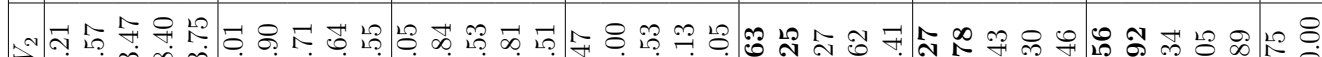

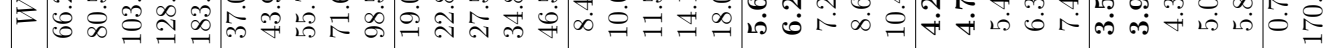

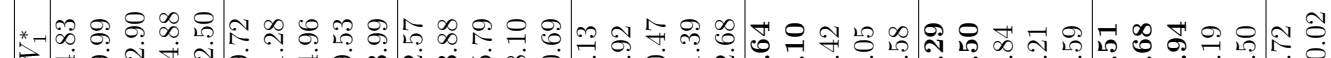

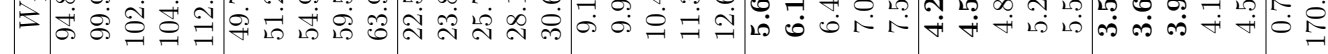

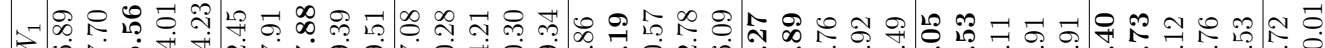
$=$ ف

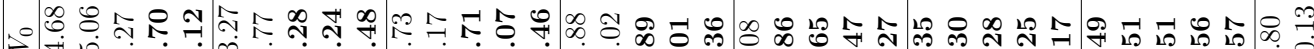

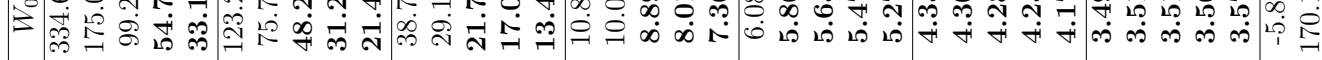

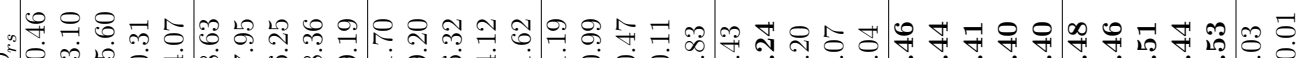
*

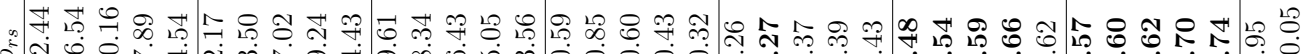

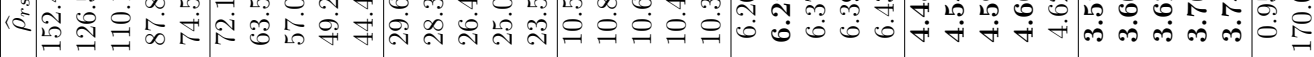

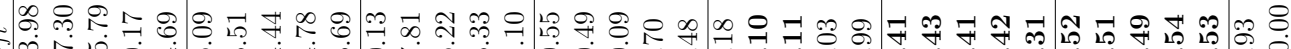

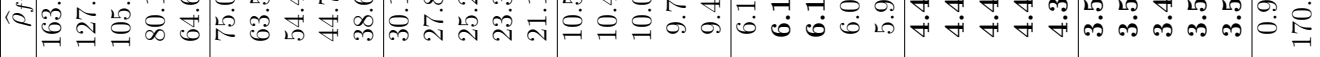

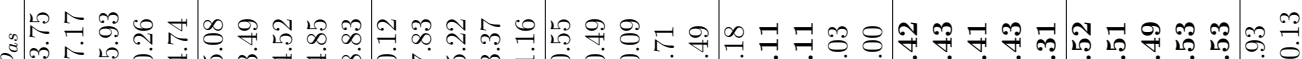

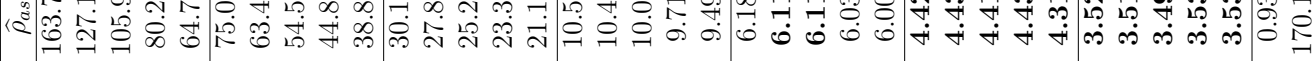
구 \&̊요 *0. ‡ 숭 ำ

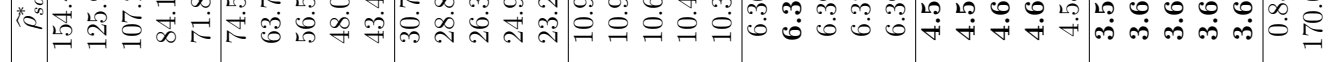

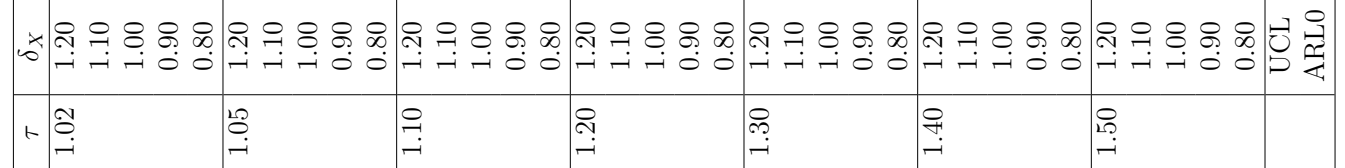




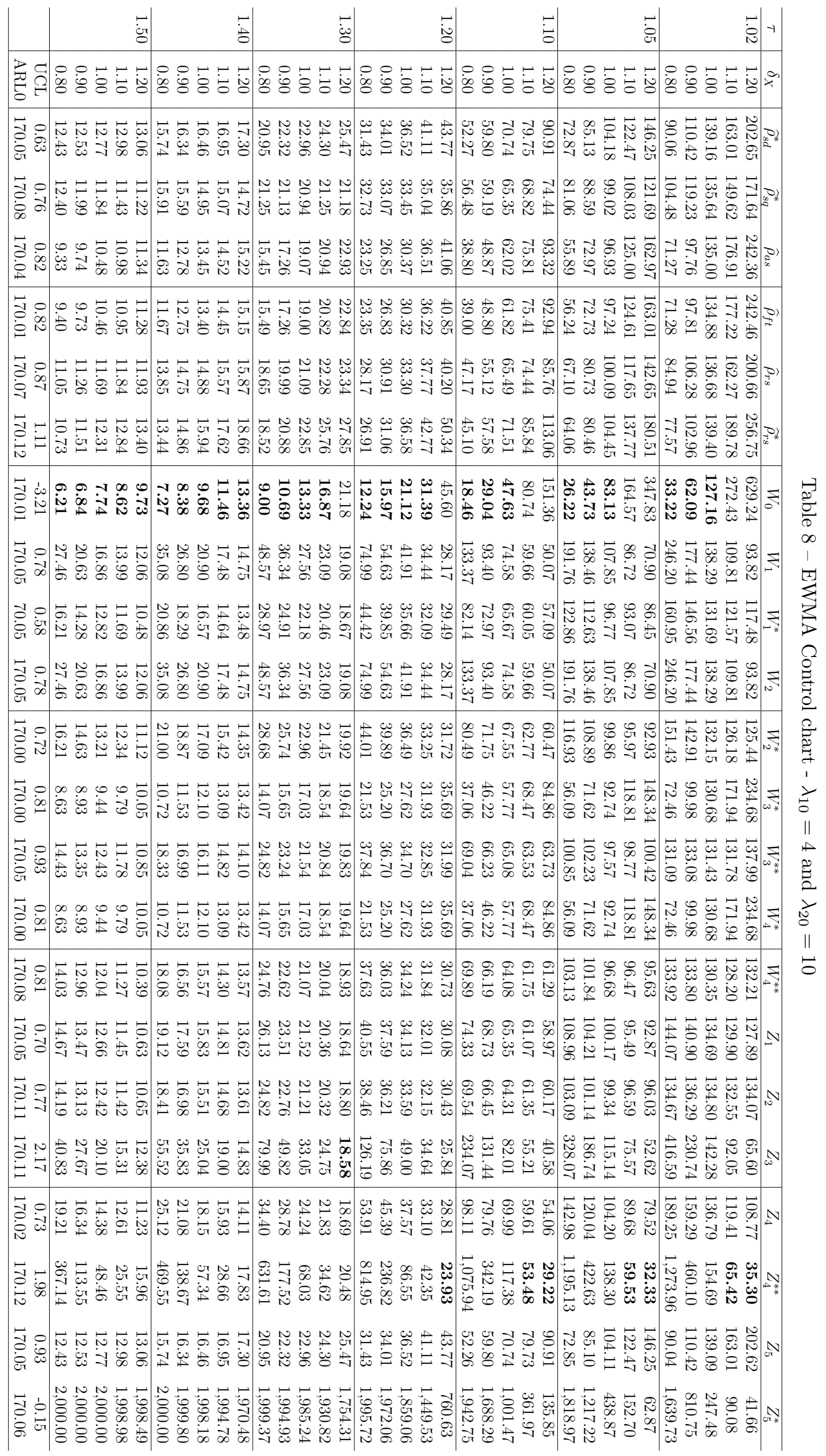


* ผ

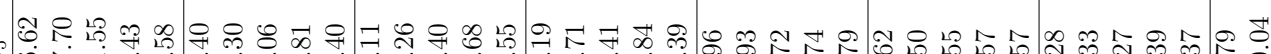

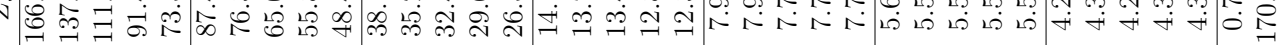

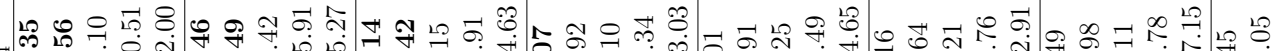

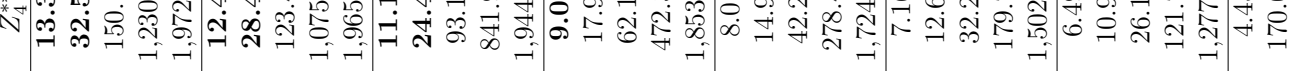

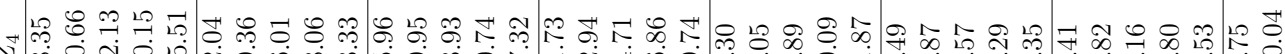

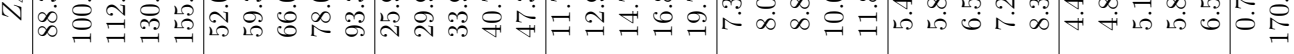

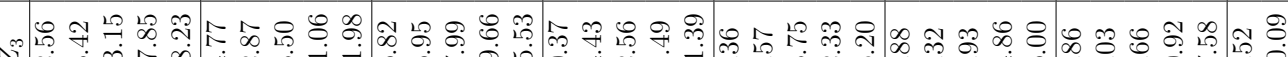

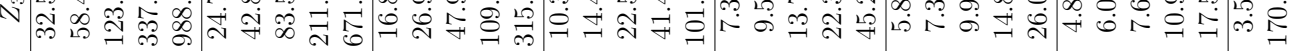

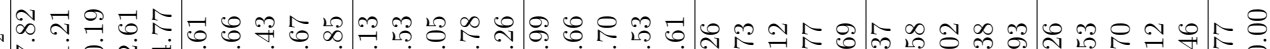

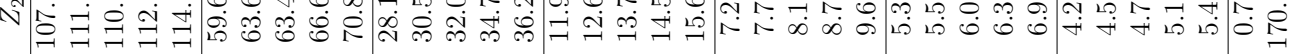

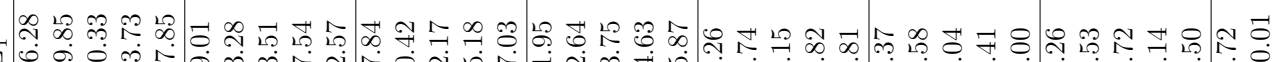

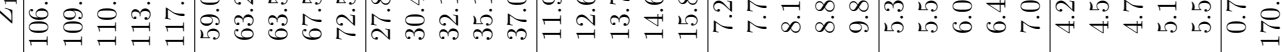
*

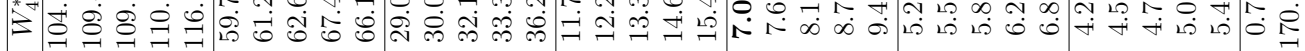

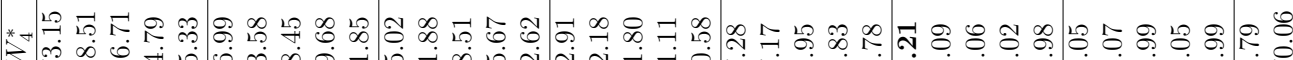
=

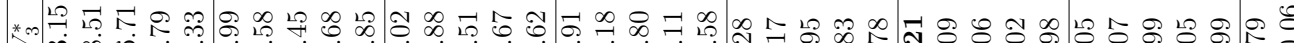

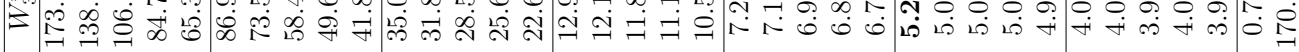

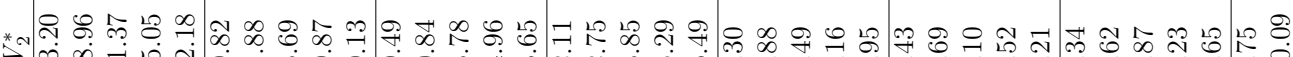

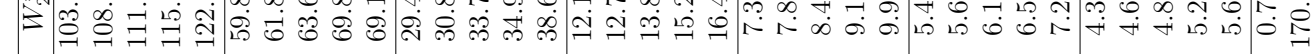

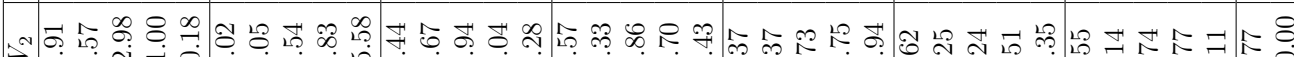

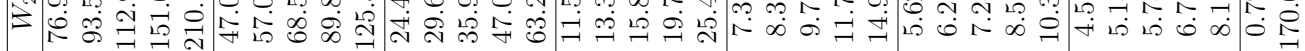

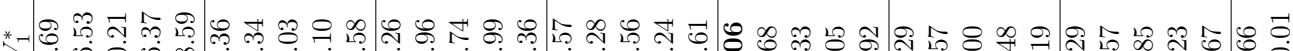

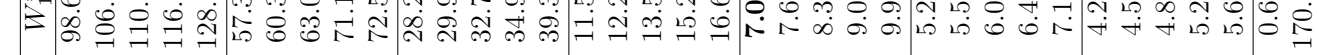

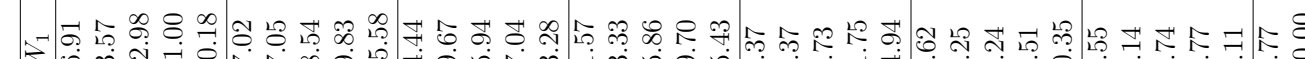

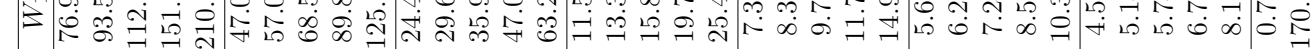

舟 等

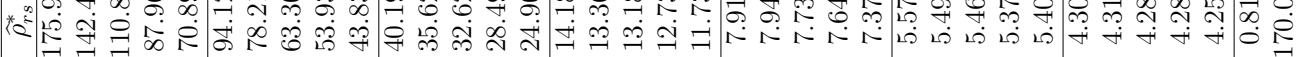

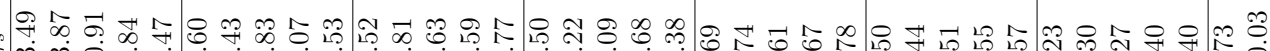

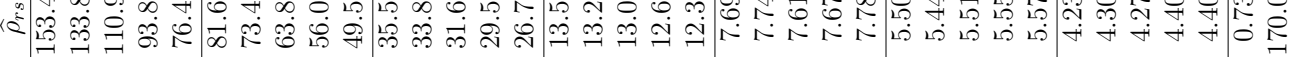
$=8$ 웅 웅

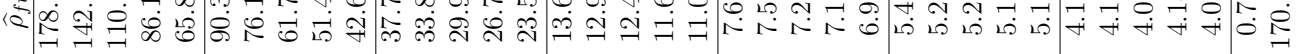

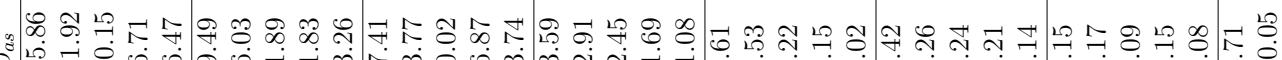

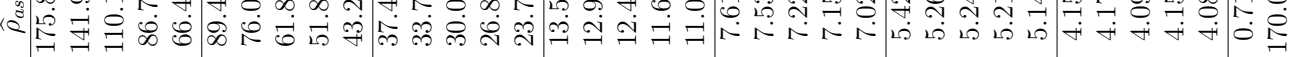

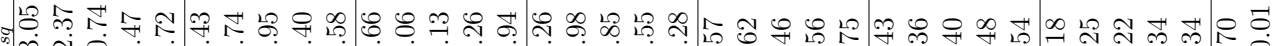

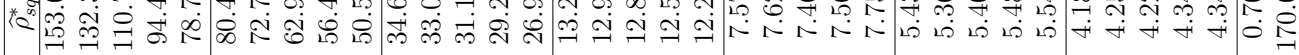

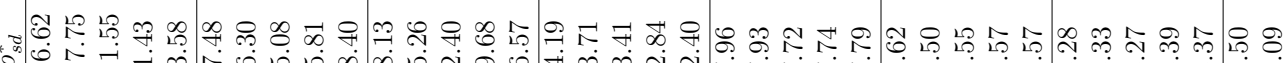

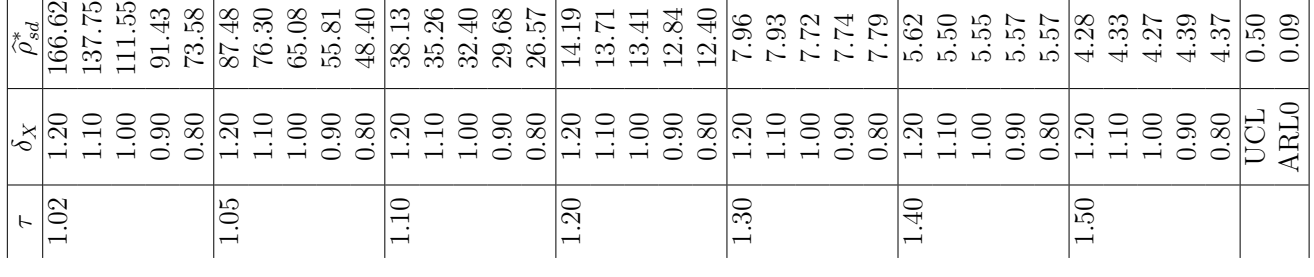


decreases of $A R L_{1}$ are very slowly seeming that they are insensible for $\delta_{X}$ size. For the statistics of type $Z_{(\text {.) }}$, we have the class of insensible as $Z_{1}, Z_{2}, Z_{3}, Z_{4}$; the statistic $Z_{5}$ follows the behavior of the class $\rho_{(.)}$; the statistics $Z_{4}^{* *}$ and $Z_{5}^{*}$ behave like $W_{1}$ and $W_{2}$.

As expected EWMA charts provide better results than Shewhart chart. The values of in-control ratio influence strongly the speed for detection as cases with $\rho_{0}=0.4$ have worse performance than $\rho_{0}=0.7$ mainly in Shewhart charts and $\tau<1.20$. Such influence is weaker in EWMA charts and $\tau \geq 1.20$. Similarly the in-control Poisson rates also affect the speed for detection. The condition $\left(\lambda_{10}, \lambda_{20}\right)=(28,40)$ yield better performance than $\left(\lambda_{10}, \lambda_{20}\right)=(7,10)$ in both charts (coherently the condition $\left(\lambda_{10}, \lambda_{20}\right)=(16,40)$ outperforms than $\left(\lambda_{10}, \lambda_{20}\right)=(4,10)$ for $\left.\rho_{0}=0.4\right)$.

All the simulated cases have as in-control ratios $\rho_{0}=0.4,0.7$. The in-control average Poisson rates as also the in-control ratio influence strongly the speed of detection in both charts mainly for small shifts in the ratio (that is, low values of $\tau$ ) as lower incontrol average rates provide larger $A R L_{1}$. Such effect becomes weaker for larger shifts in the ratio.

Tables 10-11 indicate the statistics which yield the best performance for every combination of shifts $\delta_{x}$ and $\tau$ condition as also the minimum value of $A R L_{1}$ from the simulation study respectively for $\rho_{0}=0.4$ and $\rho_{0}=0.7$.

Some interesting results from Table 10. For example the statistic $Z_{4}^{* *}$ also outperforms for $\delta_{x} \geq 1.10$ and $\tau \leq 1.10$ in EWMA control chart. For other combinations the statistic $W_{0}$ is the best option. These outcomes are also watched for Shewhart chart with in-control Poisson rates $\lambda_{10}=16$ and $\lambda_{20}=40$. Concerning on Shewhart chart for in-control Poisson rates $\lambda_{10}=4$ and $\lambda_{20}=10$, the statistics $\hat{\rho}_{a s}$ and $\hat{\rho}_{f t}$ exhibit good performance for $\delta_{x} \leq 0.90$. For other values of $\delta_{x}$, the statistics like $Z_{1}$ and $Z_{2}$ for $\tau>1.20$ otherwise $Z_{3}$ may be chosen.

From Tables 10-11, we regard that for both types of control charts in case of small shifts on the ratio $(\tau \leq 1.20)$, the shift size on the average rate $\left(\delta_{x}=(1+d) \lambda_{10}\right)$ influences the speed of detection as minimum of $A R L_{1}$ decreases as $|d|$ increases. But for larger shifts $(\tau>1.20)$, this influence is weaker as minimum of $A R L_{1}$ are similar whatever are the values of $\delta_{x}$ mainly for EWMA charts. Additionally when the difference between $\lambda_{10}$ and $\lambda_{20}$ is large like $\lambda_{10}=28$ and $\lambda_{20}=40$ or $\lambda_{10}=16$ and $\lambda_{20}=40$, the best performance relies on the statistics $Z_{4}^{* *}$ and $W_{0}$. 


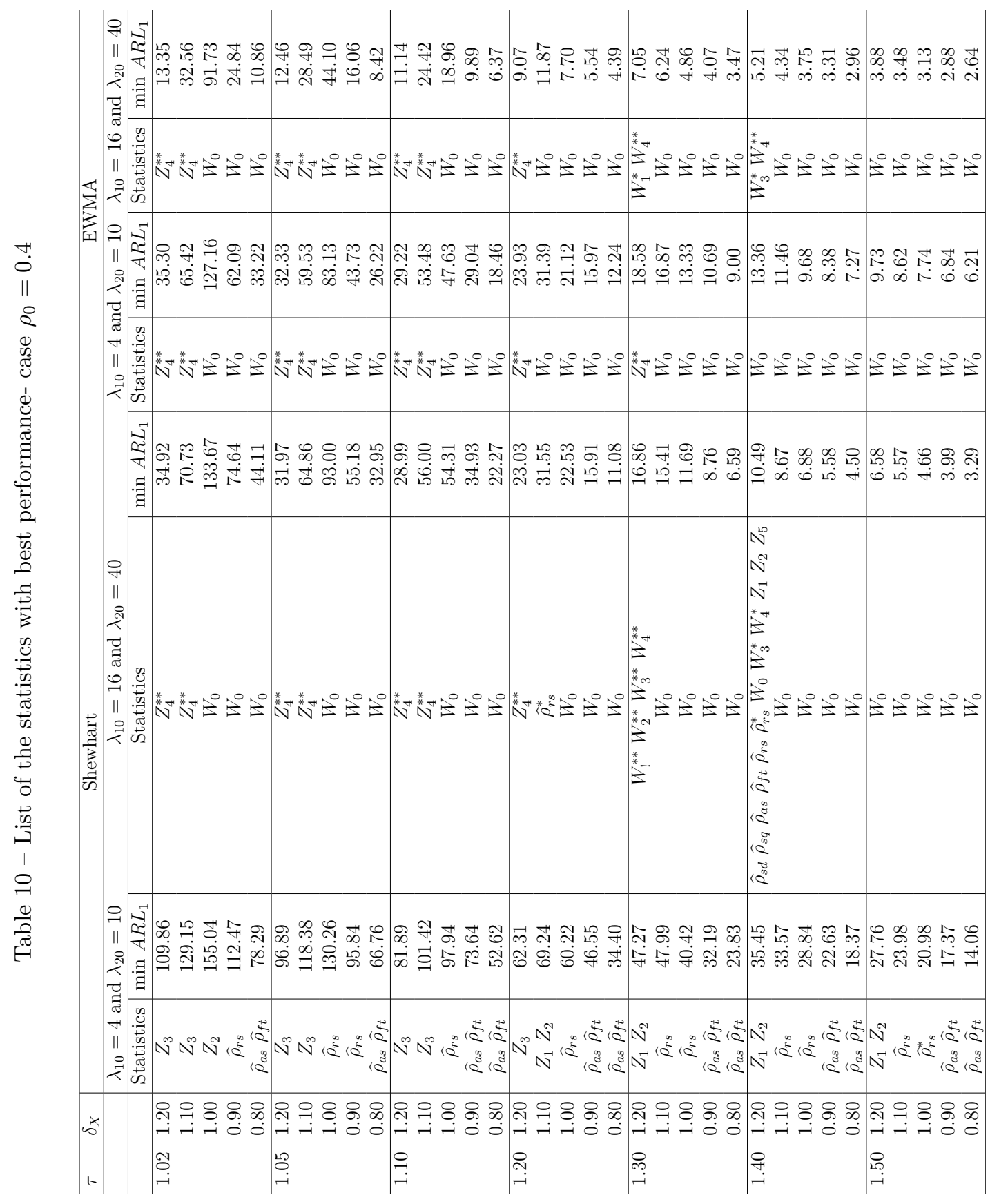




\begin{tabular}{|c|c|c|c|c|c|c|c|c|}
\hline 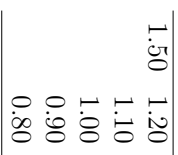 & 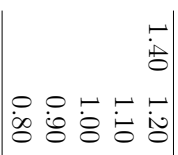 & 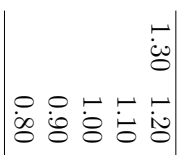 & 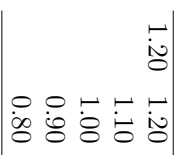 & 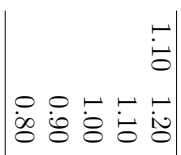 & 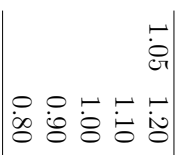 & 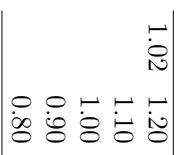 & & \\
\hline 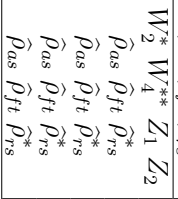 & 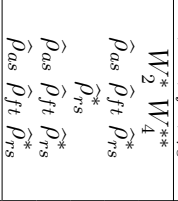 & 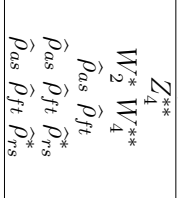 & 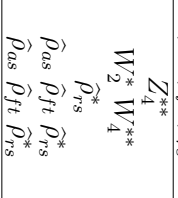 & 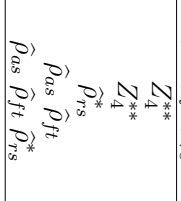 & 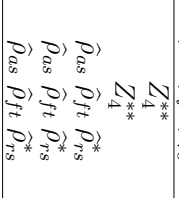 & 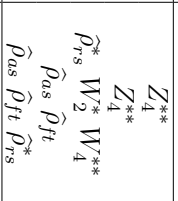 & 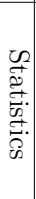 & \\
\hline 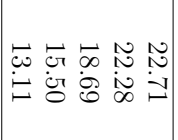 & 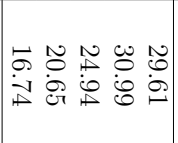 & 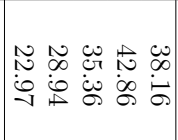 & 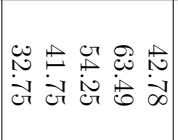 & 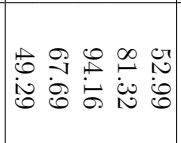 & 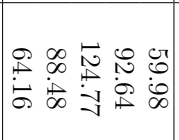 & 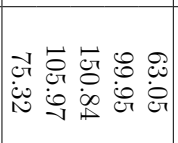 & 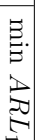 & \\
\hline 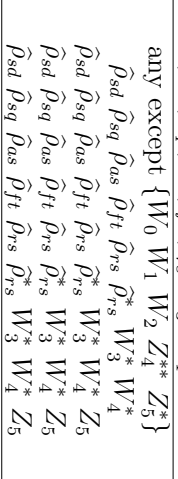 & 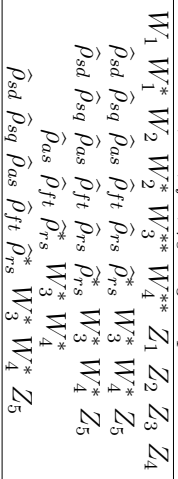 & 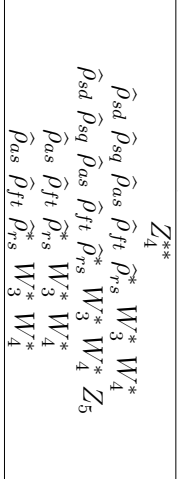 & 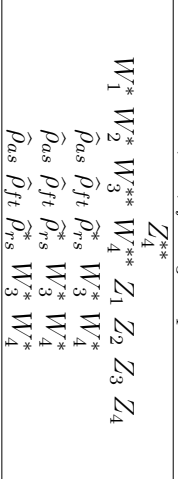 & 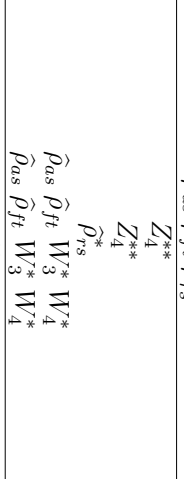 & 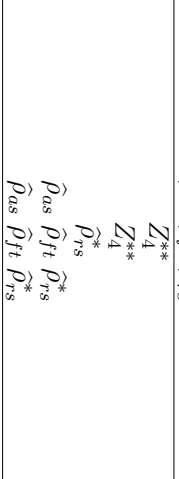 & 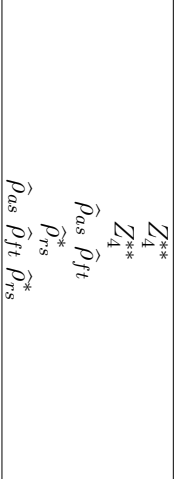 & 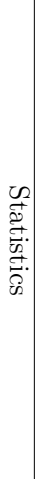 & \\
\hline 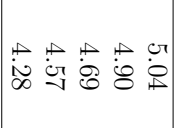 & 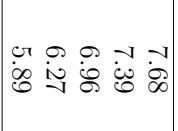 & 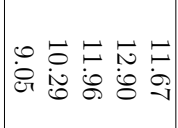 & 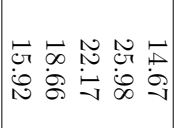 & 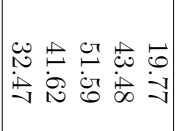 & 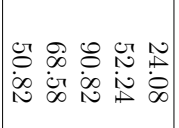 & 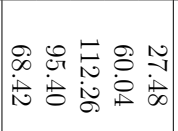 & 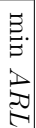 & \\
\hline 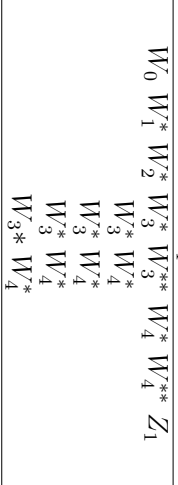 & (1) & & & & & & 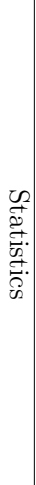 & $\frac{1}{4}$ \\
\hline 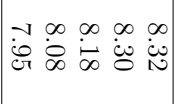 & 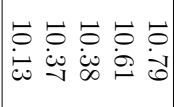 & 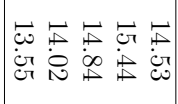 & 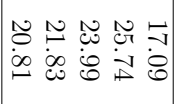 & 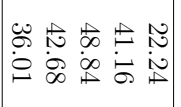 & 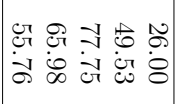 & 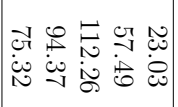 & $E$ & \\
\hline
\end{tabular}

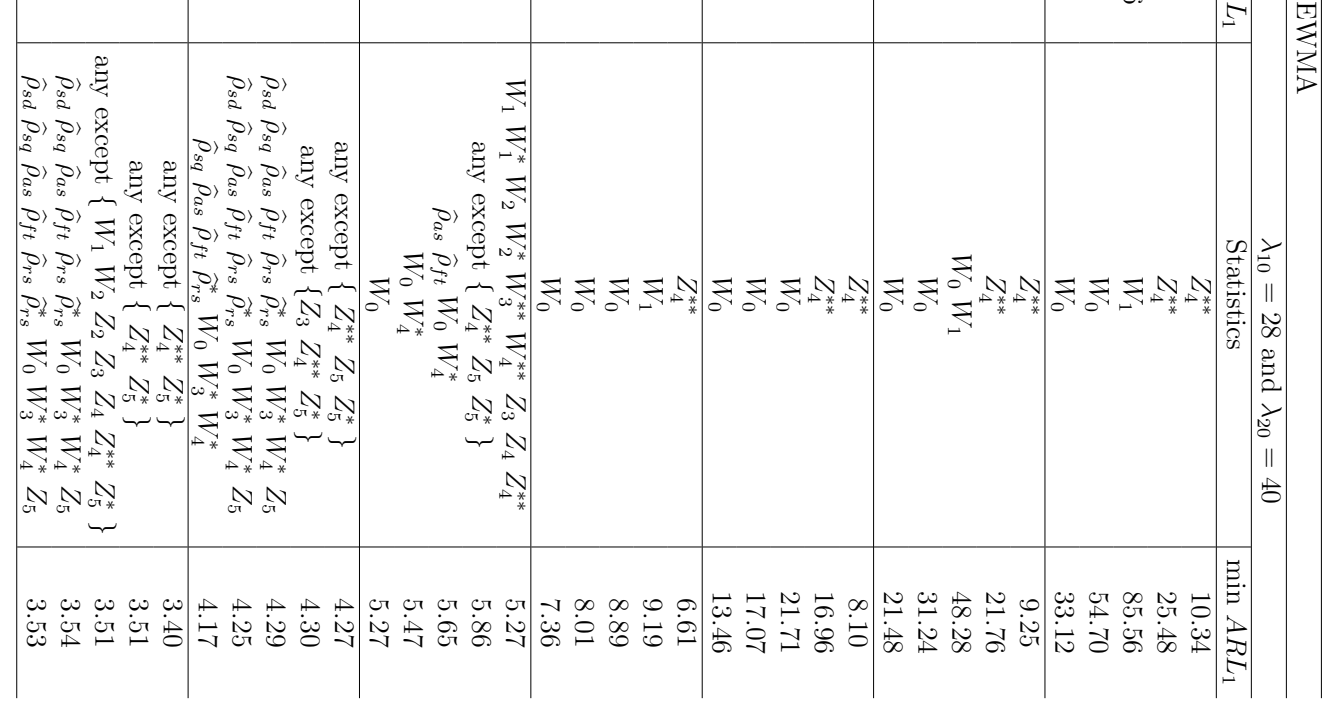




\section{Numerical Example}

In this chapter, a numerical example is presented to illustrate a real application.

Printing machine sales companies have rental contracts of printing machine for the customers. The rental contract usually consists of a fixed monthly fee and a cost per page fee.

In this case, the maintenance service with spare parts and consumption of consumables are included in the contract fees. So, the printing machine sales company should send spare parts and consumables to the customers by their costs. The control of spare parts and consumables is crucial as it impacts on the profitability of each contract. For example, the profitability will be worse if the company continues sending consumables items more than necessary.

In this numerical example, we focus on the delivery control of toner. There are 4 types of toner: Black (K toner), Yellow (Y toner), Magenta (M toner) and Cyan (C toner). The theoretical proportions of consumption of 4 types of toner as the print coverage of each color are defined in contract. Usually Y, M and C toners are more expensive than $\mathrm{K}$ toner. If the proportions of $\mathrm{Y}, \mathrm{M}$ and $\mathrm{C}$ toners' consumption against $\mathrm{K}$ toner's one increase, the profitability will be worse. So the ratio of $\mathrm{K}$ toner delivery and the other toners delivery is worth to be monitored in order to control it.

In this numerical example, we use a specific rental contract customer case of printing machine company and monitor the ratio of $\mathrm{C}$ and $\mathrm{K}$ delivery quantities $(\mathrm{C}$ per $\mathrm{K}$ ) by EWMA-type control chart with the statistic $Z 4^{* *}$. The customer contracted six rental machines for printing activity in November 2017 and the printing machine sales company has provided toners until June 2019. The C and K toners' standard monthly delivery quantities can be estimated by the defined coverage in contract and print volume which was researched and supposed for quotation. These standard quantities can be used as averages of delivery of each toner. Let $\lambda_{10}$ and $\lambda_{20}$ be in-control Conditions shown in Table 12. Then the index $\rho_{0}$ which is ratio of $\lambda_{10}$ and $\lambda_{20}$ is obtained. Our interest is to detect the increase of the $\rho_{0}$. EWMA constant is selected as 0.4 and $U C L$ is set with 3,04993 by Monte Carlo simulation considering the in-control rates.

Table 12 shows the historical delivery of $\mathrm{C}$ and $\mathrm{K}$ toners, the statistic $Z 4^{* *}$ of each sample and the statistic EWMA built with $Z 4^{* *}$. Figure 4 demonstrates EWMA-type control chart with UCL (red line). As a result, EWMA on 14th month surpassed $U C L$ and assignable case is recognized.

However, as the control chart was not implemented at the printing machine sales 
Table 12 - Example conditions and results

\section{Conditions}

\begin{tabular}{cr}
\hline$\lambda_{10}(\mathrm{C}$ Toner $)$ & 6.406 \\
\hline$\lambda_{20}(\mathrm{~K}$ Toner $)$ & 17.733 \\
\hline$\rho_{0}($ Index $)$ & 0.361 \\
\hline EWMA Constant & 0.400 \\
\hline UCL & $\mathbf{3 . 0 4 9 9 3}$ \\
\hline
\end{tabular}

Results

\begin{tabular}{|r|r|r|r|r|}
\hline Month & C toner & K toner & Z4** & EWMA with Z4** \\
\hline 1 & 13 & 13 & 2.87 & 1.150 \\
\hline 2 & 3 & 10 & -0.36 & 0.547 \\
\hline 3 & 11 & 10 & 2.76 & 1.434 \\
\hline 4 & 5 & 0 & 2.74 & 1.957 \\
\hline 5 & 8 & 25 & -0.40 & 1.014 \\
\hline 6 & 5 & 0 & 2.74 & 1.705 \\
\hline 7 & 3 & 3 & 1.23 & 1.514 \\
\hline 8 & 8 & 12 & 1.53 & 1.520 \\
\hline 9 & 5 & 16 & -0.37 & 0.764 \\
\hline 10 & 5 & 2 & 2.30 & 1.377 \\
\hline 11 & 24 & 25 & 3.92 & 2.396 \\
\hline 12 & 21 & 22 & 3.64 & 2.892 \\
\hline 13 & 16 & 25 & 2.14 & 2.590 \\
\hline 14 & 47 & 60 & 4.81 & $\mathbf{3 . 4 7 6}$ \\
\hline 15 & 21 & 32 & 2.57 & $\mathbf{3 . 1 1 2}$ \\
\hline 16 & 23 & 35 & 2.70 & 2.948 \\
\hline 17 & 19 & 32 & 2.10 & 2.609 \\
\hline 18 & 16 & 25 & 2.14 & 2.420 \\
\hline
\end{tabular}

company, it only perceived abnormality lately at 18th month when the company required to renew the contract. The company searched and recognized that the toner delivery amounts were extremely high since 11th month however in customer's stock there are no excess toners (which means they were disappeared/missed or even stolen). The company could consider a shift in the ratio of $\mathrm{C}$ and $\mathrm{K}$ quantities from 11th month because the toners might be disappeared with different proportion. The control chart detected the change in 14th month which leads a run length of 4 months. Although this is not an ideal run length, but, it is much better than the discovery of abnormality only in 18th month as the company could notice the problem four months earlier as also could minimize the loss related to the toners if it had implemented a control chart. For this extraordinary problem, one may question why not a control chart to monitor the individual toner delivery quantity (for example, only $\mathrm{C}$ toner delivery quantity) as the quantities increased significantly. The main reason is that it may not be really informative if this increase may come from a print volume hike (which results a better profit as it increases the revenue by cost per click fee). In this example, the control chart for the ratio of two toner delivery quantities could give a signal related to the change of proportion of consumption earlier than the company's recognition. 


\section{EWMA-type Control Chart}

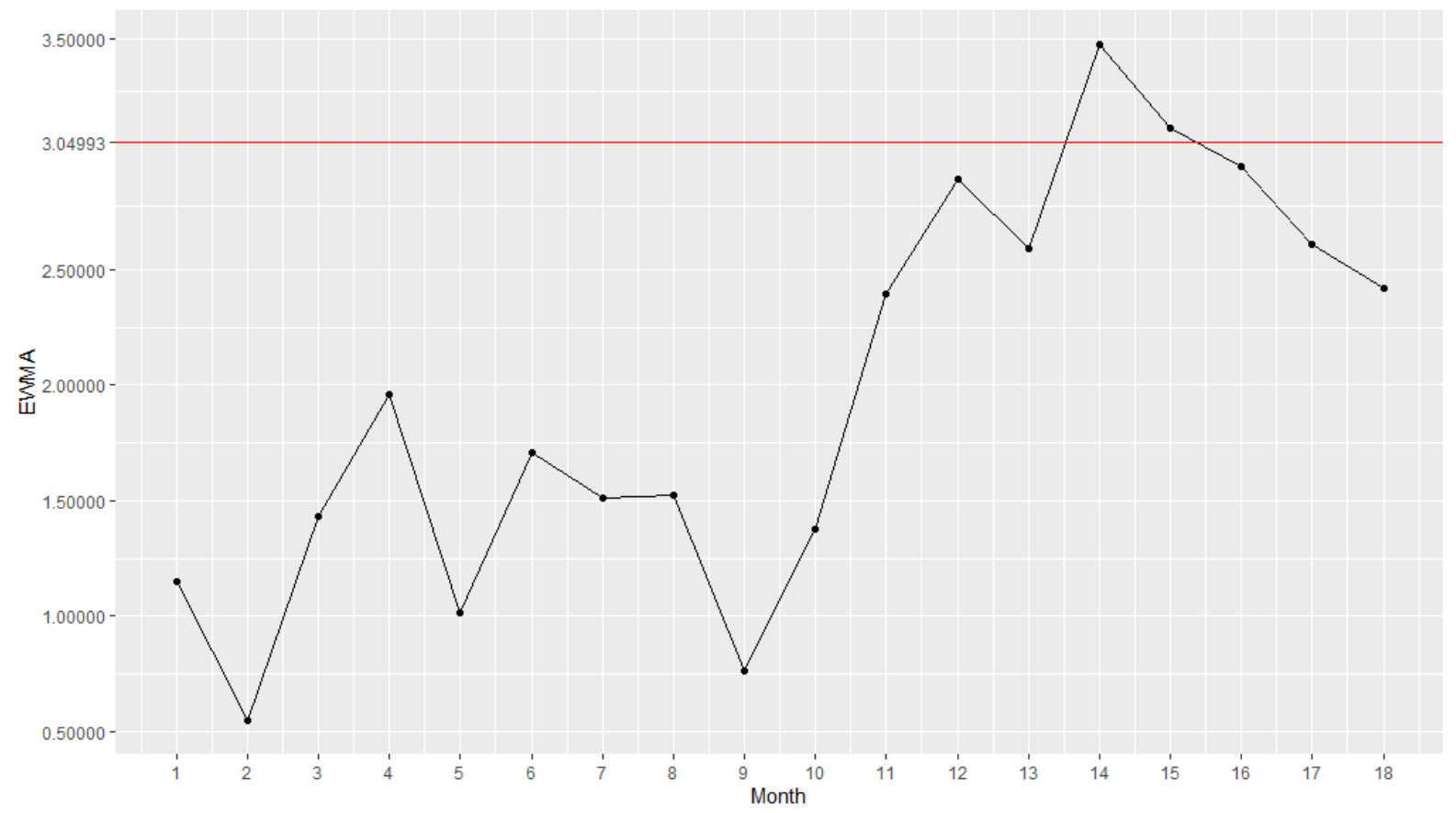

Figure 4 - EWMA-type Control Chart with $Z 4^{* *}$ for numerical example 



\section{Final Remarks}

In this study the performances of the control charts Shewhart and EWMA for monitoring the ratio of two Poisson rates are evaluated by an extensive simulation study. Several combination of statistics and normal transformations are evaluated.

EWMA control chart really improves the performance as expected. So the recommendation is for its use. The choice of the statistics depends on the three inputs: the in-control Poisson rates, the shifts on the ratio and in-control ratio. In summary, a practical recommendation: when the difference between the two in-control Poisson rates is large use the statistic $Z_{4}^{* *}$ for small shifts on the ratio and small increases on the average rate otherwise use the statistic $W_{0}$. If the diffrence between the two in-control Poisson rates is small, the statistic $W_{4}^{*}$ could be used instead of $W_{0}$.

In case Shewhart control chart should be used, the statistics as $\hat{\rho}_{a s}$ and $\hat{\rho}_{f t}$ can be chosen for decreases on the average Poisson rate. For other cases, the best is to run a simulation study to choose the best set of statistics.

Certainly the values of $A R L_{1}$ are still high (lowest values are around 3) and more research is needed to improve the performance. Result is not ideal as only individual observations are available to build control chart. For future study, one possibility is to consider the development of CUSUM-type chart. 



\section{Bibliography}

ANSCOMBE, F. The transformation of poisson, binomial and negative-binomial data. Biometrika, v. 35, p. 246-254, 1948. Cited on page 24.

BARTLETT, M. The square root transformation in analysis of variance. Supplement to the Journal of the Royal Statistical Society, v. 3, p. 68-78, 1936. Cited on page 24.

BROOK, D.; EVANS, D. An approach to the probability distribution of cusum run length. Biometrika, Oxford University Press, v. 59, n. 3, p. 539-549, 1972. Cited on page 20 .

DODGE H. F.; ROMIG, H. G. A method of sampling inspection. Bell System Technical Journal, v. 8.4, p. 613-631, 1929. Cited on page 17.

FREEMAN MURRAY F.; TUKEY, J. W. Transformations related to the angular and the square root. The Annals of Mathematical Statistics, v. 21, p. 607-611, 1950. Cited on page 24 .

GU K.; NG, H. T. M. S. W. Testing the ratio of two poisson rates. Biometrical Journal, v. 50(2), p. 283-298, 2008. Cited 2 times on pages 21 and 22.

HUFFMAN, M. An improved approximate two-sample poisson test. Applied Statistics, v. 33, p. 224-226, 1984. Cited on page 24.

MONTGOMERY, D. C. Introduction to Statistical Quality Control. Hoboken, NJ, USA: John Wiley and Sons, inc., 2005. Cited on page 17.

MOOD, A.; GRAYBILL, F.; DUANE, C. Introduction to the Theory of Statistics. [S.1.]: New York: McGraw Hill, 1974. Cited on page 26.

PAGE, E. S. Continuous inspection schemes. Biometrika, v. 41, p. 100-115, 1954. Cited on page 18 .

R Core Team. R: A Language and Environment for Statistical Computing. Vienna, Austria, 2017. Available from Internet: <http://www.R-project.org/>. Cited on page 27.

ROBERTS, S. W. Control chart tests based on geometric moving averages. technometrics, v. 1, p. 239-250, 1959. Cited on page 18.

ROSSI G.; LAMPUGNANI, L. . M. M. An approximate cusum procedure for surveillance of health events. Statistics in Medicine, v. 18, p. 2111-2122, 1950. Cited on page 25.

SHEWHART, W. C. Some applications of statistical methods to the analysis of physical and engineering data. Bell System Technical Journal, v. 3.1, p. 43-87, 1924 . Cited on page 17 . 

Appendix 



\section{APPENDIX A - Source code of stage I}

$<$ Data set of random variables $\mathrm{X}$ and $\mathrm{Y}>$

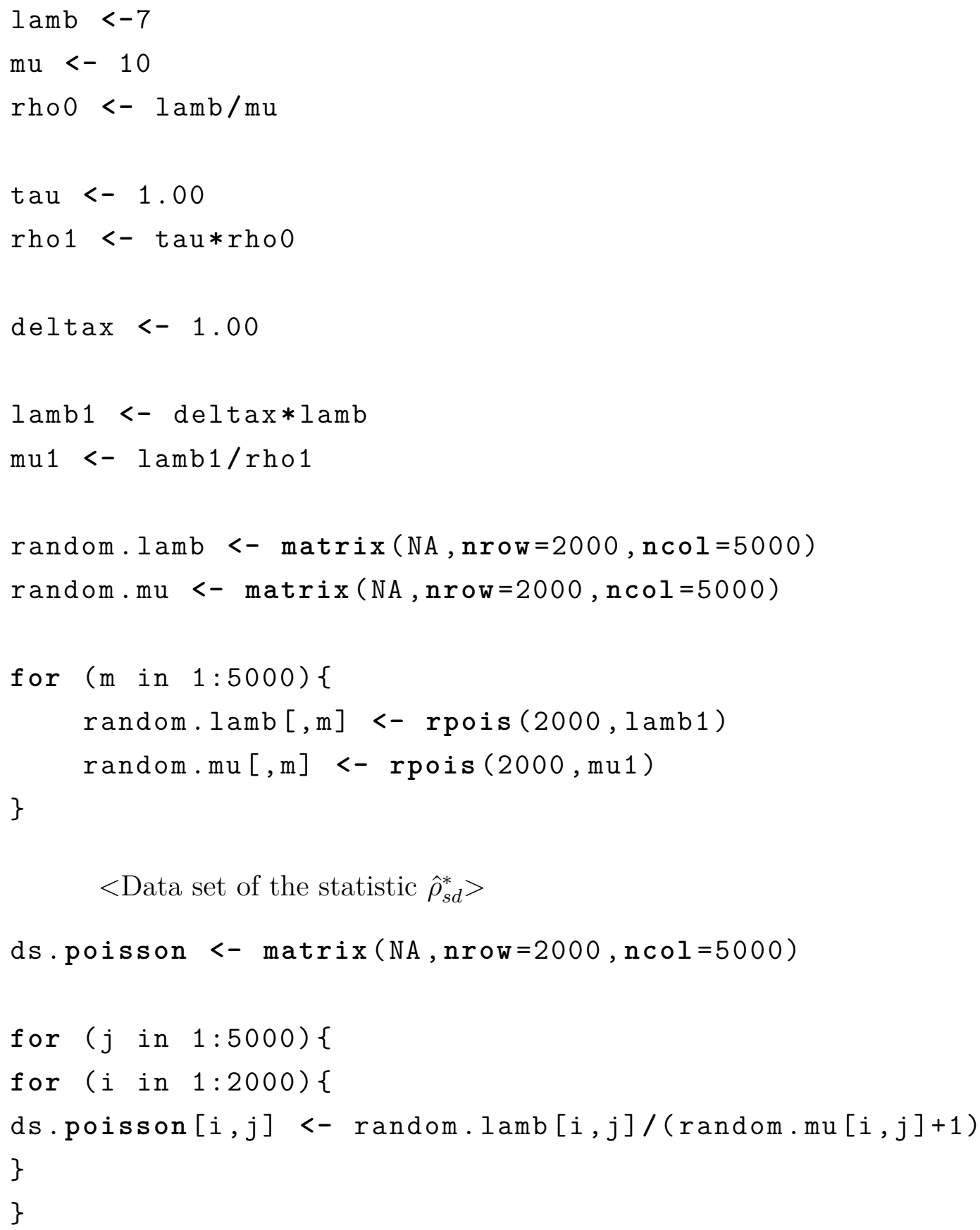

$<$ Data set of the statistic $\hat{\rho}_{s q}^{*}>$ 


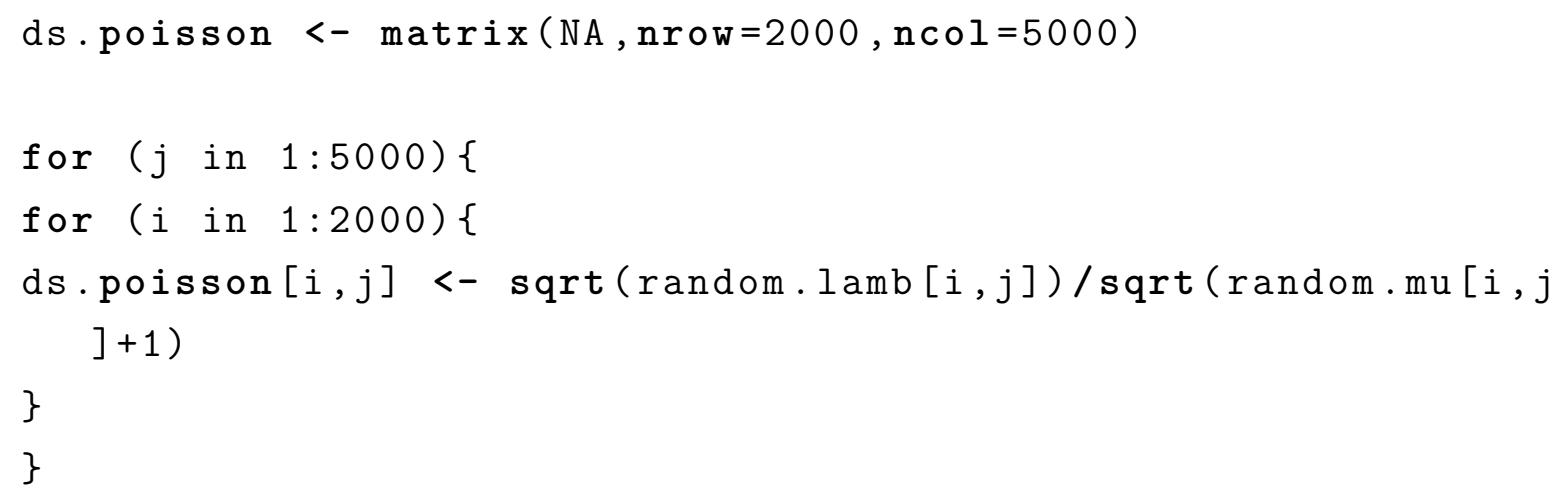

$<$ Data set of the statistic $\hat{\rho}_{a s}>$

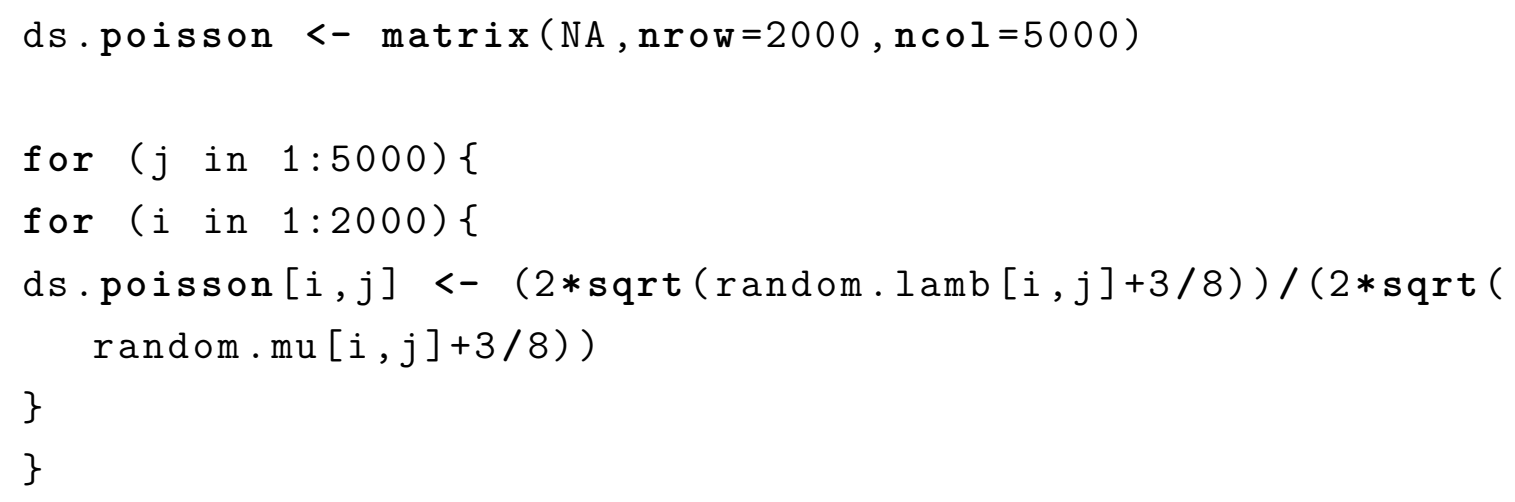

$<$ Data set of the statistic $\hat{\rho}_{f t}>$

ds.poisson <- matrix (NA, nrow $=2000$, ncol=5000)

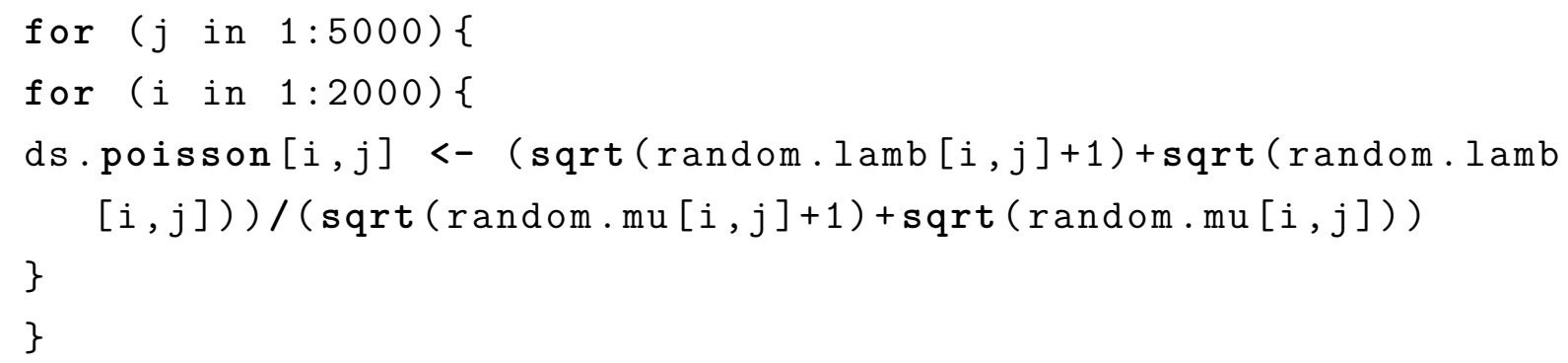

$<$ Data set of the statistic $\hat{\rho}_{r s}^{*}>$

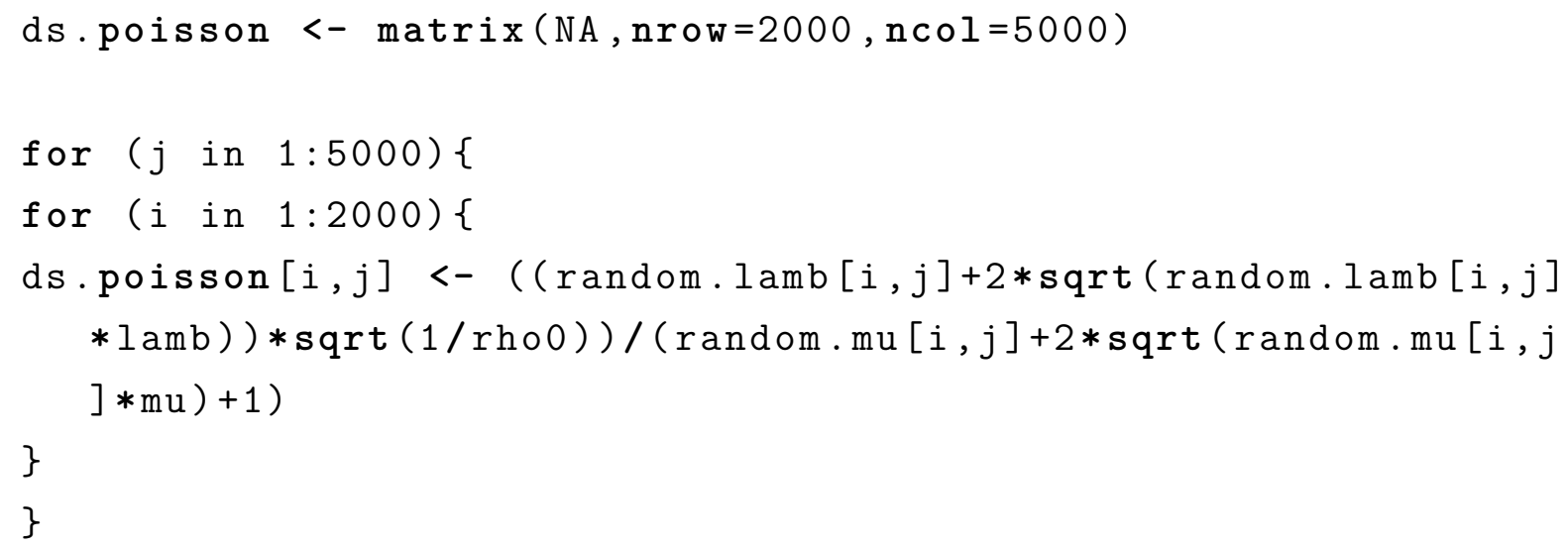


$<$ Data set of the statistic $\hat{\rho}_{r s}^{* *}>$

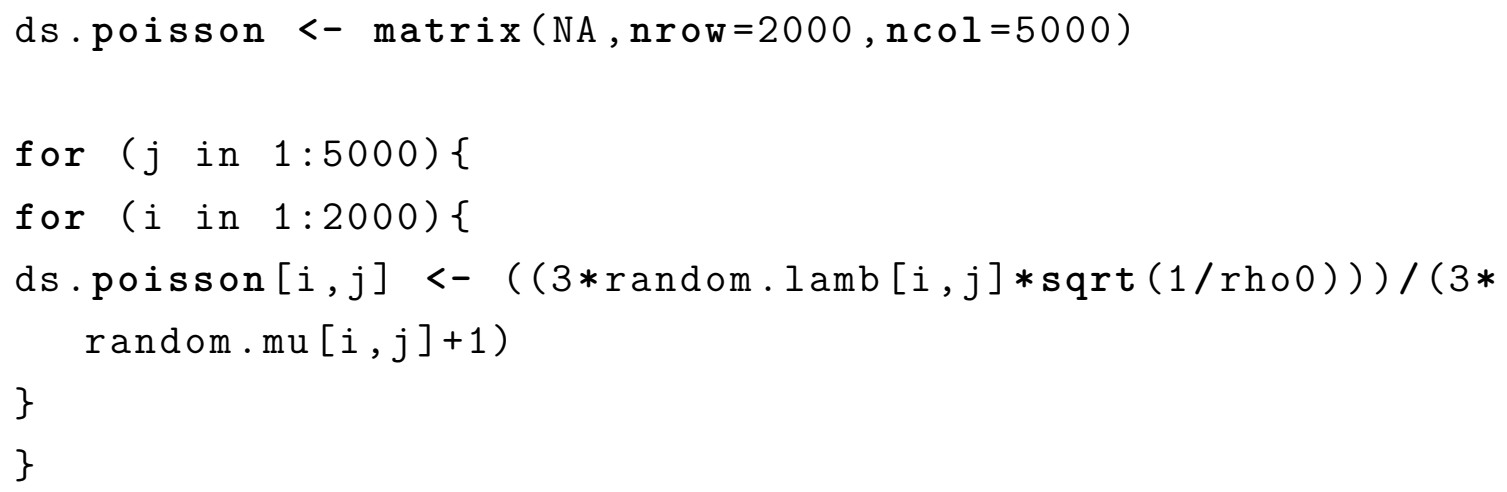

$<$ Data set of the statistic $W_{0}>$

ds.poisson <- matrix (NA, nrow $=2000$, ncol=5000)

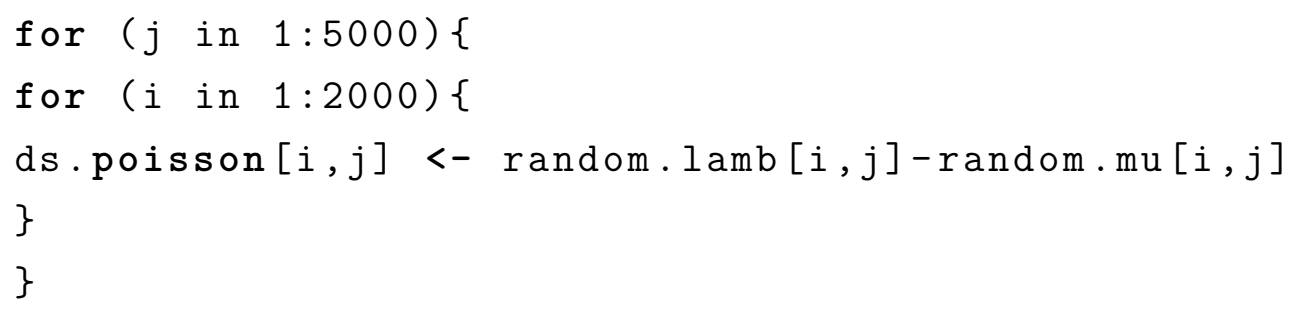

$<$ Data set of the statistic $W_{1}>$

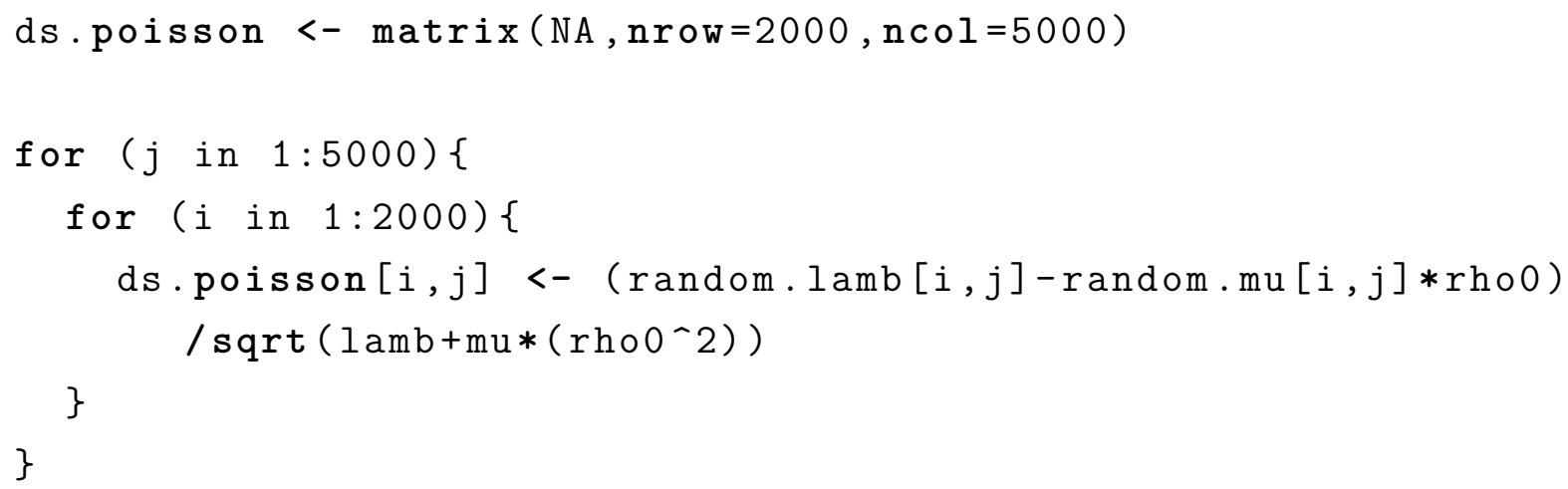

$<$ Data set of the statistic $W_{1}^{* *}>$

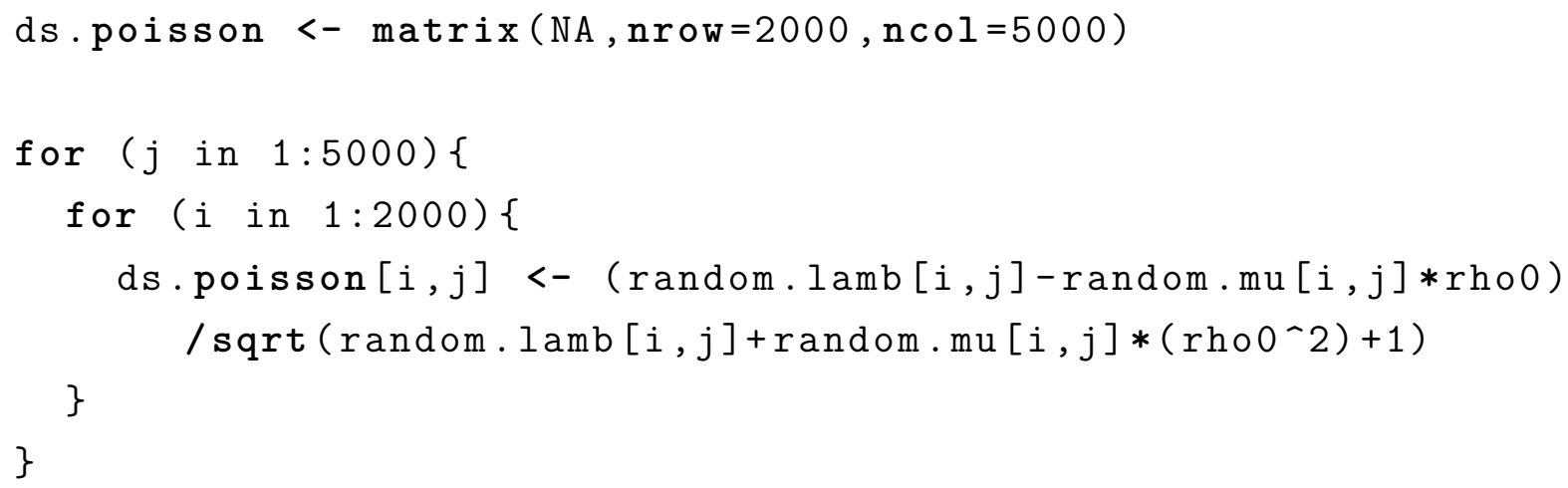

$<$ Data set of the statistic $W_{2}>$ 


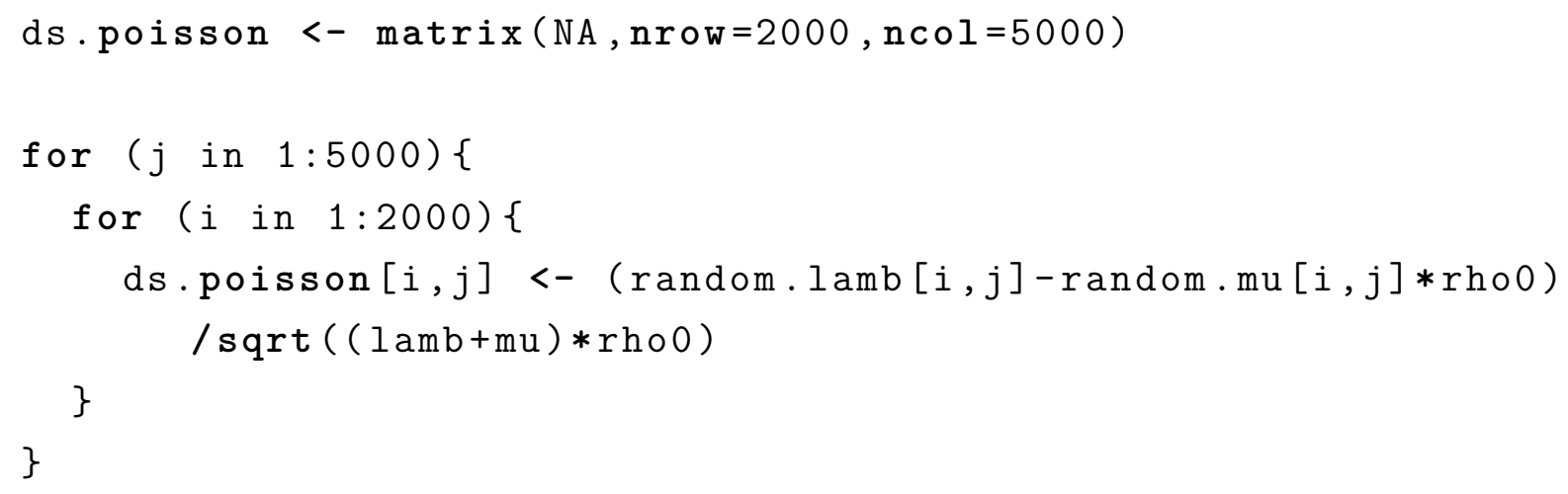

$<$ Data set of the statistic $W_{2}^{* *}>$

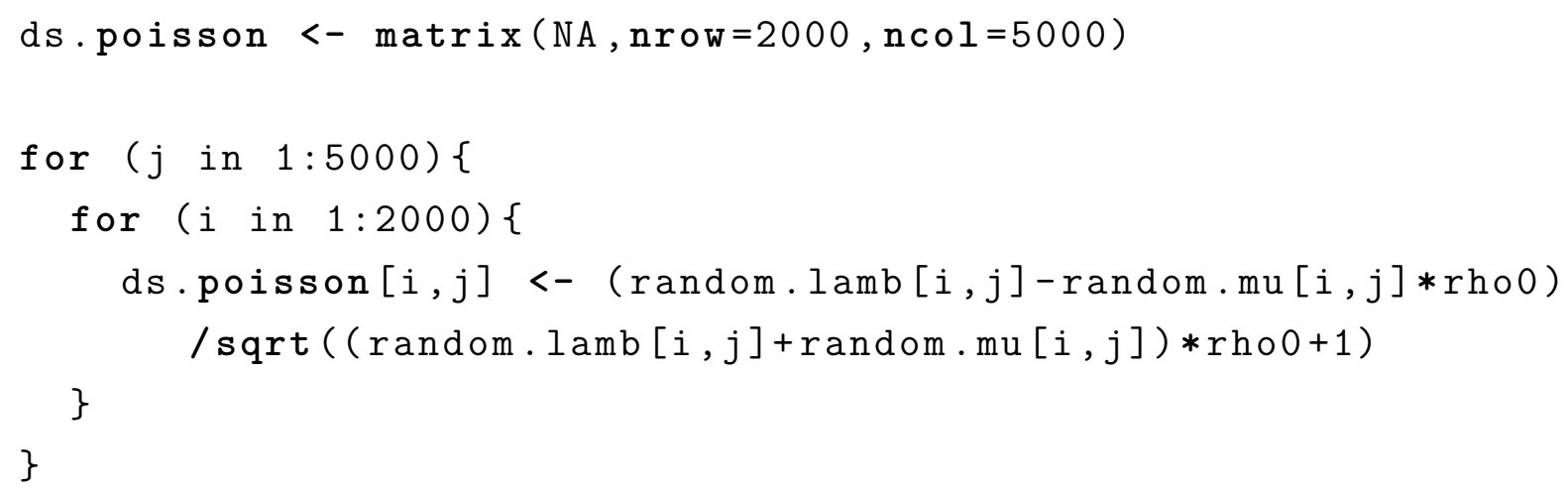

$<$ Data set of the statistic $W_{3}^{\star}>$

ds.poisson <- matrix (NA, nrow $=2000$, ncol=5000)

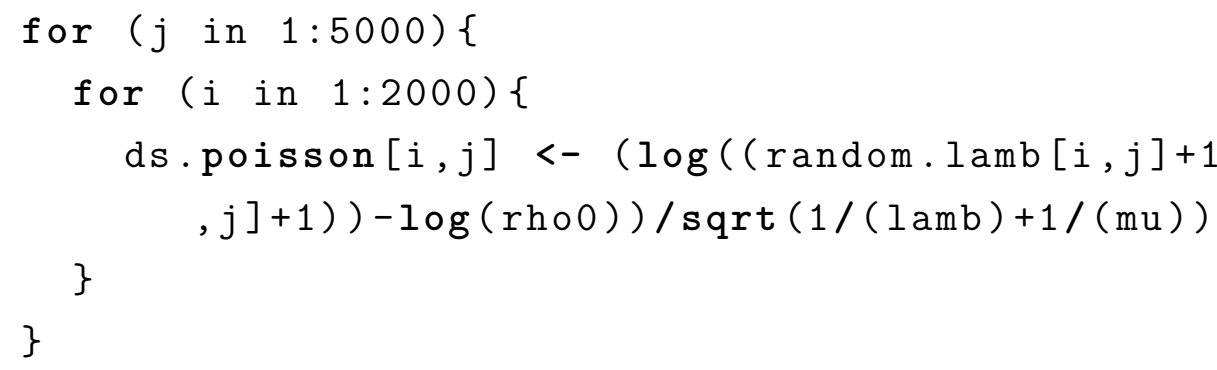

$<$ Data set of the statistic $W_{3}^{* *}>$

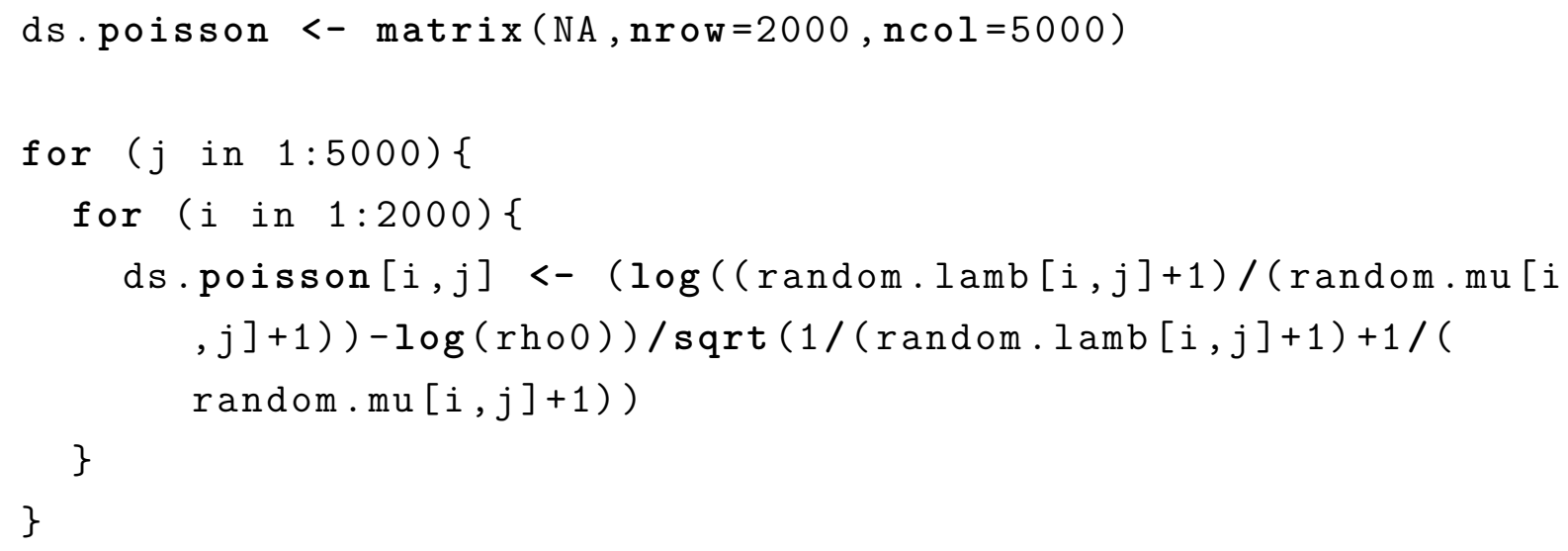


$<$ Data set of the statistic $W_{4}^{\star}>$

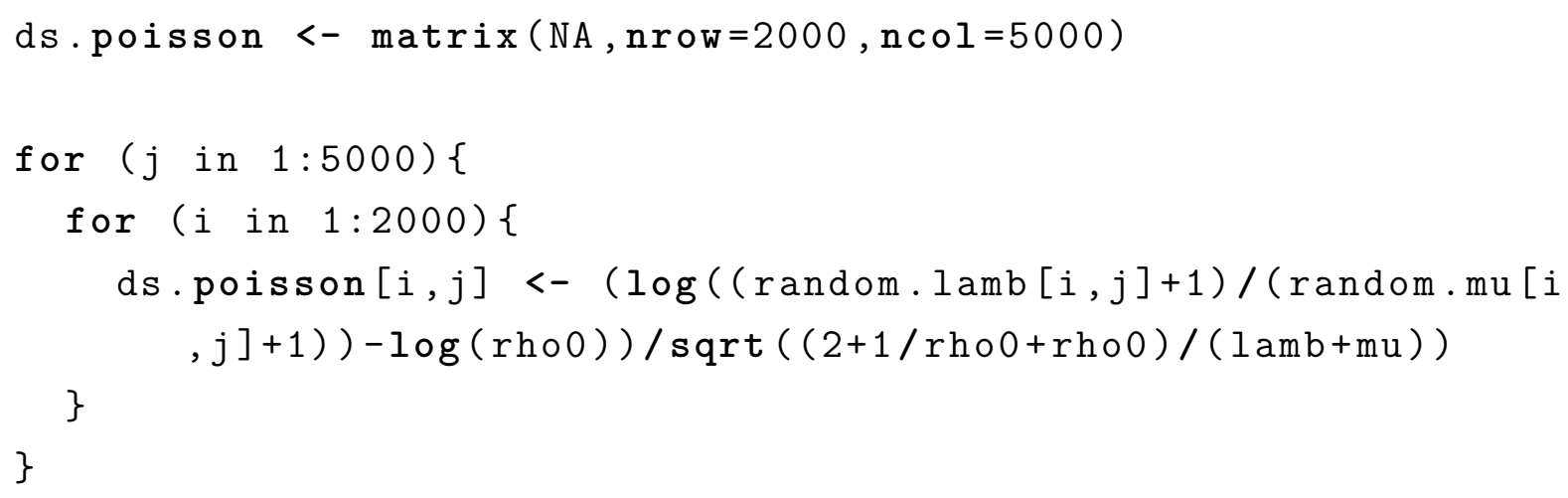

$<$ Data set of the statistic $W_{4}^{* *}>$

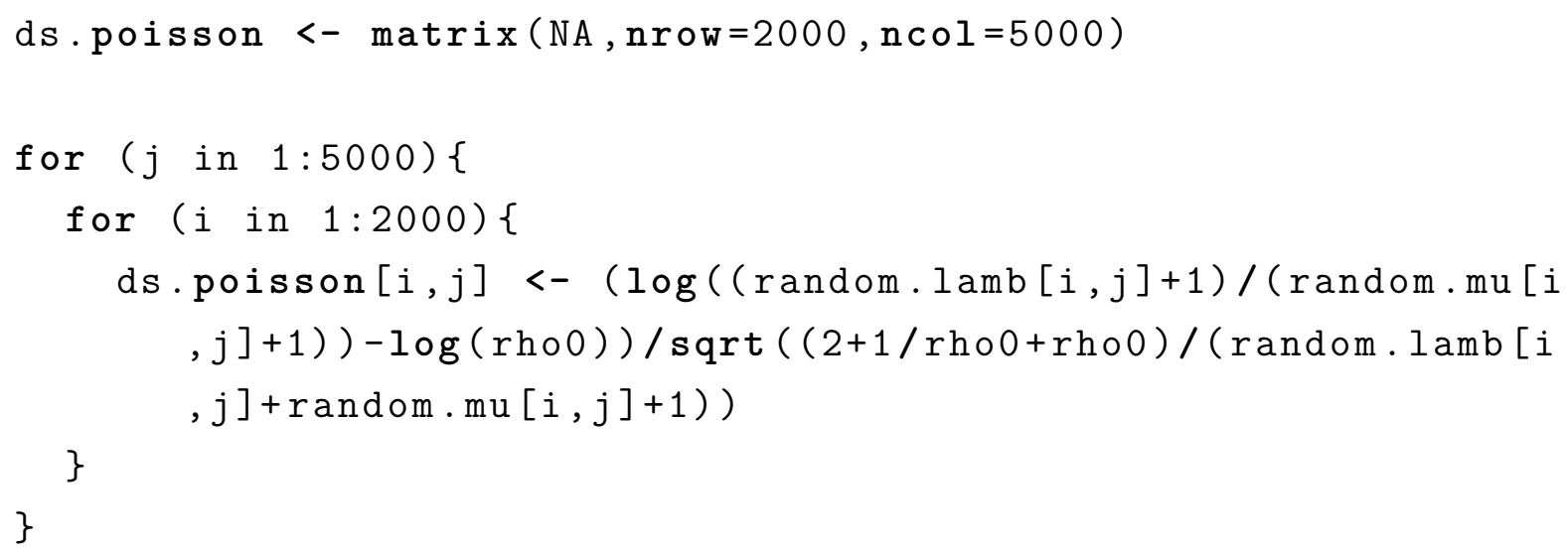

$<$ Data set of the statistic $Z_{1}>$

ds.poisson <- matrix (NA, nrow $=2000$, ncol=5000)

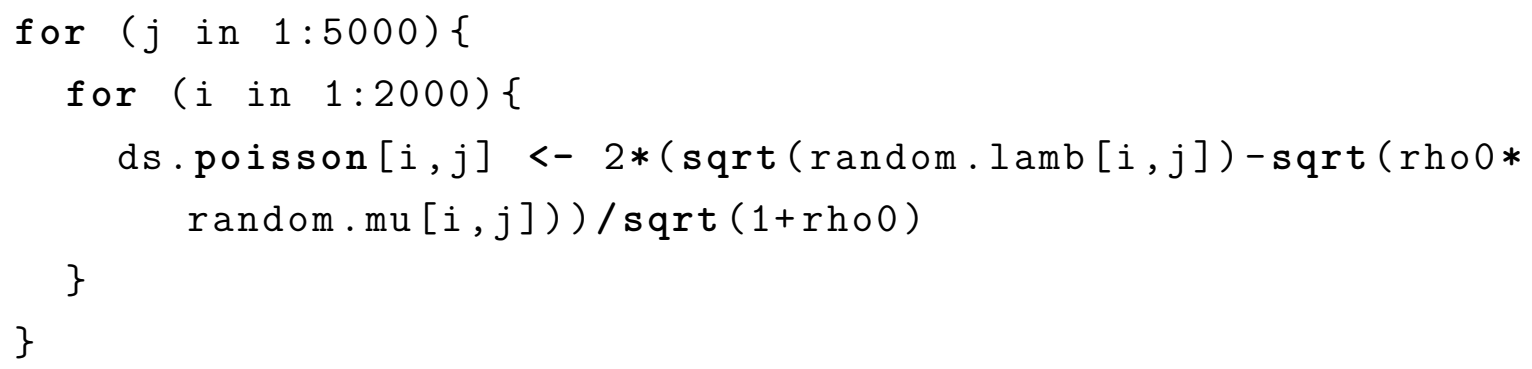

$<$ Data set of the statistic $Z_{2}>$

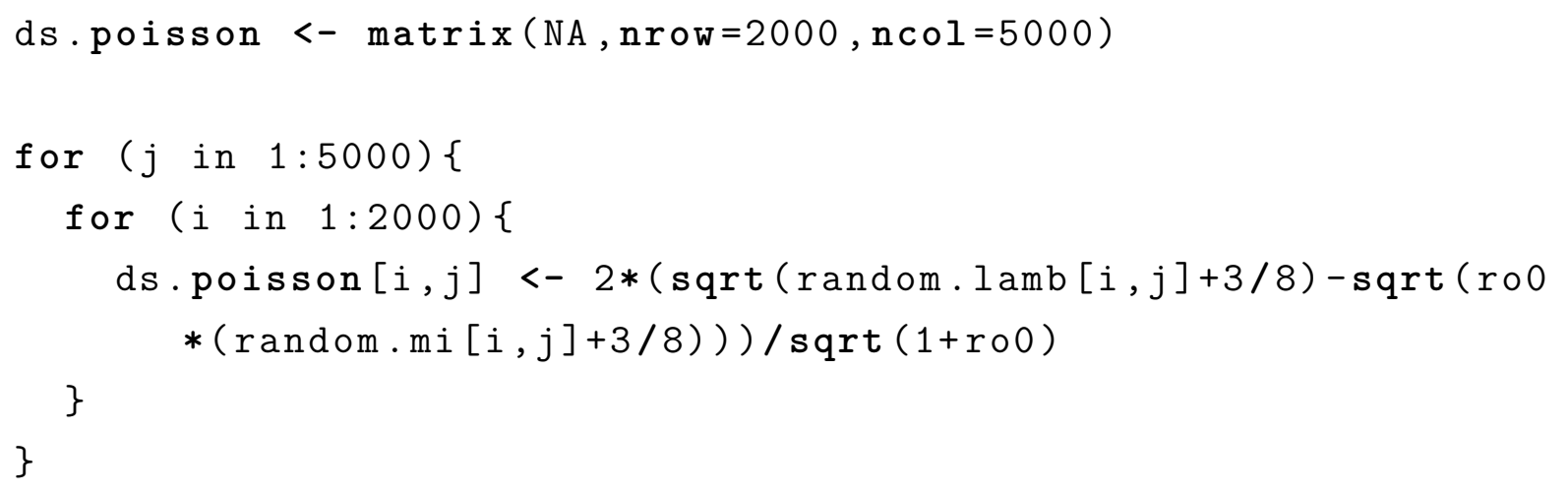


$<$ Data set of the statistic $Z_{3}>$

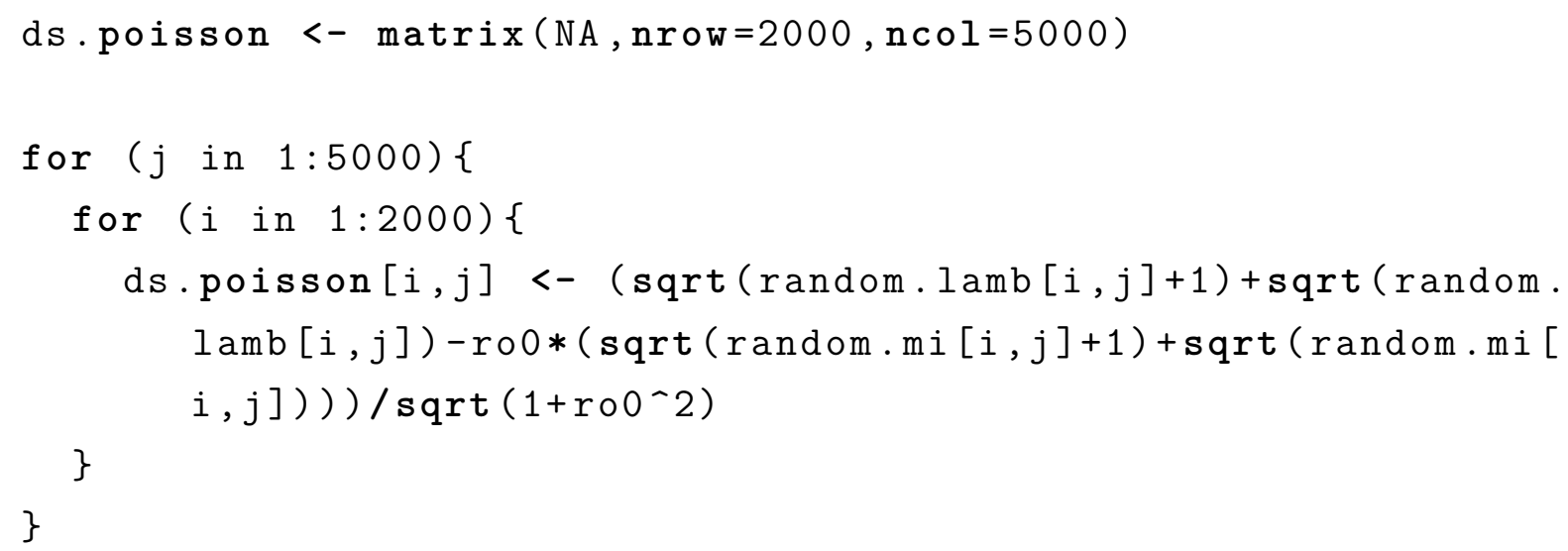

$<$ Data set of the statistic $Z_{4}>$

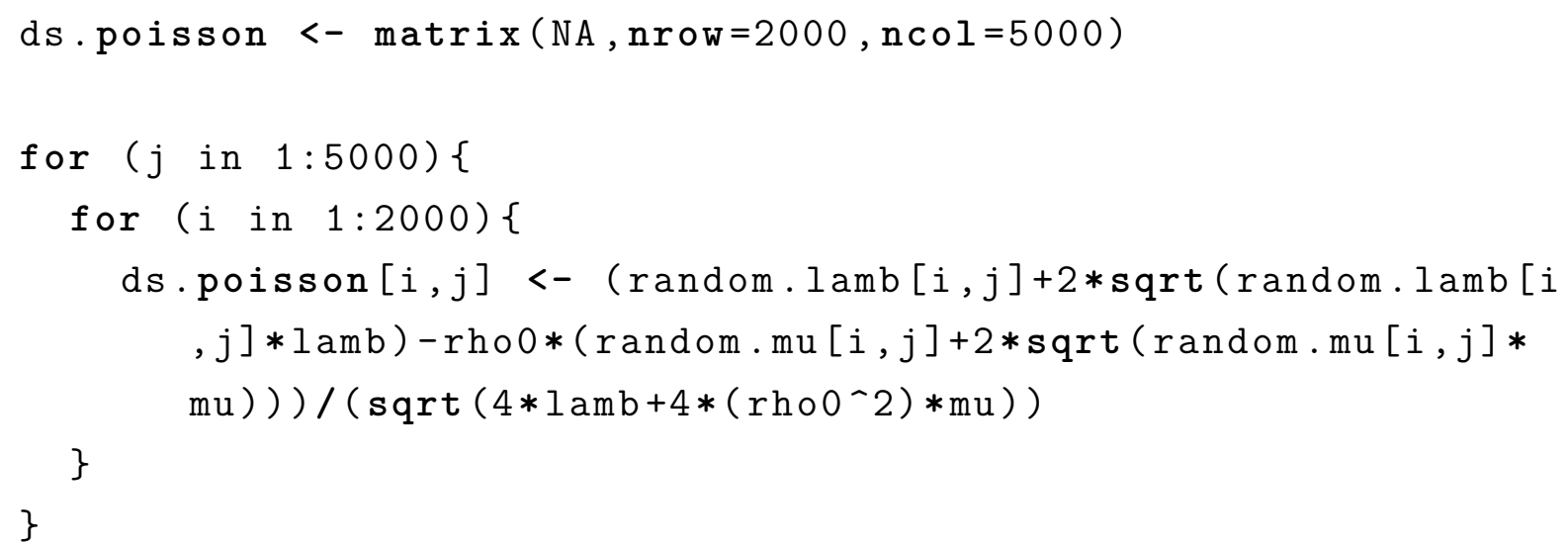

$<$ Data set of the statistic $Z_{4}^{* *}>$

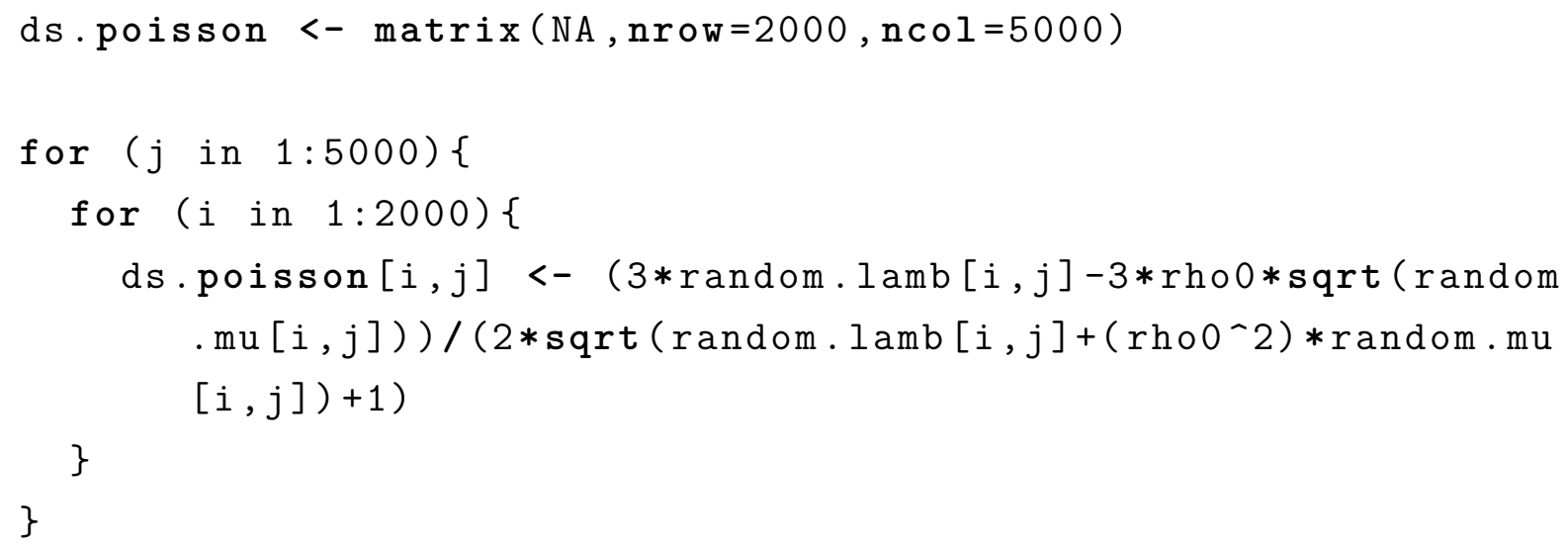

$<$ Data set of the statistic $Z_{5}>$

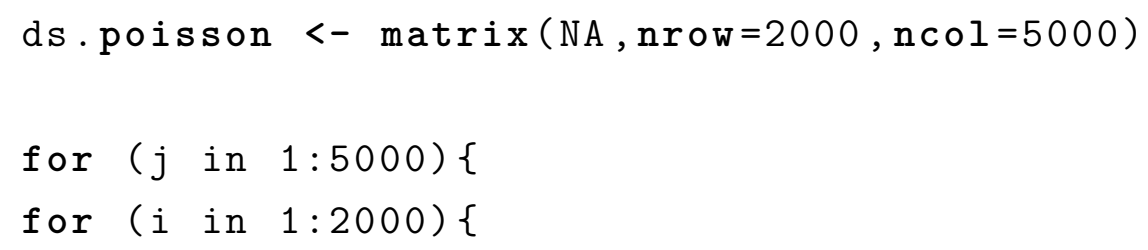




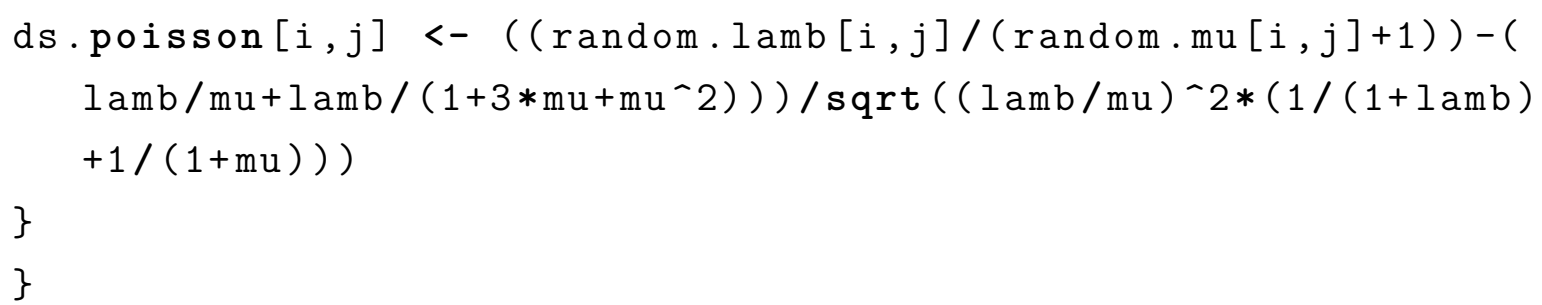

$<$ Data set of the statistic $Z_{5}^{*}>$

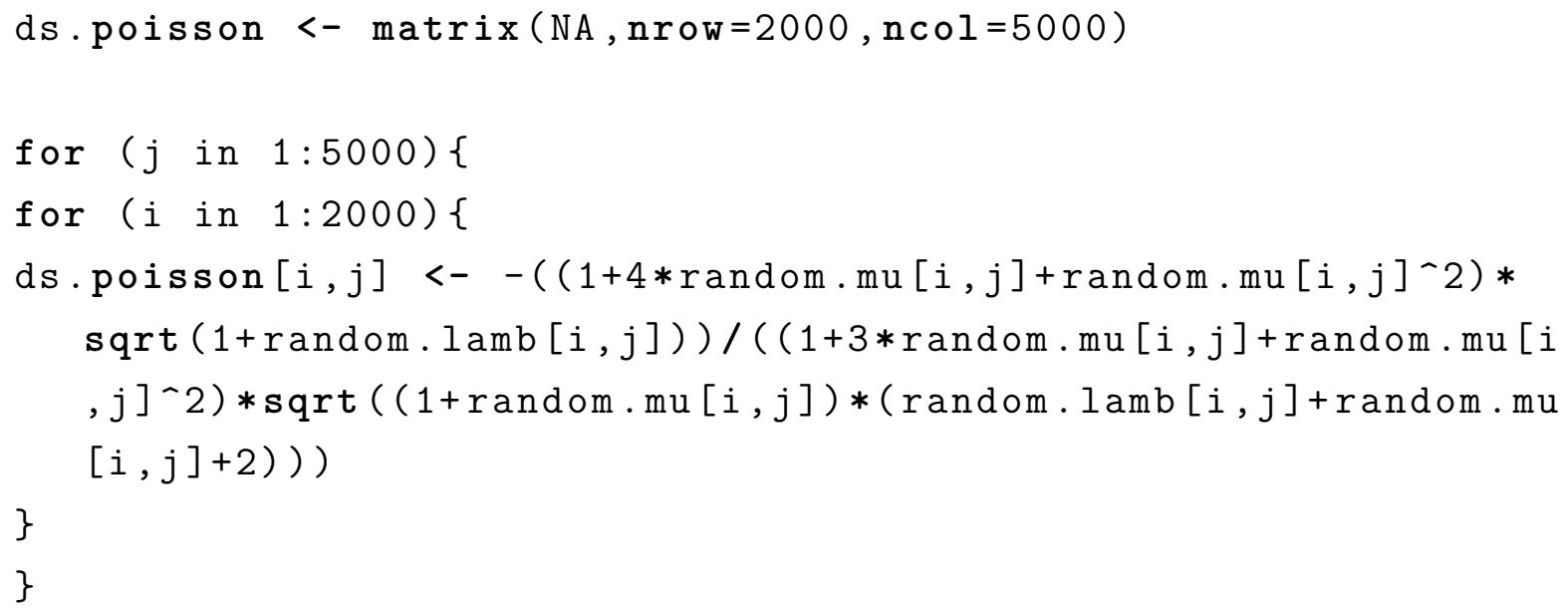

$<$ Data set of EWMA with the statistic $\hat{\rho}_{s d}^{*}>$

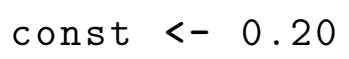

$<$ Data set of EWMA with the statistic $\hat{\rho}_{s q}^{*}>$

const $<-0.20$

ds. ewma.zi $<-\operatorname{matrix}(N A$, nrow $=2000$, ncol $=5000)$ 


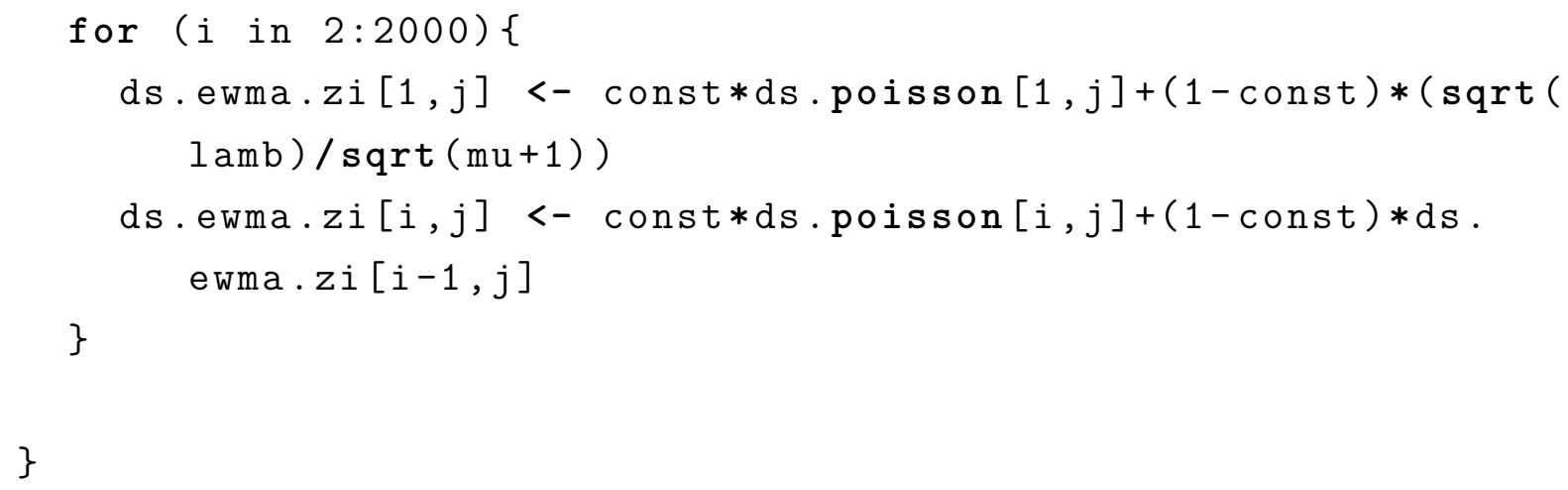

$<$ Data set of EWMA with the statistic $\hat{\rho}_{a s}>$

const $<-0.20$

ds. ewma.zi $<-\operatorname{matrix}(N A$, nrow $=2000$, ncol=5000)

for $(j$ in $1: 5000)\{$

for $(i$ in $2: 2000)\{$

ds.ewma.zi $[1, j]<-$ const $* d s$.poisson $[1, j]+(1-$ const $) *((2 *$ $\operatorname{sqrt}(1 \mathrm{amb}+3 / 8)) /(2 * \operatorname{sqrt}(\mathrm{mu}+3 / 8)))$

ds.ewma.zi $[i, j]<-$ const*ds.poisson $[i, j]+(1-$ const $) * d s$. ewma.zi $[i-1, j]$

\}

\}

$<$ Data set of EWMA with the statistic $\hat{\rho}_{f t}>$

const $<-0.20$

ds. ewma.zi $<-\operatorname{matrix}(N A$, nrow $=2000$, ncol $=5000)$

for $(j$ in $1: 5000)\{$

for ( $i$ in $2: 2000)\{$

ds.ewma.zi $[1, j]<-$ const*ds.poisson $[1, j]+(1-$ const $) *$ ( (sqrt

$(1 a m b+1)+\operatorname{sqrt}(1 a m b)) /(\operatorname{sqrt}(m u+1)+\operatorname{sqrt}(m u)))$

ds.ewma.zi[i,j] <- const*ds.poisson $[i, j]+(1-$ const $) * d s$.

ewma.zi $[i-1, j]$

\} 
\}

$<$ Data set of EWMA with the statistic $\hat{\rho}_{r s}^{*}>$

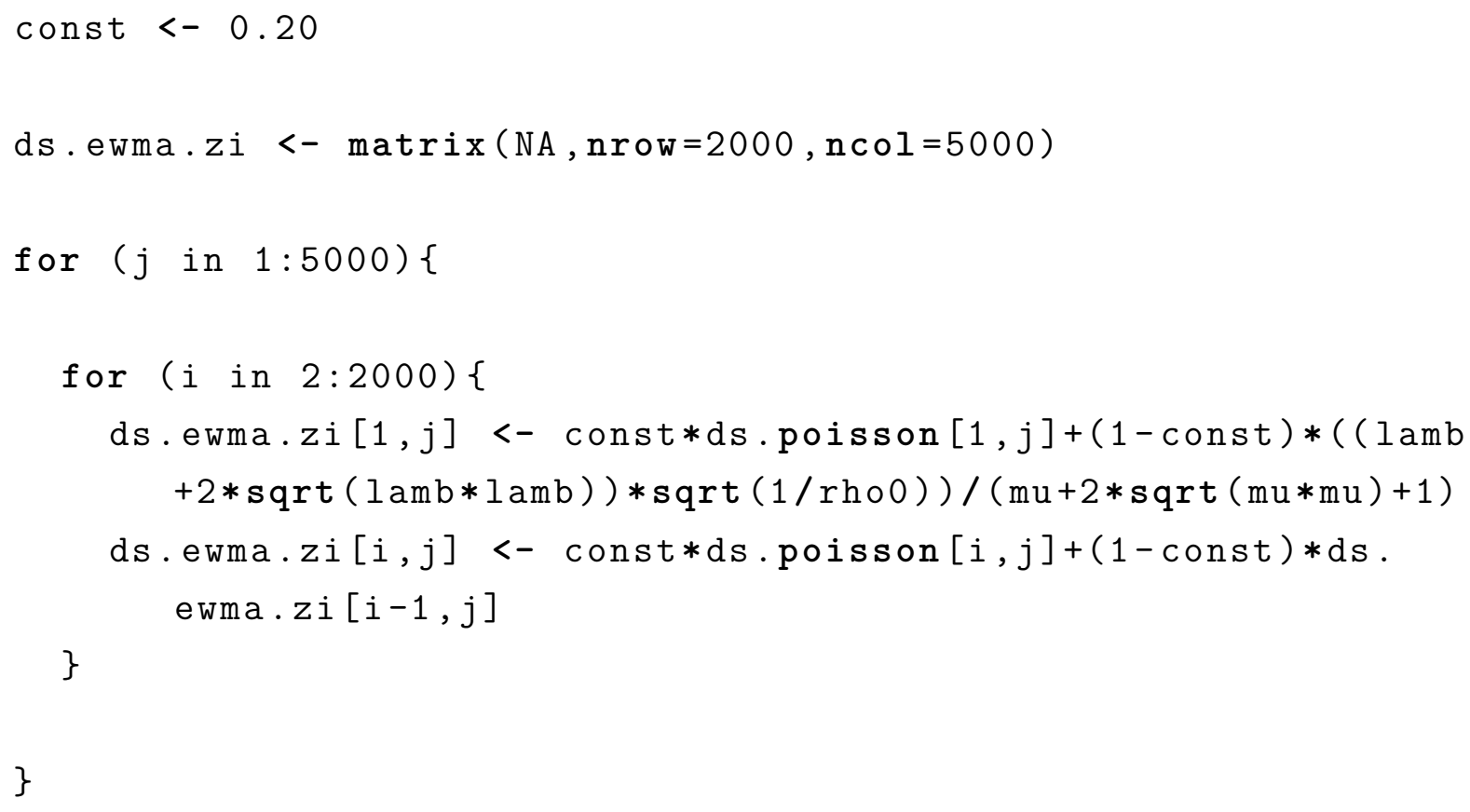

$<$ Data set of EWMA with the statistic $\hat{\rho}_{r s}^{* *}>$

const $<-0.20$

ds.ewma.zi $<-\operatorname{matrix}(N A$, nrow $=2000$, ncol=5000)

for $(j$ in $1: 5000)\{$

for ( $i$ in $2: 2000$ )

ds.ewma.zi $[1, j]<-$ const $* d s \cdot$ poisson $[1, j]+(1-$ const $) *(3 *$ lamb*sqrt $(1 / \mathrm{rho} 0)) /(3 * m u+1)$

\}

$<$ Data set of EWMA with the statistic $W_{0}>$

const $<-0.2$

ds.ewma.zi $<-\operatorname{matrix}(N A$, nrow $=2000$, ncol=5000)

for $(j$ in $1: 5000)\{$ 


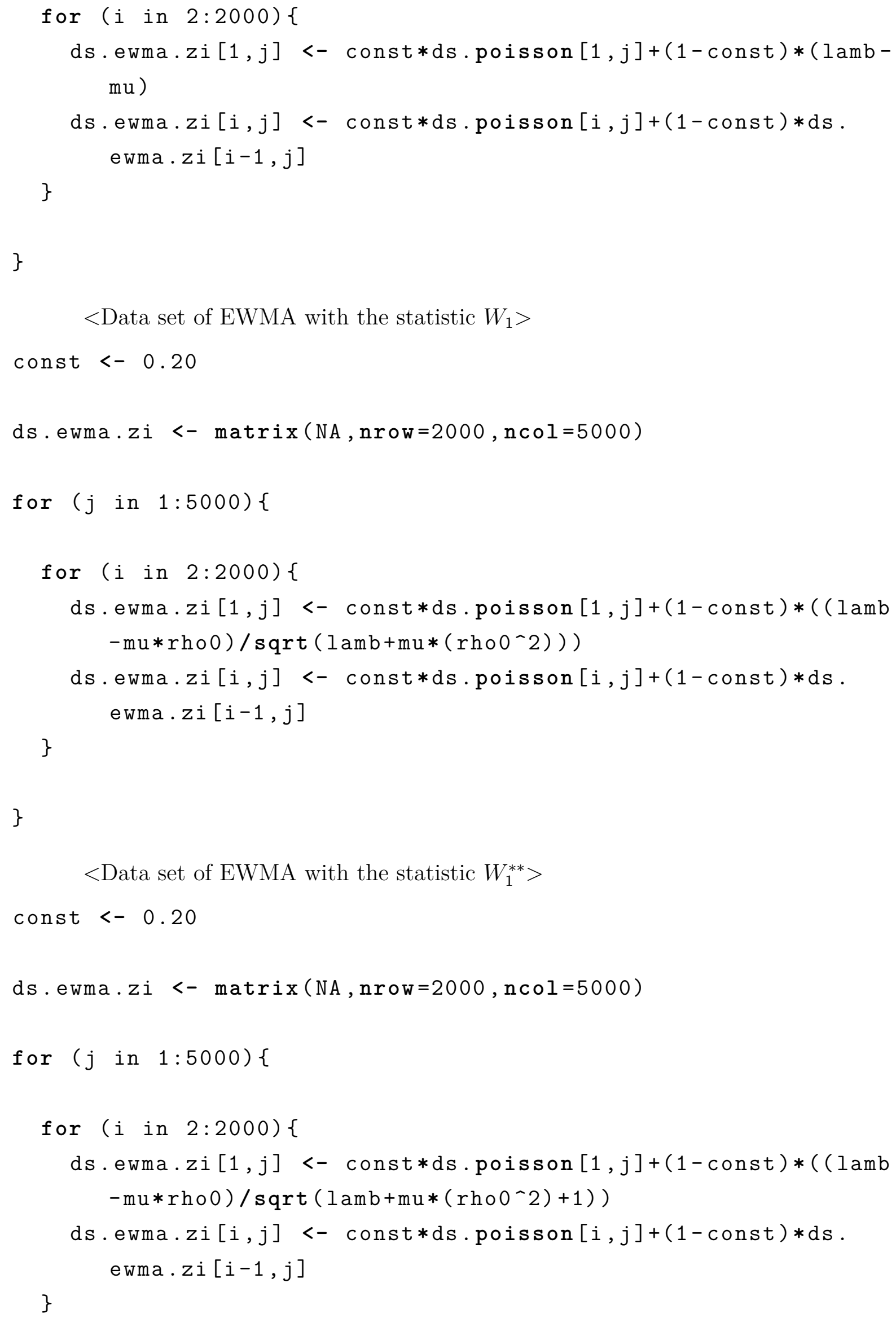


\}

$<$ Data set of EWMA with the statistic $W_{2}>$

$<$ Data set of EWMA with the statistic $W_{2}^{* *}>$

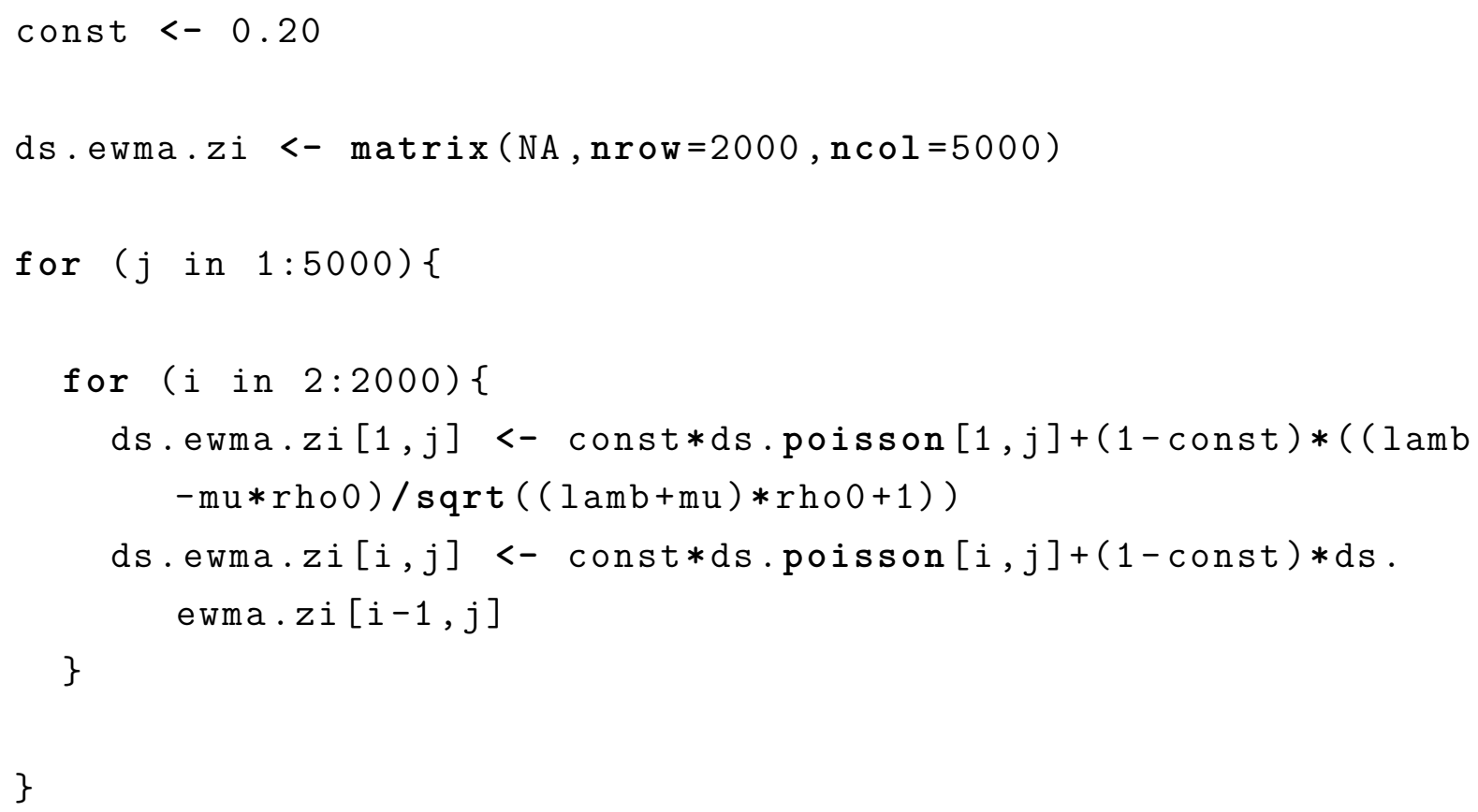




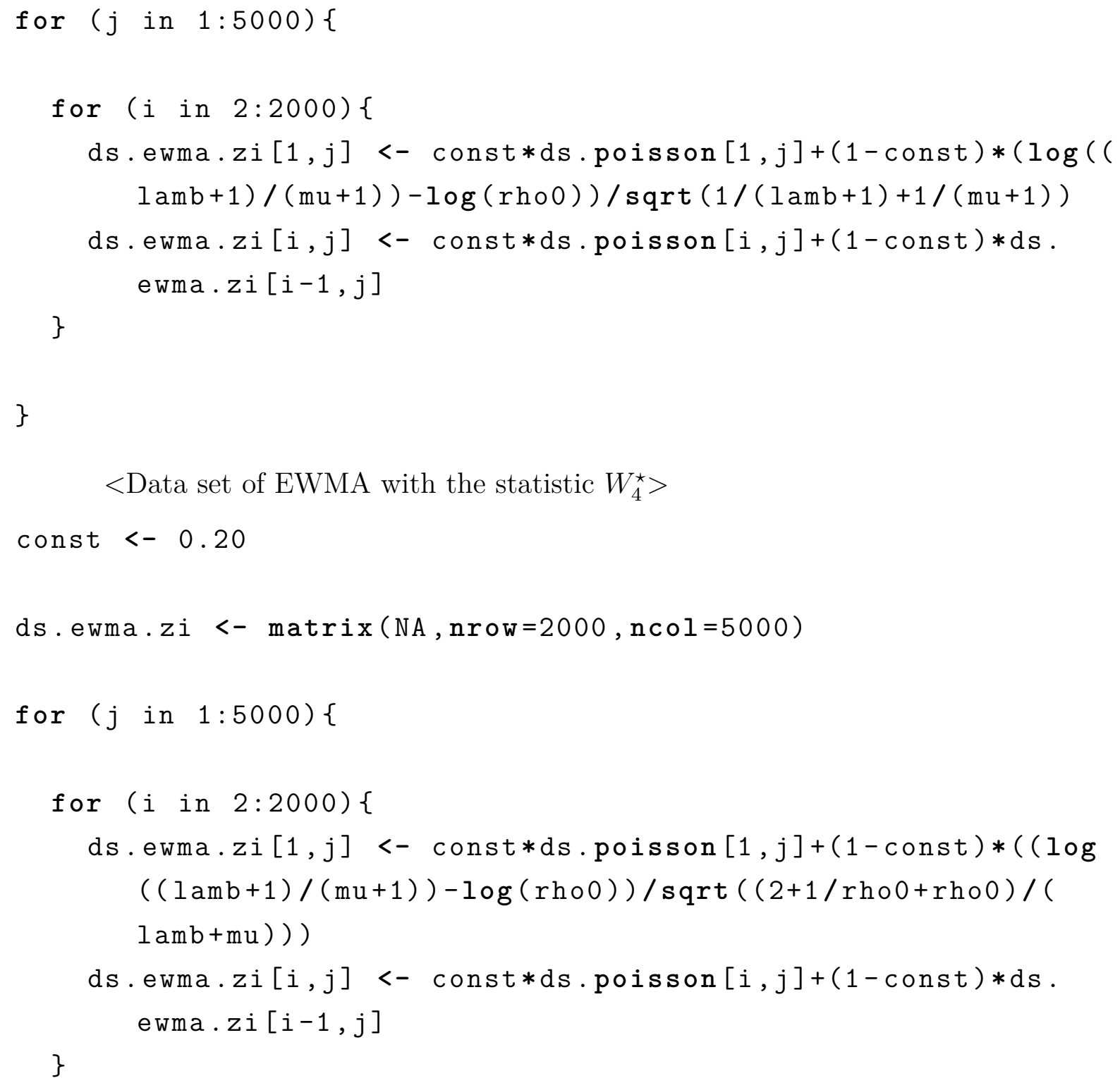




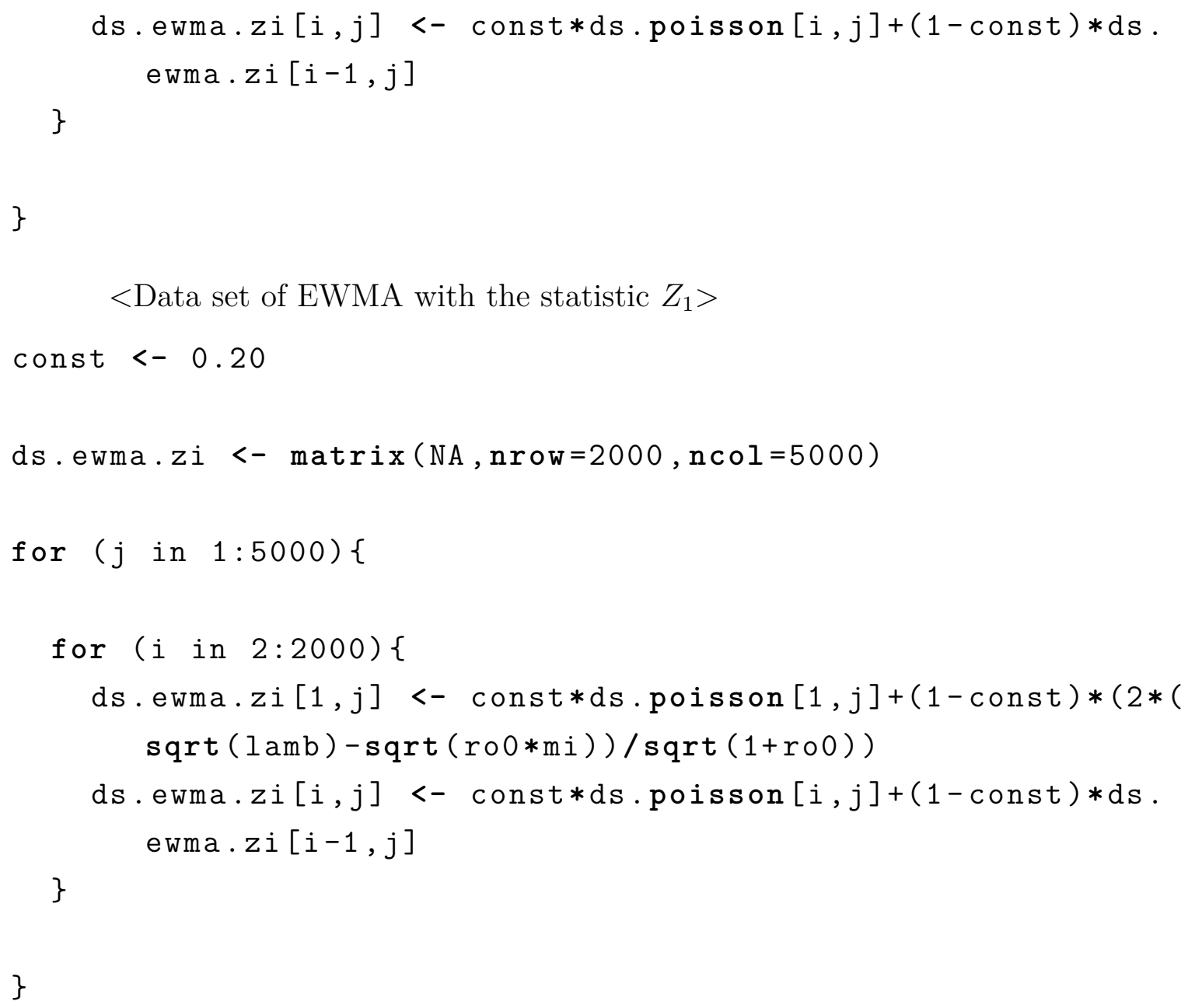

$<$ Data set of EWMA with the statistic $Z_{3}>$

const $<-0.20$ 


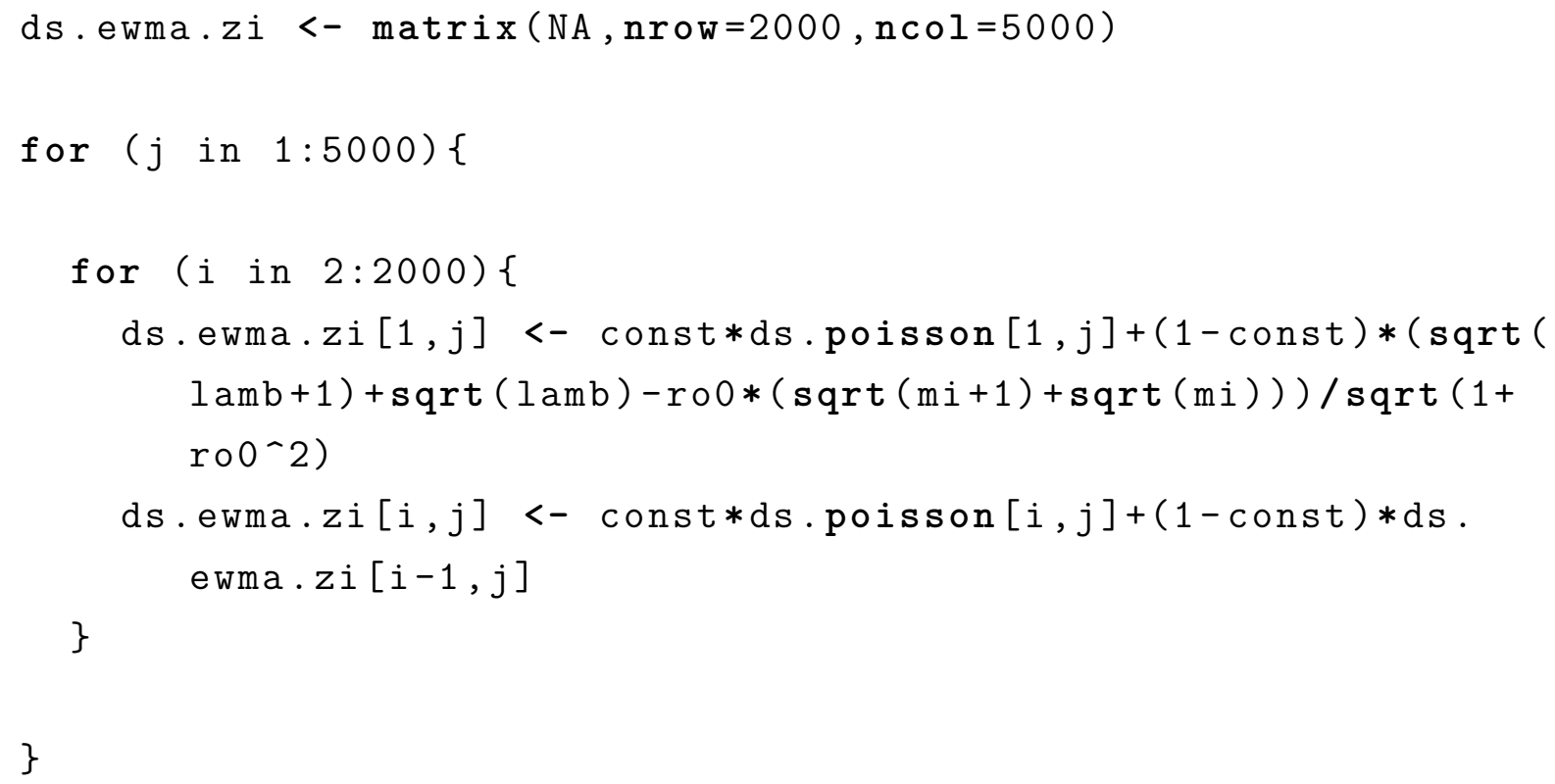

$<$ Data set of EWMA with the statistic $Z_{4}^{* *}>$

const $<-0.40$

ds. ewma.zi $<-\operatorname{matrix}(N A$, nrow $=2000$, ncol=5000)

for $(j$ in 1:5000)\{ 


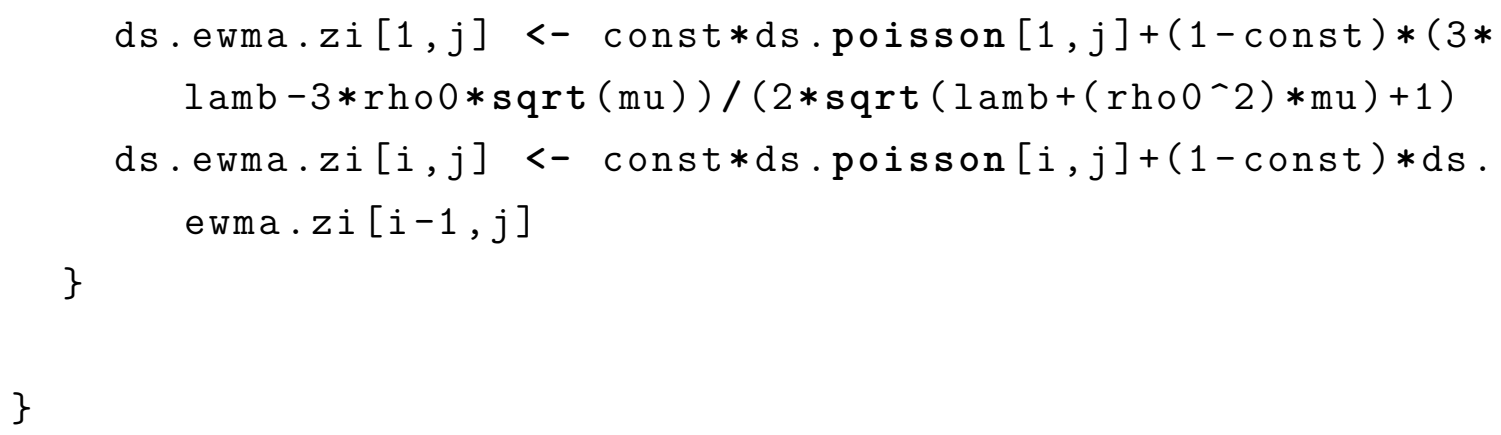

$<$ Data set of EWMA with the statistic $Z_{5}>$

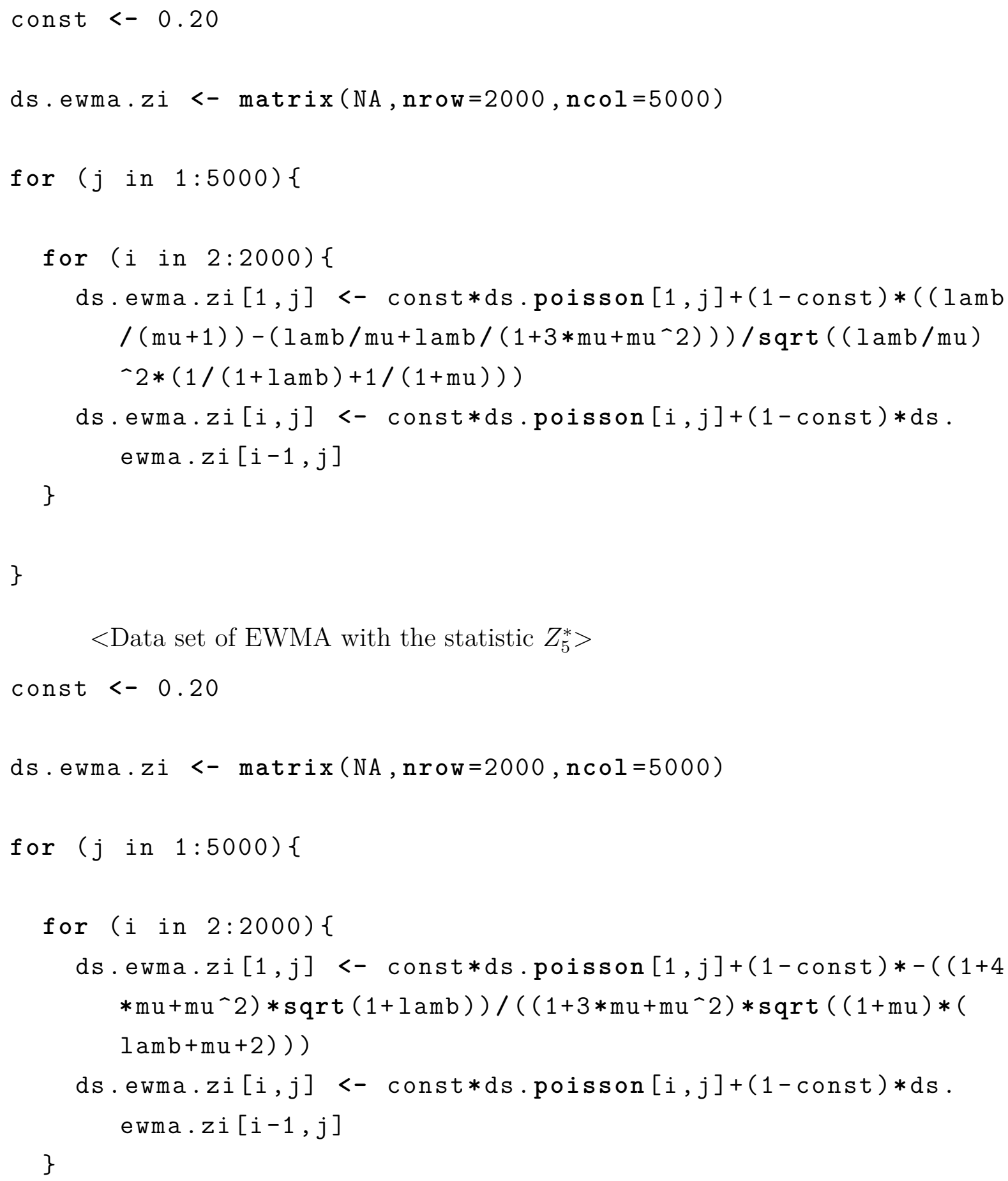


\}

$<$ ARL0 Estimation $>$

$\mathrm{UCL}<-0.9$

repeat \{

$\mathrm{UCL}<-\mathrm{UCL}+0.00001$

RL. TI $<-$ NULL

for $(j$ in $1: 5000)\{$

TI1 <- NULL

for ( $i$ in 1:2000)\{

TI1 [i] <- ds.poisson $[i, j]$

if ( TI 1 [i] > UCL) \{break\}

\}

RL.TI $[j]<-$ length (TI1)

\}

ARL $<-\operatorname{mean}($ RL.TI $)$

if $(A R L>170)\{$ break $\}$

\} 


\section{APPENDIX B - Source code of stage II}

$<$ ARL1 Estimation for Shewhart control chart in case $\lambda_{10}$ and $\lambda_{20}$ are known $>$

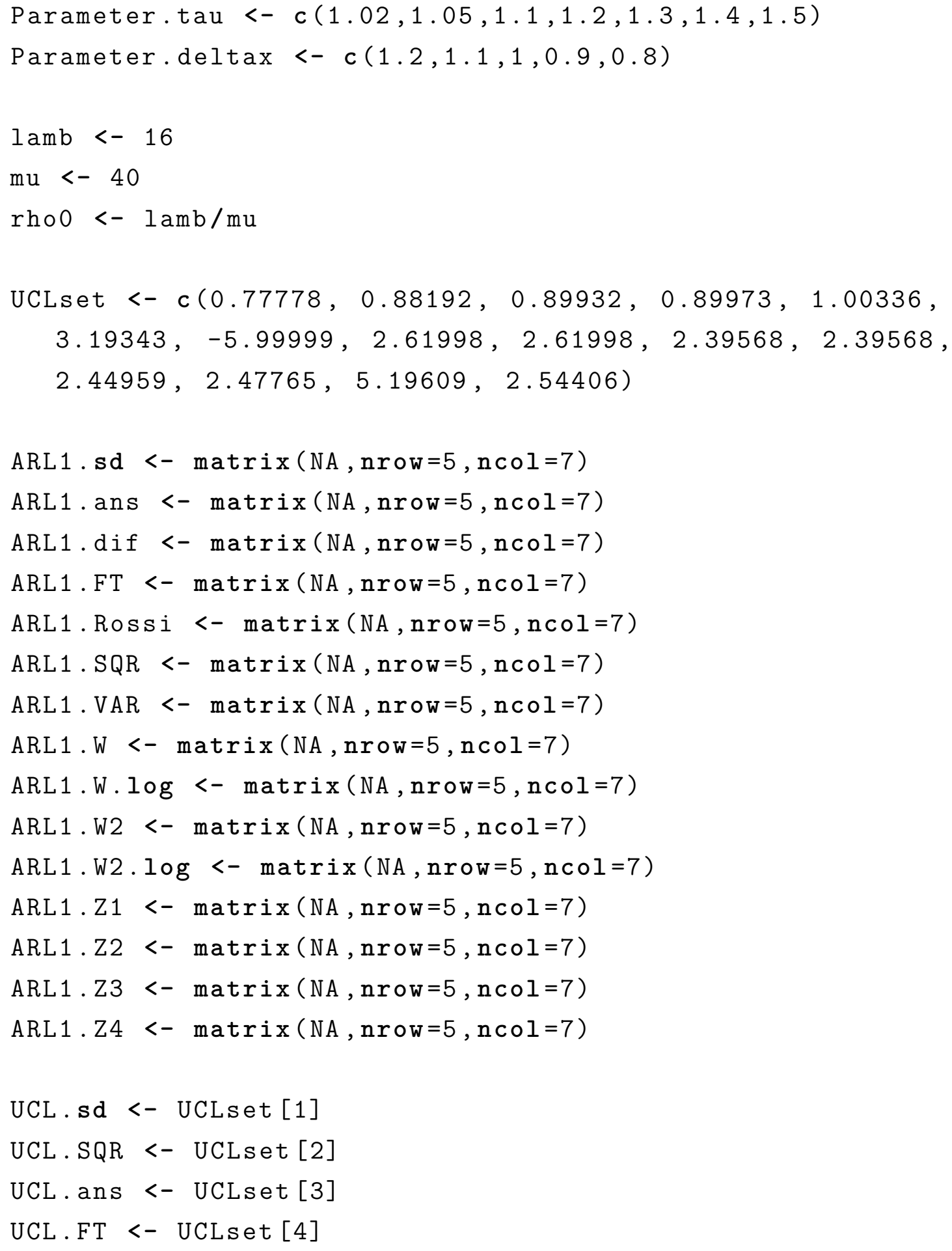




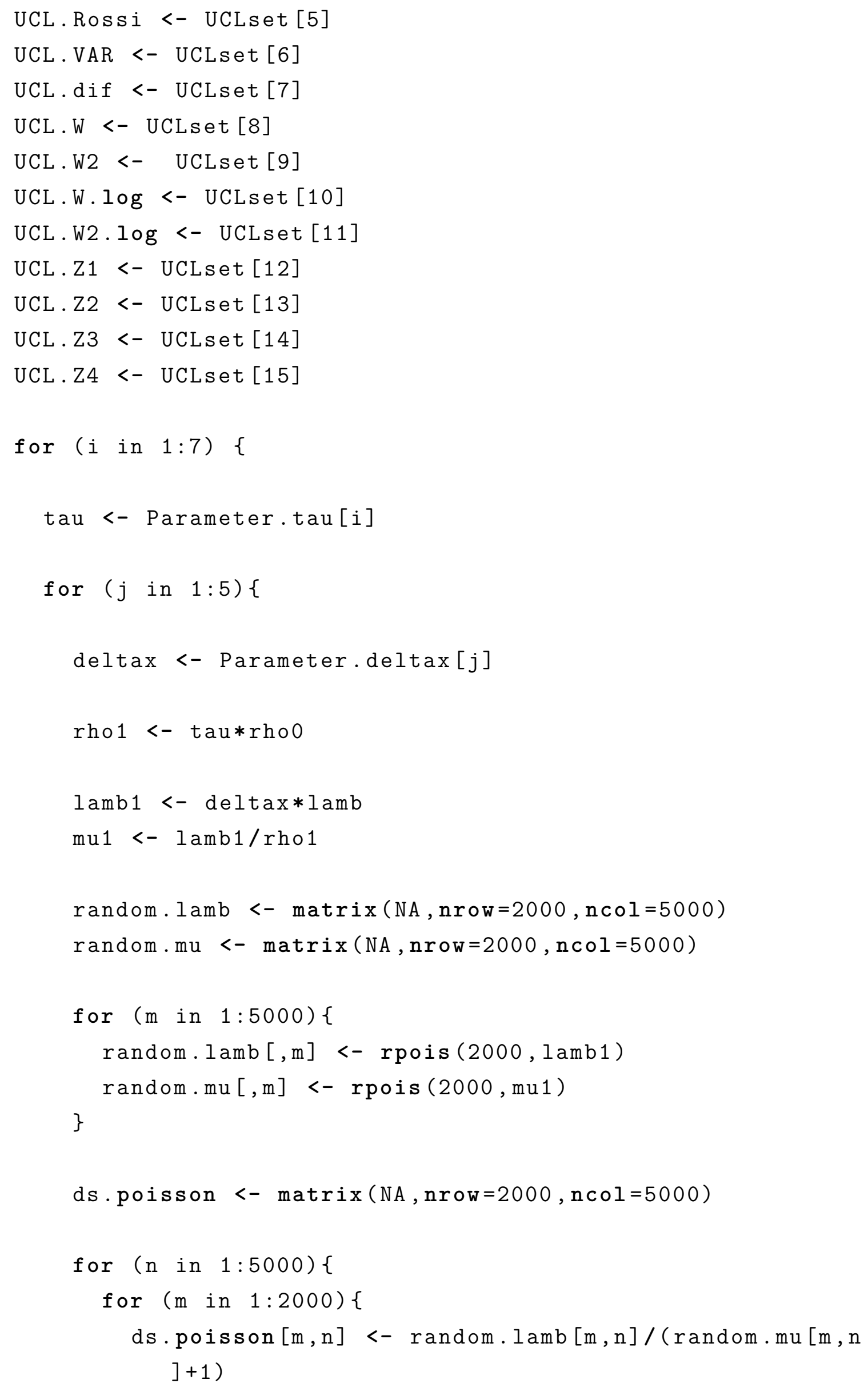




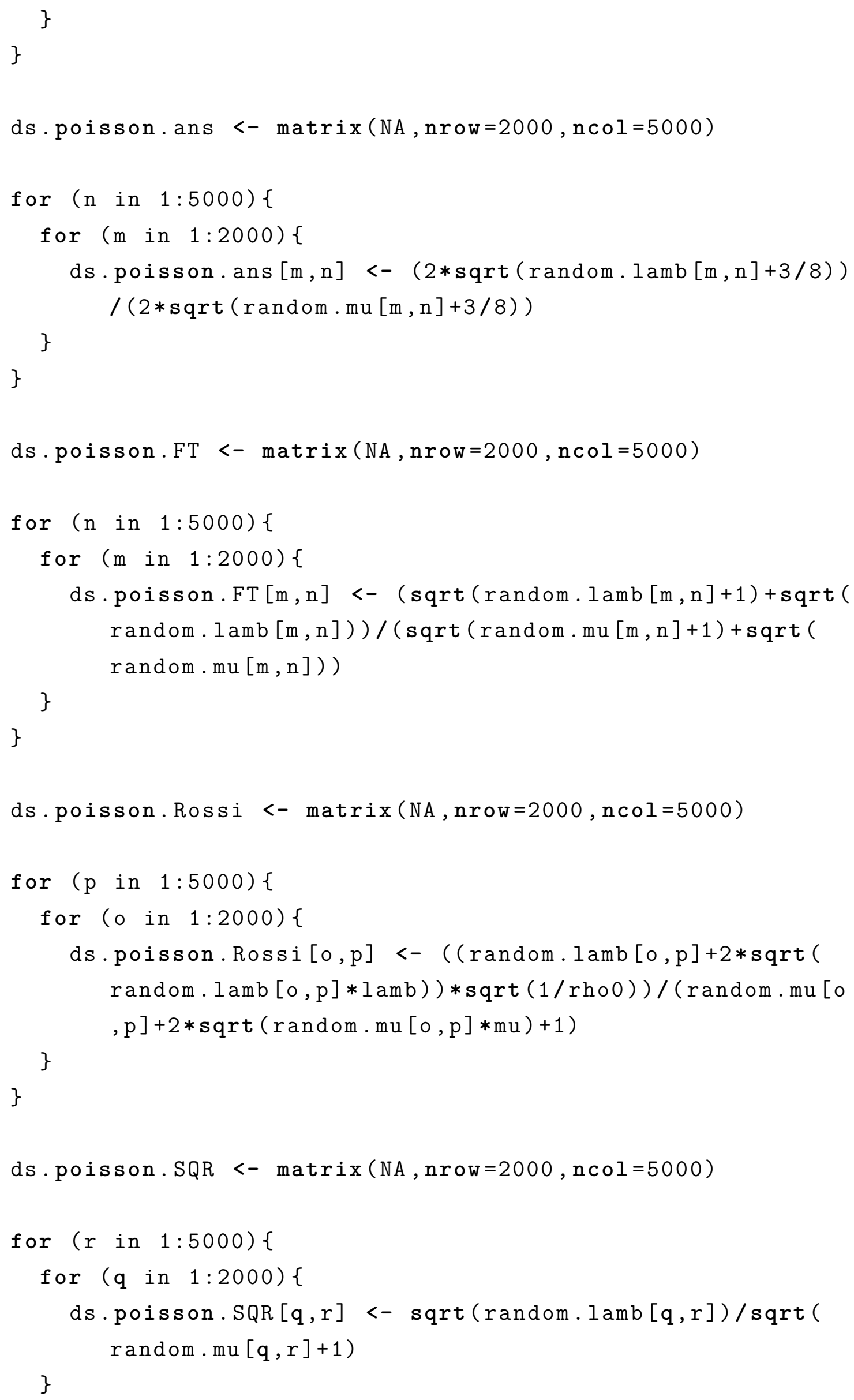


ds.poisson. VAR <- matrix (NA, nrow $=2000$, ncol=5000)

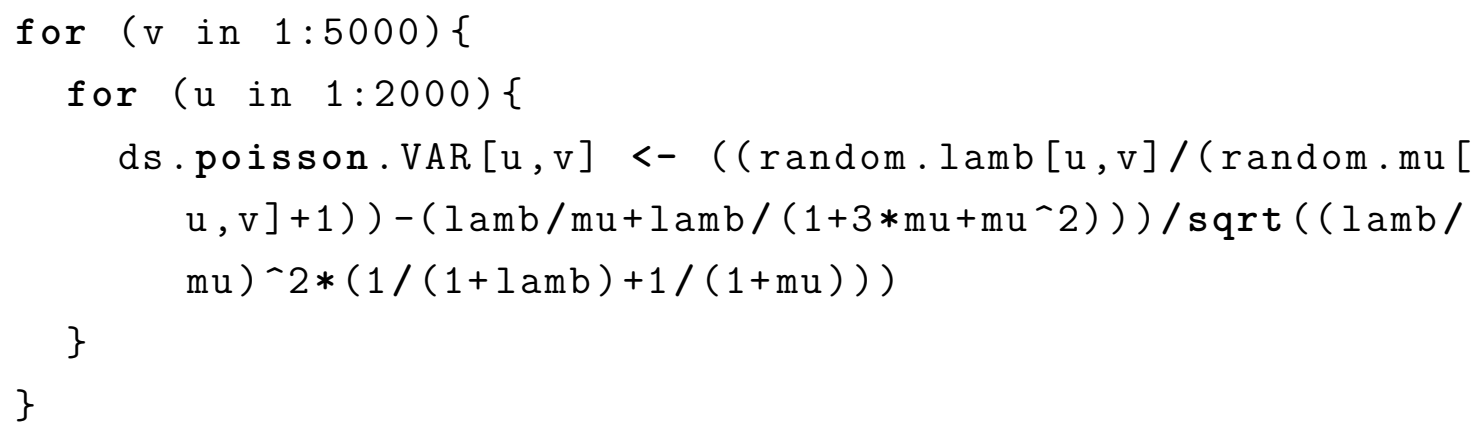




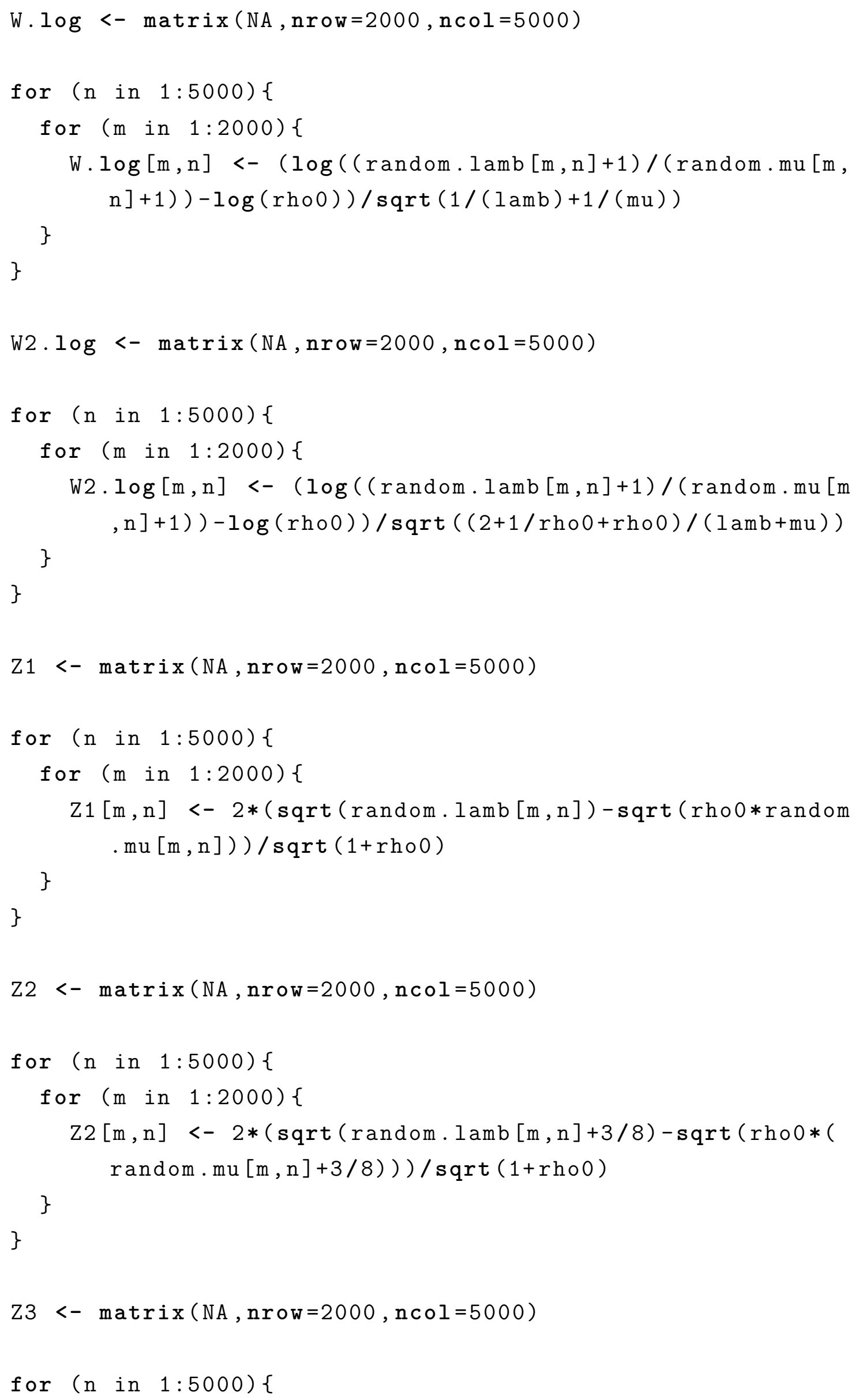




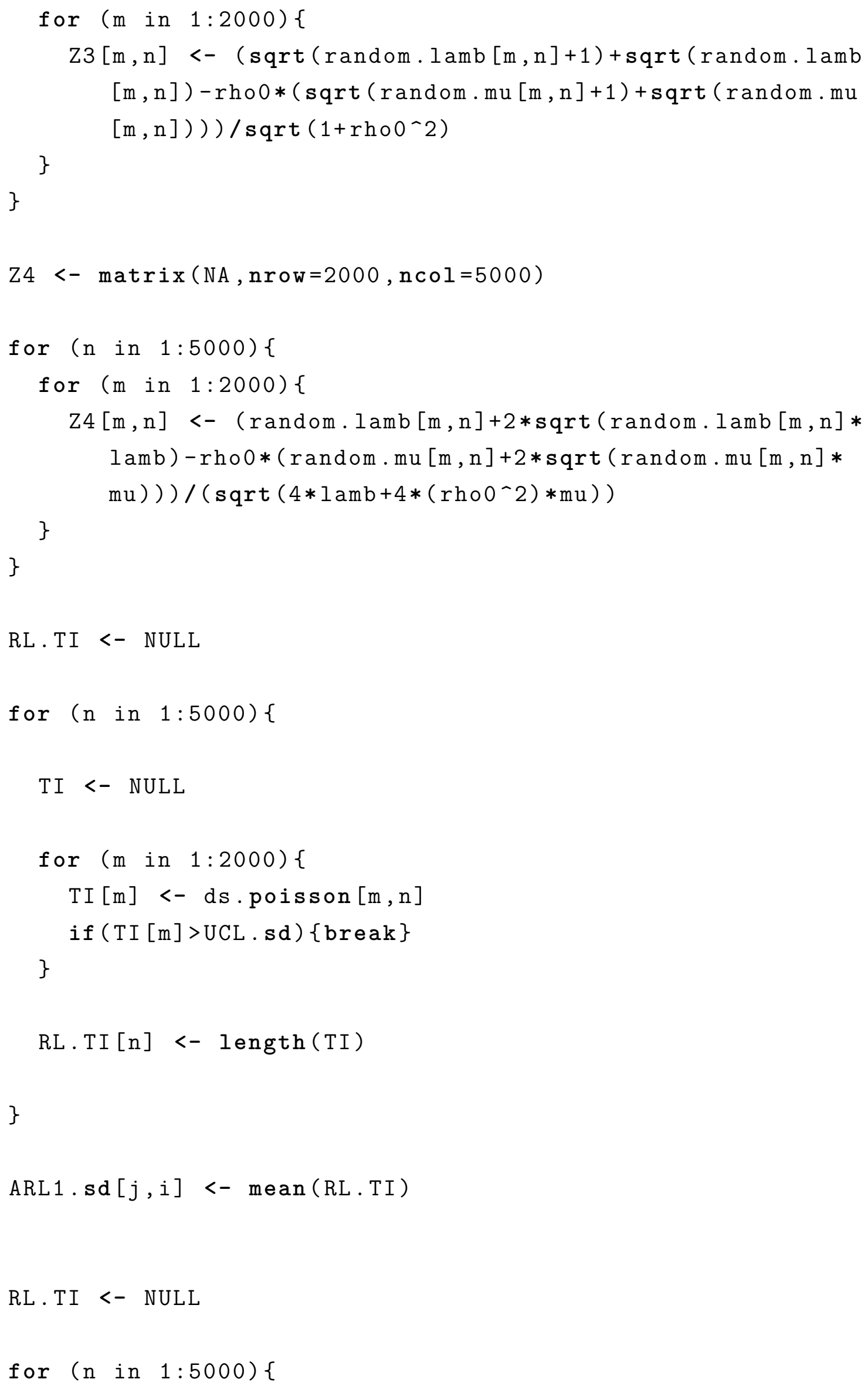




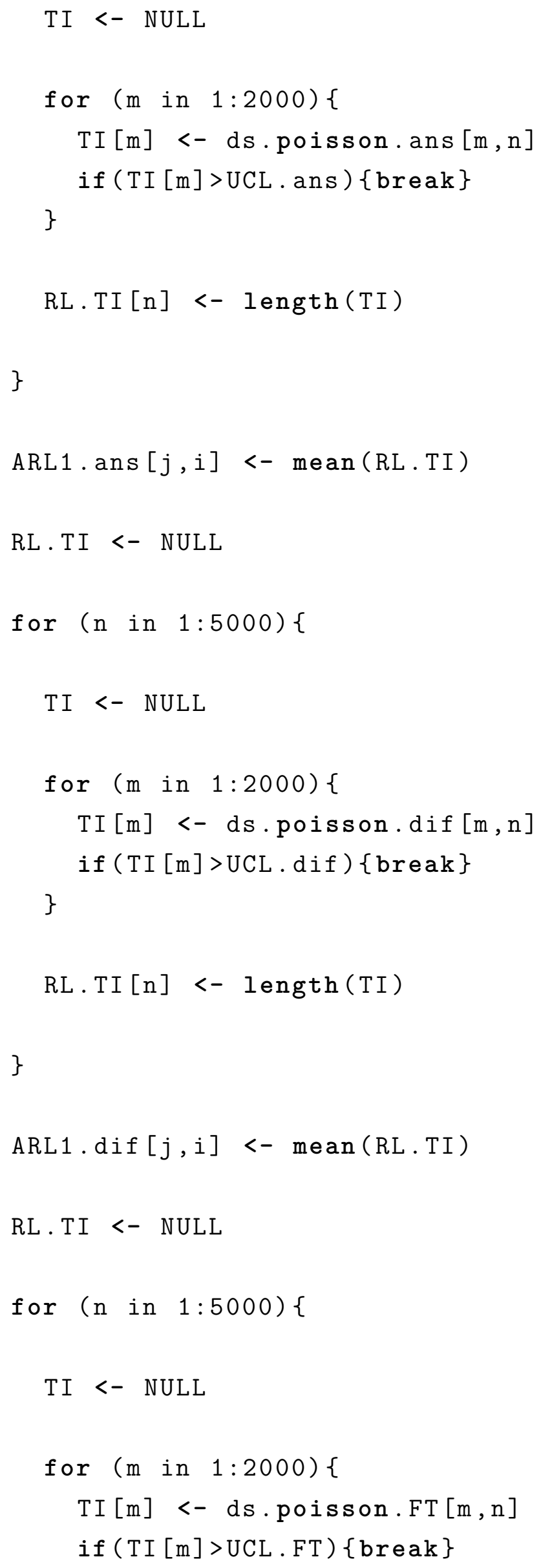




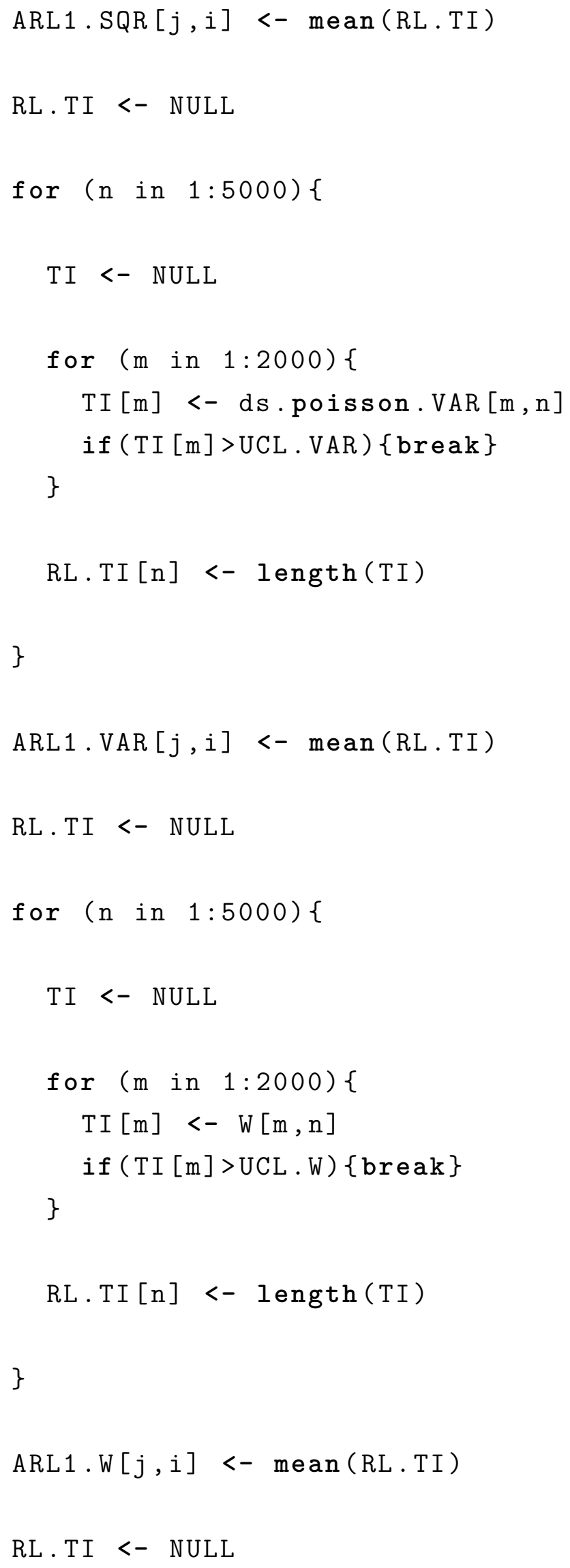




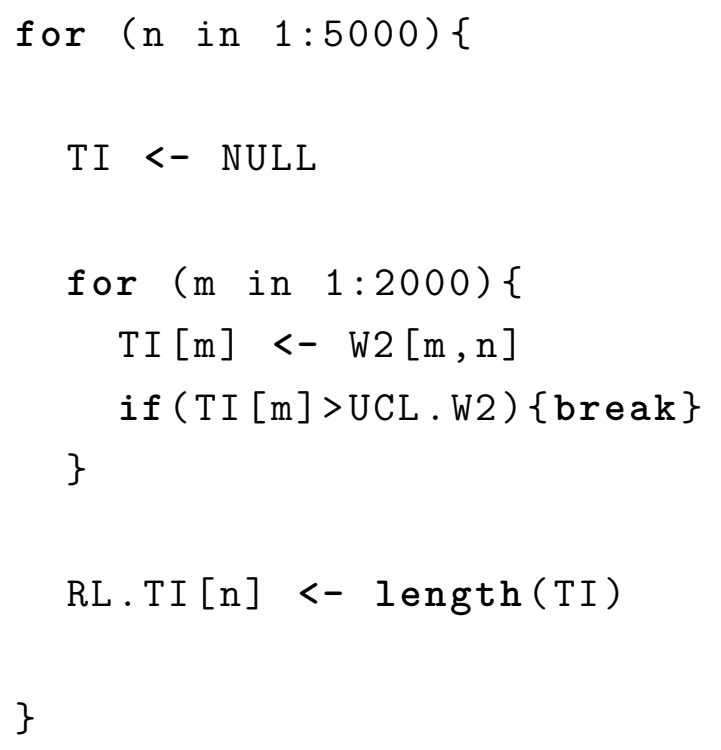




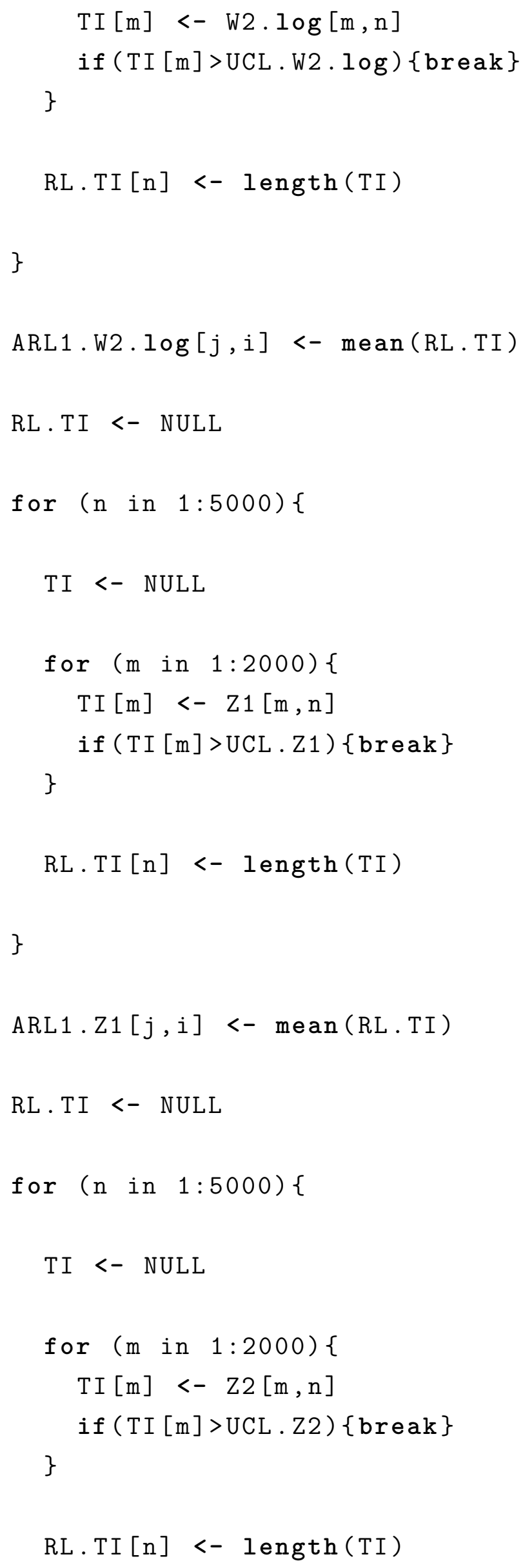


\}

ARL1.Z2 $[j, i]<-\operatorname{mean}(R L . T I)$

RL. TI $<-$ NULL

for ( $\mathrm{n}$ in 1:5000)\{

TI $<-$ NULL

for $(m$ in $1: 2000)\{$

TI $[\mathrm{m}]<-\mathrm{Z} 3[\mathrm{~m}, \mathrm{n}]$

if ( TI $[\mathrm{m}]>\mathrm{UCL}$. Z3) $\{$ break $\}$

\}

RL.TI $[n]<-$ length $(\mathrm{TI})$

\}

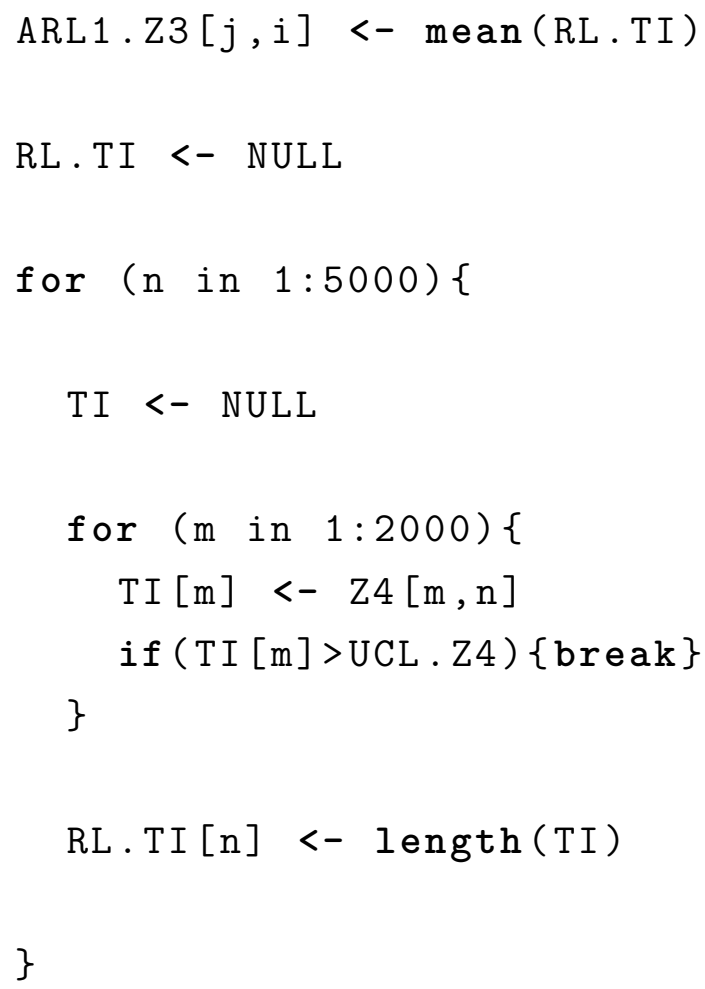


\}

ARL1.sd.ajt <- c(ARL1.sd [,1],ARL1.sd [,2], ARL1.sd [, 3], ARL1.sd $[, 4], A R L 1 . s d[, 5], A R L 1 . s d[, 6], A R L 1 . s d[, 7])$

ARL1 . ans . ajt <- c(ARL1 . ans [, 1], ARL1 . ans [, 2], ARL1 . ans [, 3], ARL1 . ans [ , 4], ARL1. ans [, 5], ARL1. ans [, 6], ARL1. ans [ , 7])

ARL1.dif.ajt <- c(ARL1.dif [,1],ARL1.dif [,2],ARL1.dif [,3],ARL1 .dif $[, 4], A R L 1 \cdot \operatorname{dif}[, 5], A R L 1 \cdot \operatorname{dif}[, 6], A R L 1 \cdot \operatorname{dif}[, 7])$

ARL1.FT.ajt $<-c(A R L 1 . F T[, 1], A R L 1 . F T[, 2], A R L 1 . F T[, 3], A R L 1 . F T$ $[, 4], \operatorname{ARL} 1 . \mathrm{FT}[, 5], \operatorname{ARL} 1 . \mathrm{FT}[, 6], \mathrm{ARL} 1 . \mathrm{FT}[, 7])$

ARL1.Rossi.ajt <- c(ARL1.Rossi[,1],ARL1.Rossi[,2], ARL1.Rossi [, 3], ARL1.Rossi [ , 4], ARL1.Rossi [ 5], ARL1.Rossi [ ,6] , ARL1. Rossi $[, 7]$ )

ARL1.SQR. ajt <- c(ARL1.SQR [, 1], ARL1.SQR [, 2], ARL1.SQR [, 3], ARL1 .SQR [ , 4], ARL1.SQR [ ,5], ARL1.SQR [ , 6], ARL1.SQR [ , 7])

ARL1.VAR . ajt <- c(ARL1.VAR [, 1], ARL1.VAR [, 2], ARL1.VAR [, 3], ARL1 .VAR [ , 4], ARL1.VAR [ , 5], ARL1.VAR [ , 6], ARL1.VAR [ , 7])

ARL1.W.ajt $<-c(A R L 1 . W[, 1], A R L 1 . W[, 2], A R L 1 . W[, 3], A R L 1 . W[, 4]$, ARL1.W $[, 5], A R L 1 . W[, 6], A R L 1 . W[, 7])$

ARL1.W2 . ajt <- c(ARL1.W2 [, 1], ARL1.W2 [, 2], ARL1.W2 [, 3], ARL1.W2 $[, 4], A R L 1$.W2 $[, 5], A R L 1 . W 2[, 6], A R L 1 . W 2[, 7])$

ARL1.W. $\log \cdot$ ajt $<-c(A R L 1 . W . \log [, 1], A R L 1 . W . \log [, 2], A R L 1 . W . \log$ [ , 3], ARL1.W. $\log [, 4], A R L 1 . W . \log [, 5], A R L 1 . W . \log [, 6], A R L 1 . W$. $\log [, 7])$

ARL1.W2.log.ajt <- c (ARL1.W2. $\log [, 1], A R L 1 . W 2 . \log [, 2], A R L 1 . W 2$. $\log [, 3], A R L 1 . W 2 . \log [, 4], A R L 1 . W 2 . \log [, 5], A R L 1 . W 2 . \log [, 6]$, ARL1.W2. $\log [, 7]$ )

ARL1.Z1.ajt <- c(ARL1.Z1 [, 1], ARL1.Z1 [, 2], ARL1.Z1 [, 3], ARL1.Z1 $[, 4], A R L 1 . Z 1[, 5], A R L 1 . Z 1[, 6], A R L 1 . Z 1[, 7])$ ARL1.Z2.ajt <- c(ARL1.Z2 [,1],ARL1.Z2 [, 2], ARL1.Z2 [, 3], ARL1.Z2 $[, 4], A R L 1 . Z 2[, 5], A R L 1 . Z 2[, 6], A R L 1 . Z 2[, 7])$ ARL1.Z3.ajt <- c(ARL1.Z3 [,1],ARL1.Z3 [, 2], ARL1.Z3 [, 3], ARL1.Z3 $[, 4], A R L 1 . Z 3[, 5], A R L 1 . Z 3[, 6], A R L 1 . Z 3[, 7])$ ARL1.Z4.ajt <- c(ARL1.Z4 [, 1], ARL1.Z4 [, 2], ARL1.Z4 [, 3], ARL1.Z4 $[, 4], A R L 1 . Z 4[, 5], A R L 1 . Z 4[, 6], A R L 1 . Z 4[, 7])$

Result <- matrix(c(ARL1.sd.ajt,ARL1.SQR.ajt,ARL1.ans.ajt, ARL1 .FT.ajt, ARL1.Rossi.ajt, ARL1.VAR.ajt, ARL1.dif .ajt, ARL1.W. 
ajt, ARL1.W2.ajt, ARL1.W. log.ajt, ARL1.W2.log.ajt, ARL1.Z1.ajt , ARL1.Z2 . ajt, ARL1.Z3 . ajt, ARL1.Z4. ajt), nrow=35, ncol=15, byrow $=$ FALSE)

$<$ ARL1 Estimation for Shewhart control chart in case $\lambda_{10}$ and $\lambda_{20}$ are unknown $>$

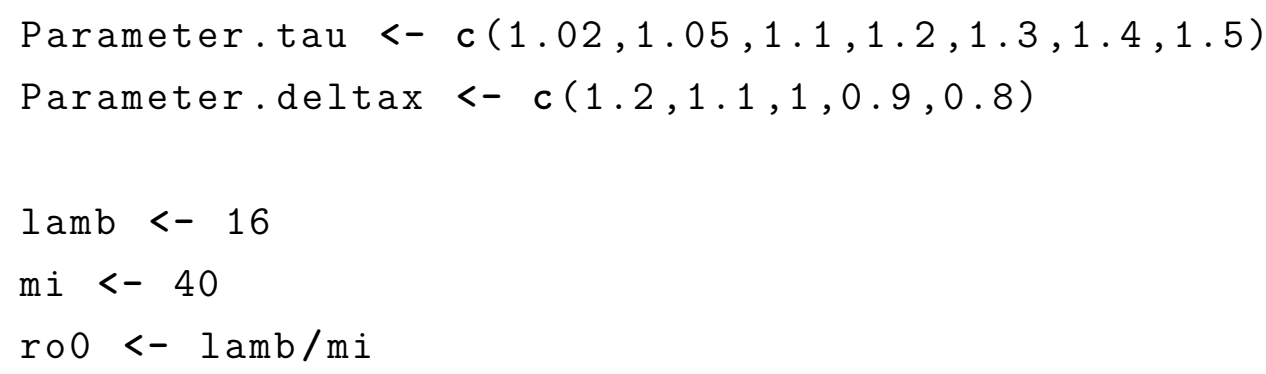




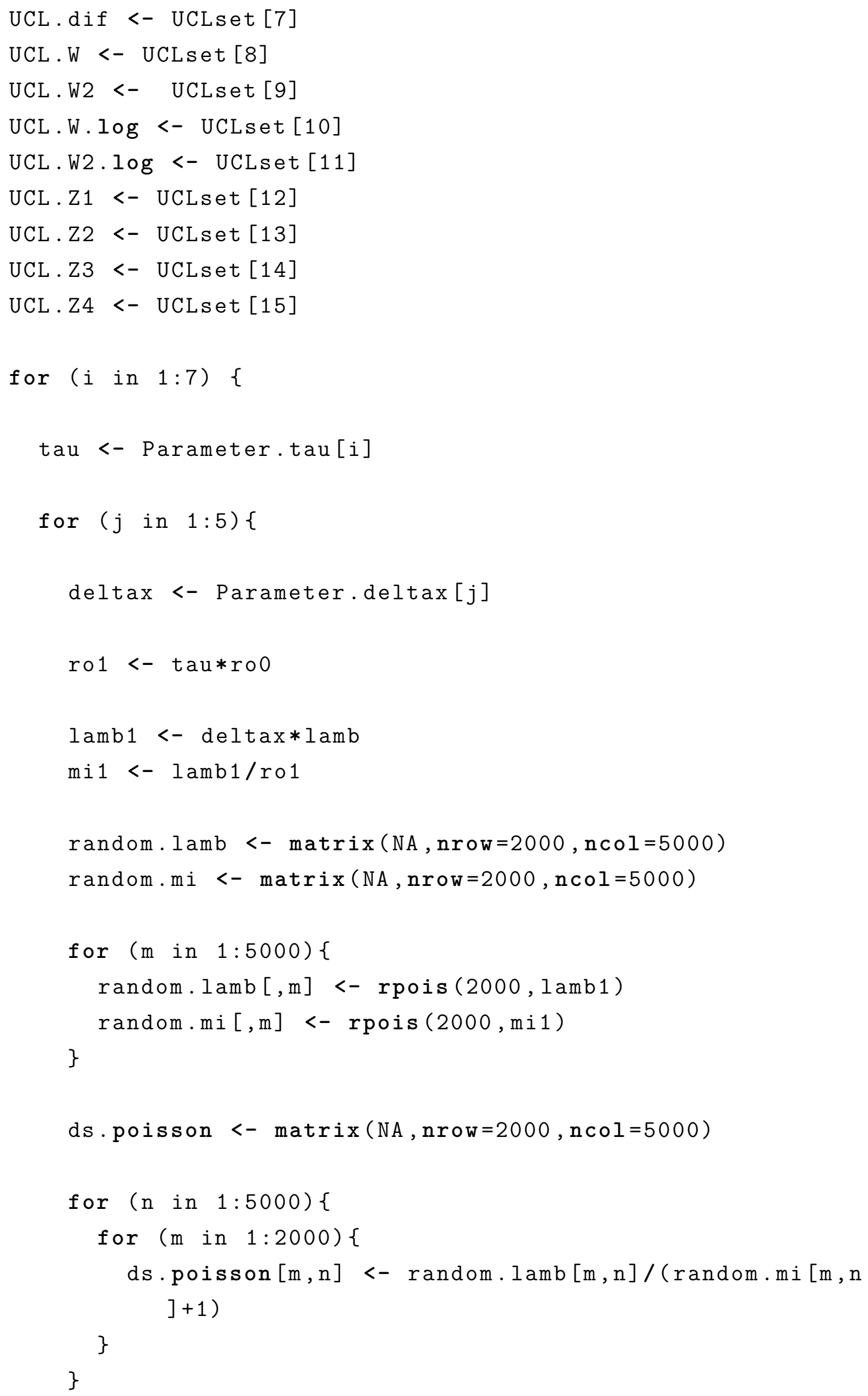




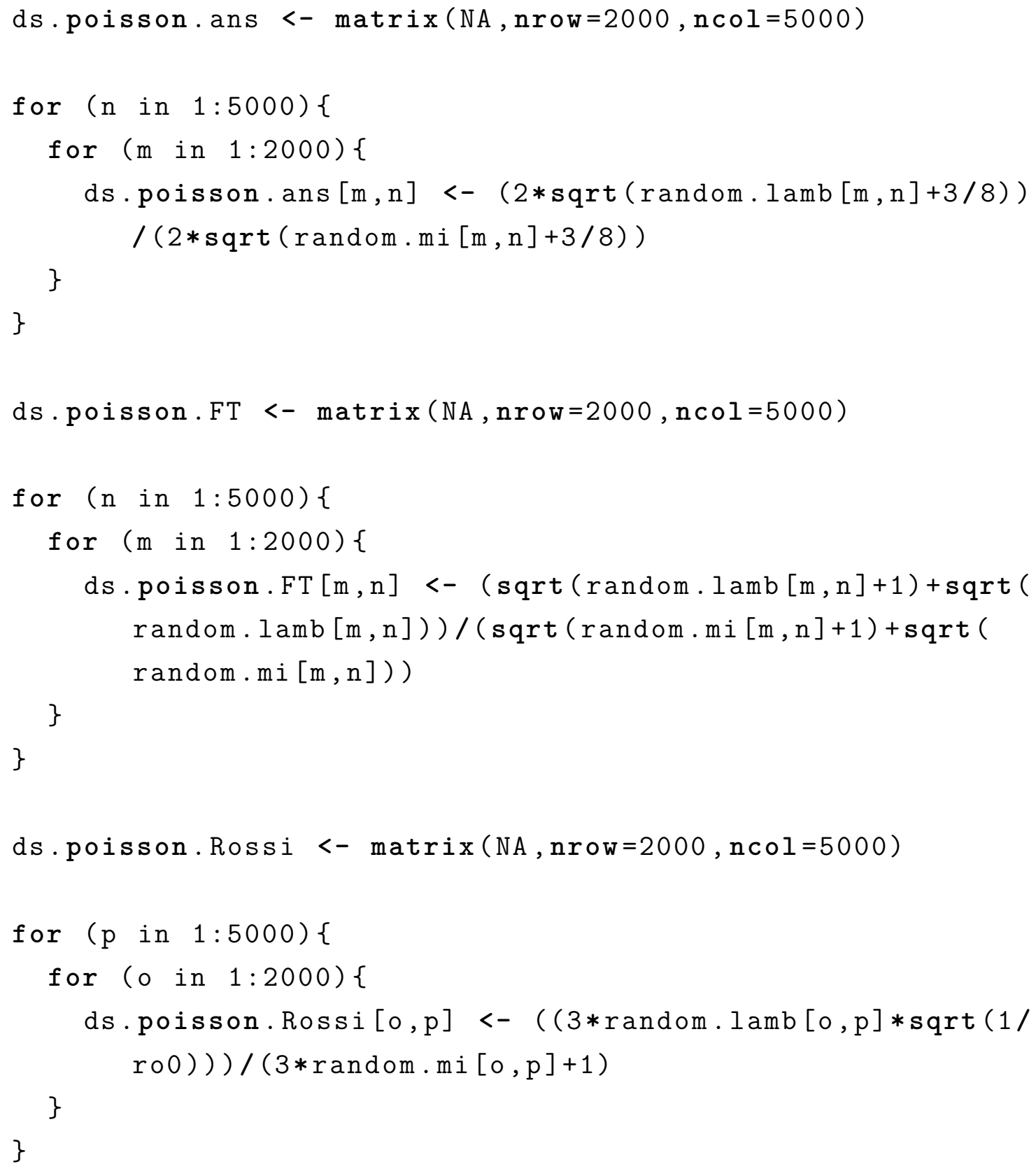

ds.poisson. VAR <- matrix $(N A$, nrow $=2000$, ncol $=5000)$ 


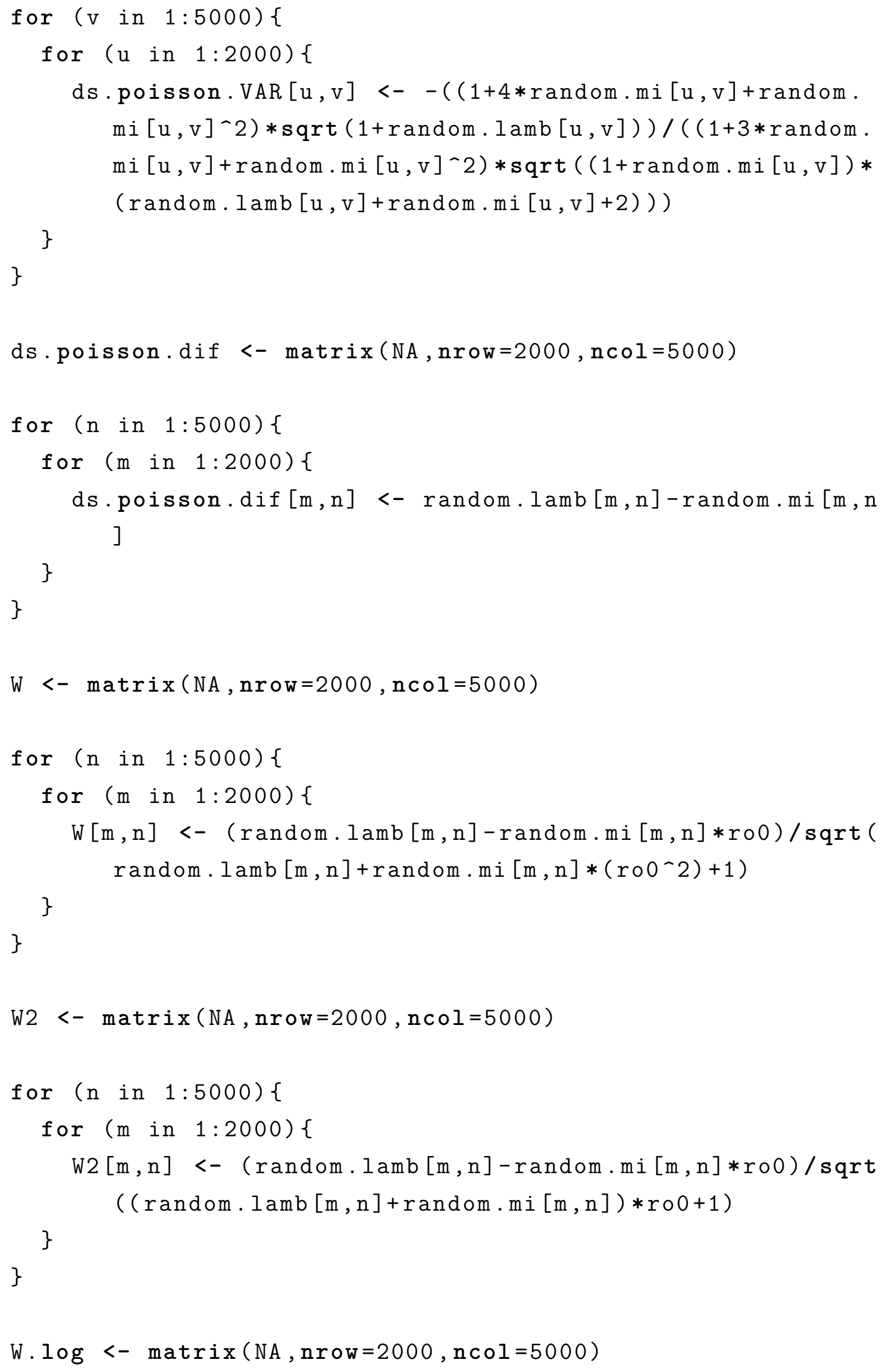




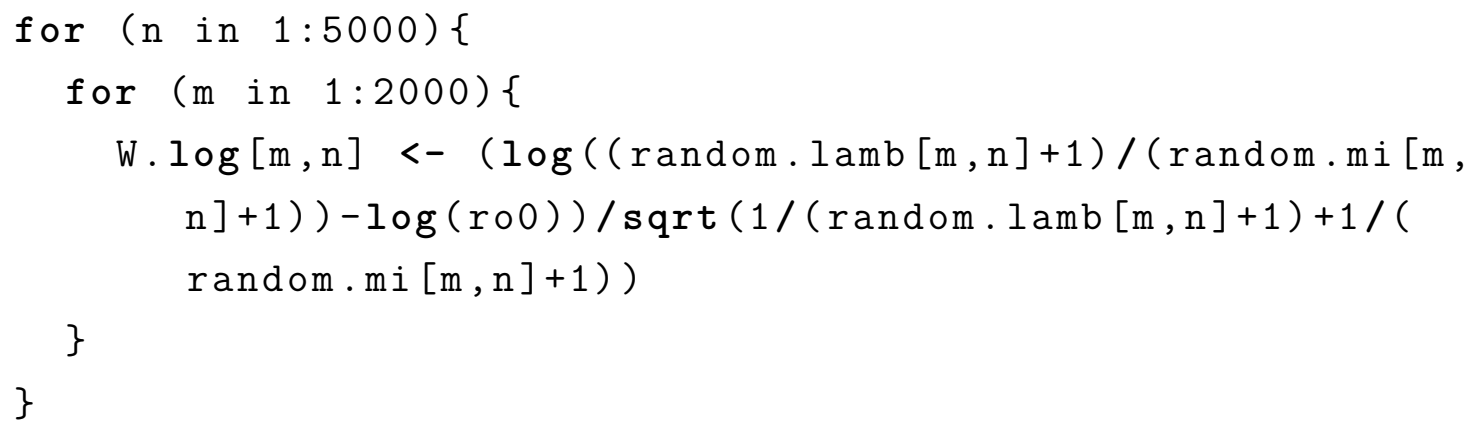




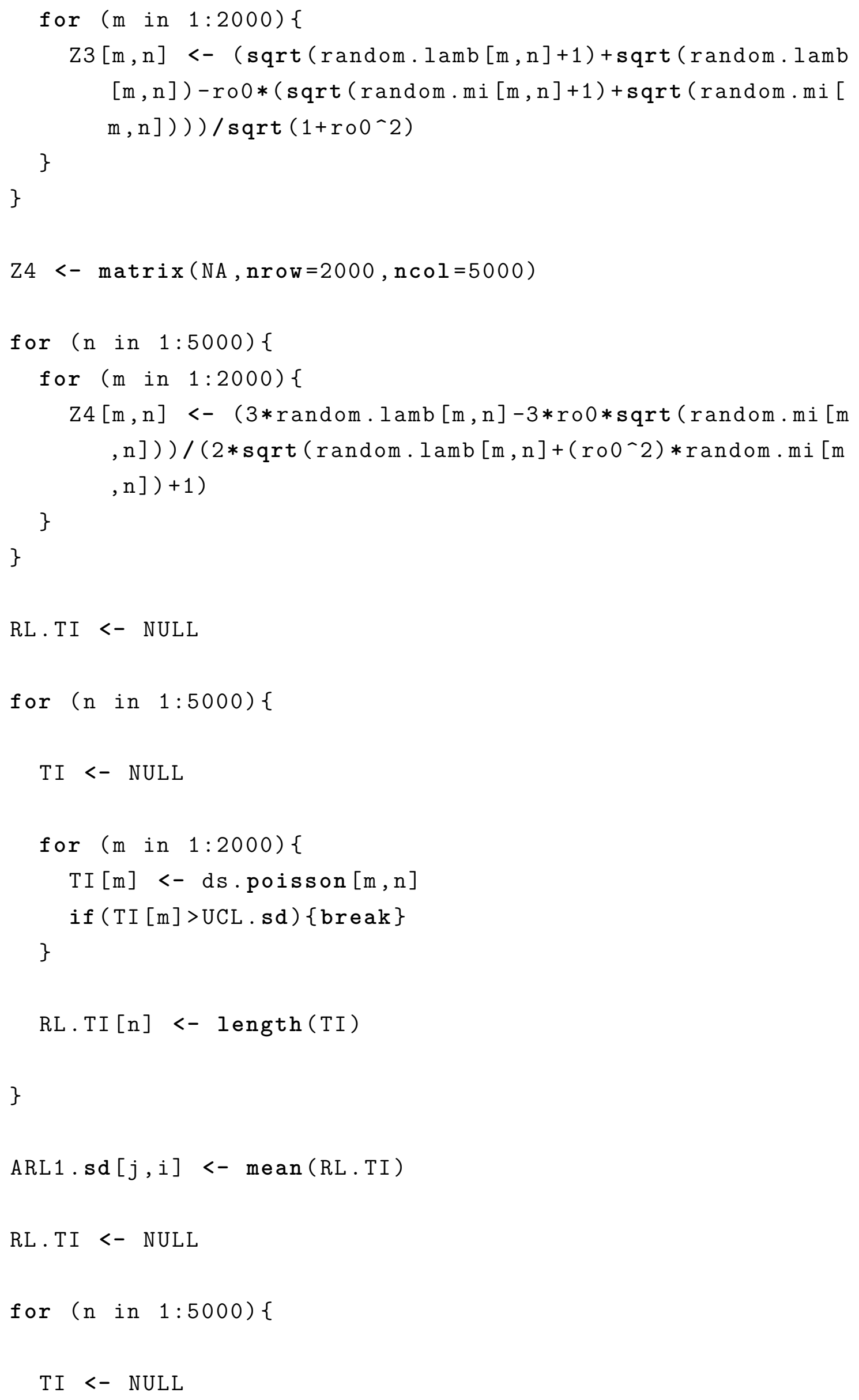




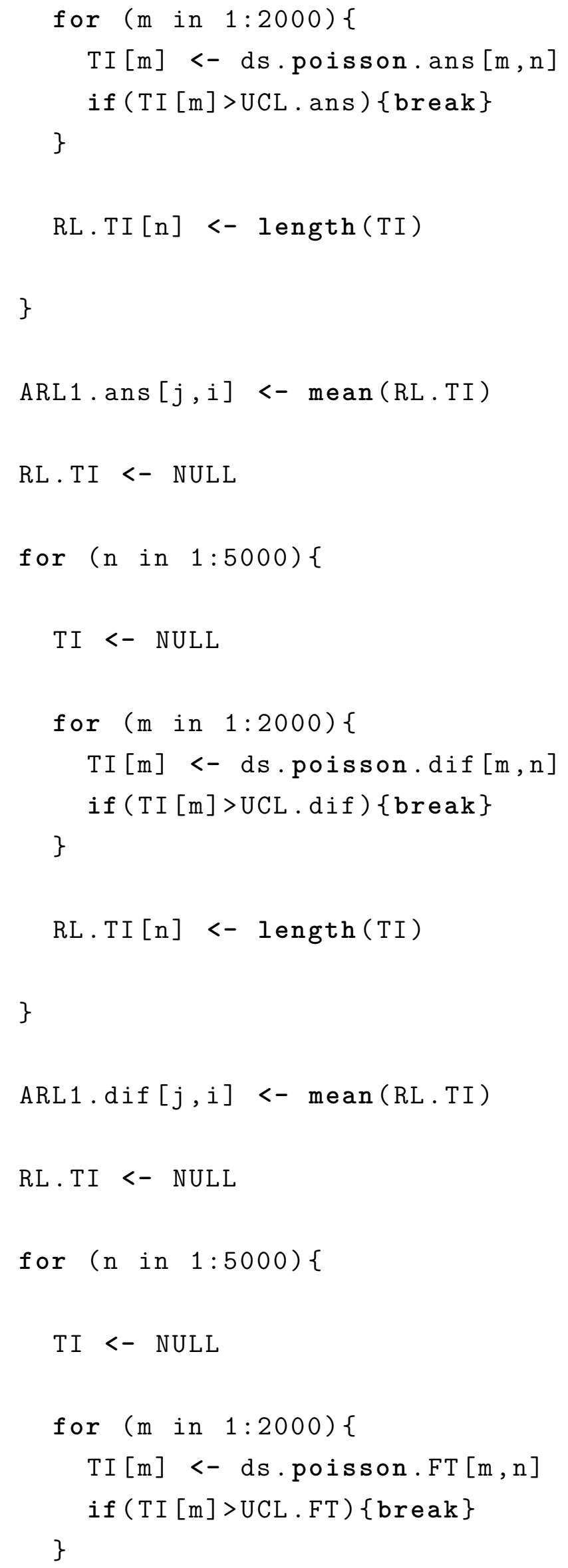




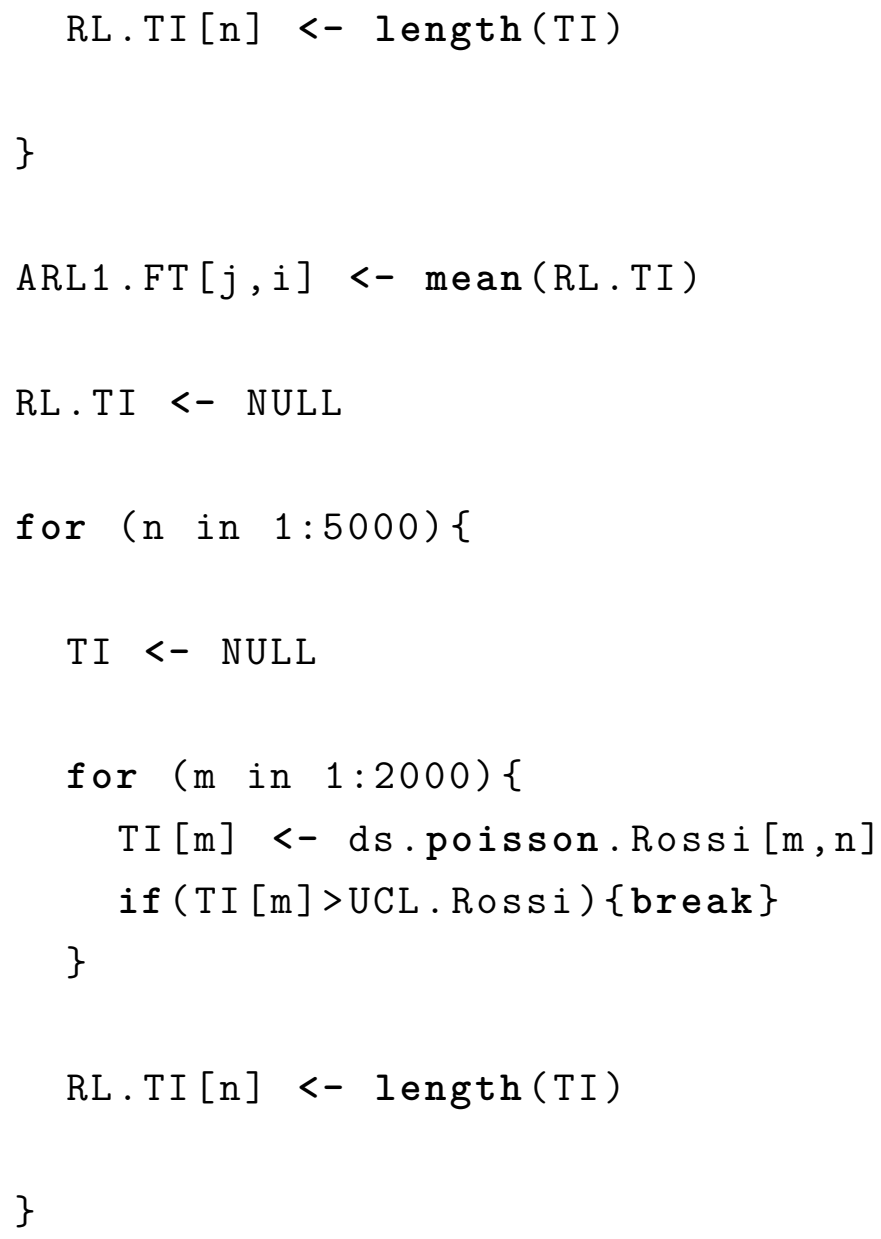




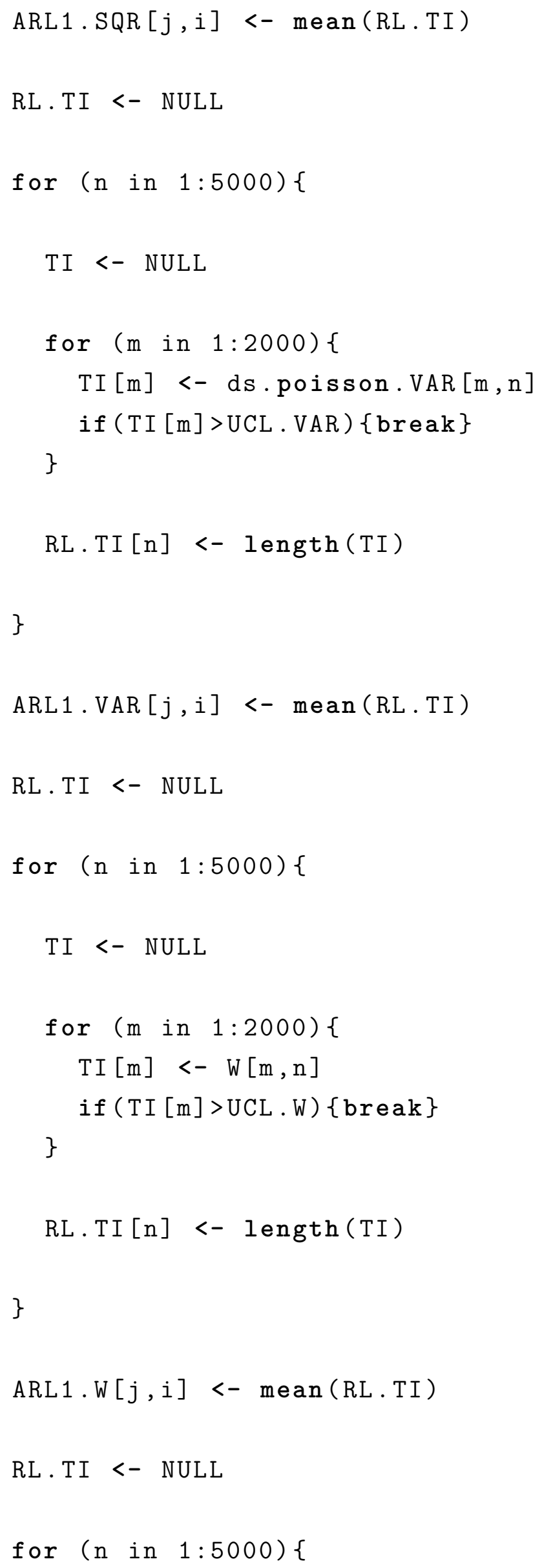




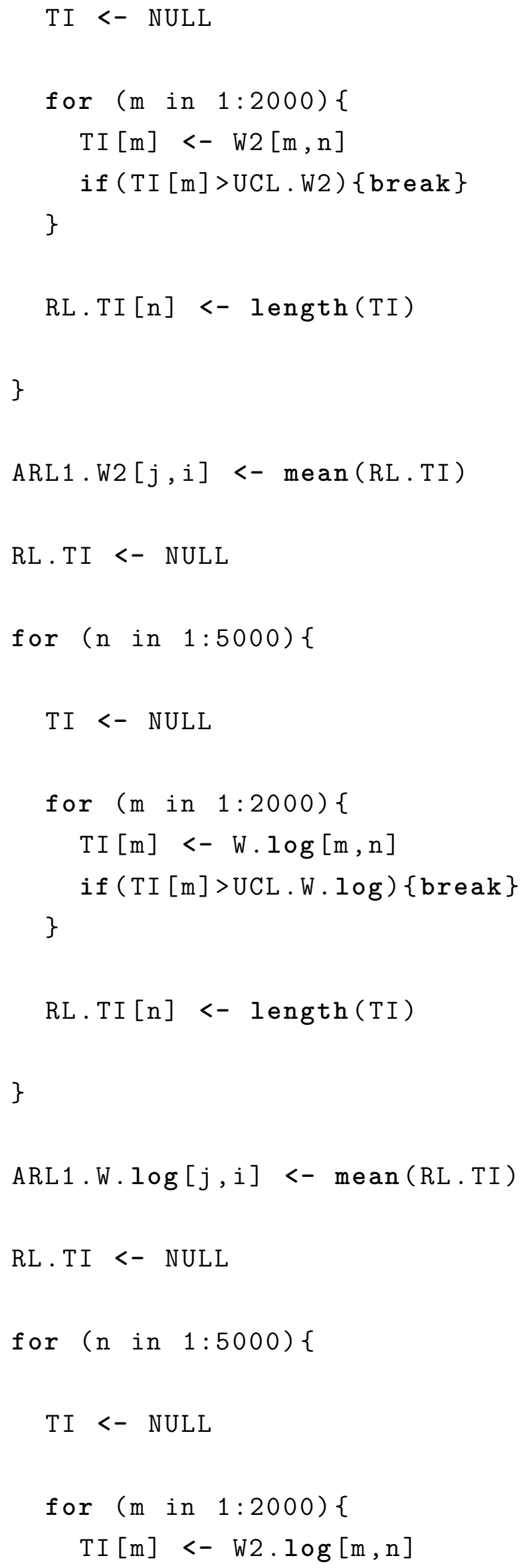




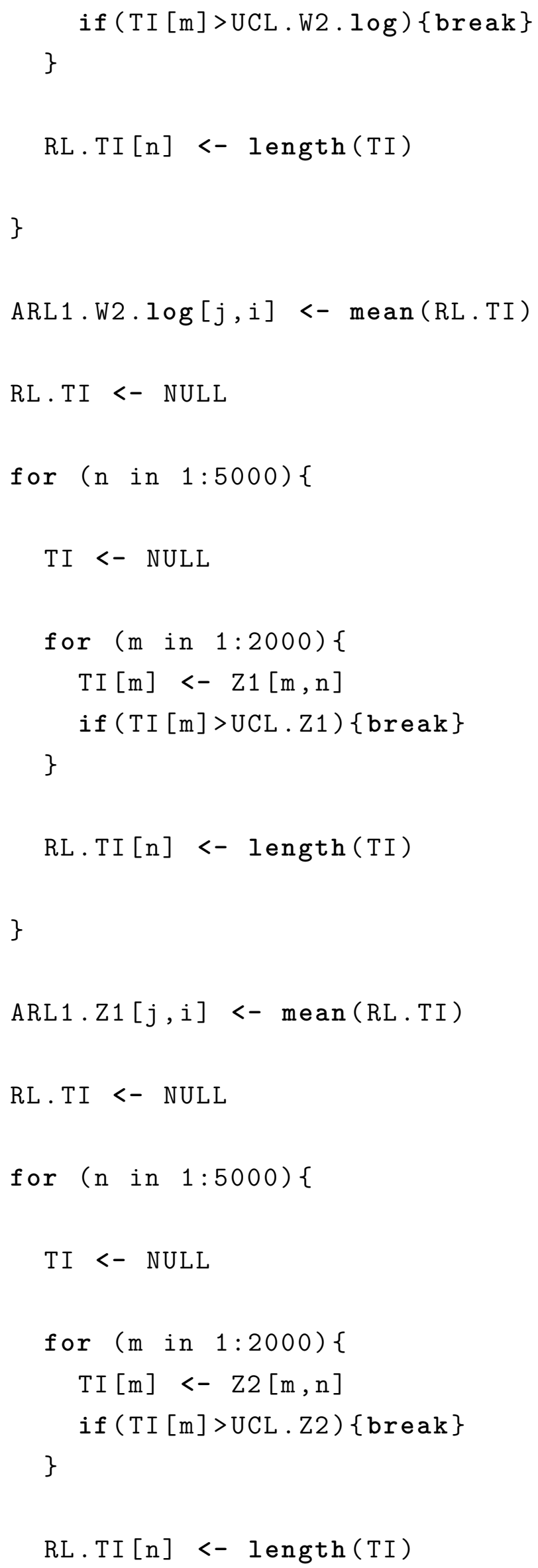




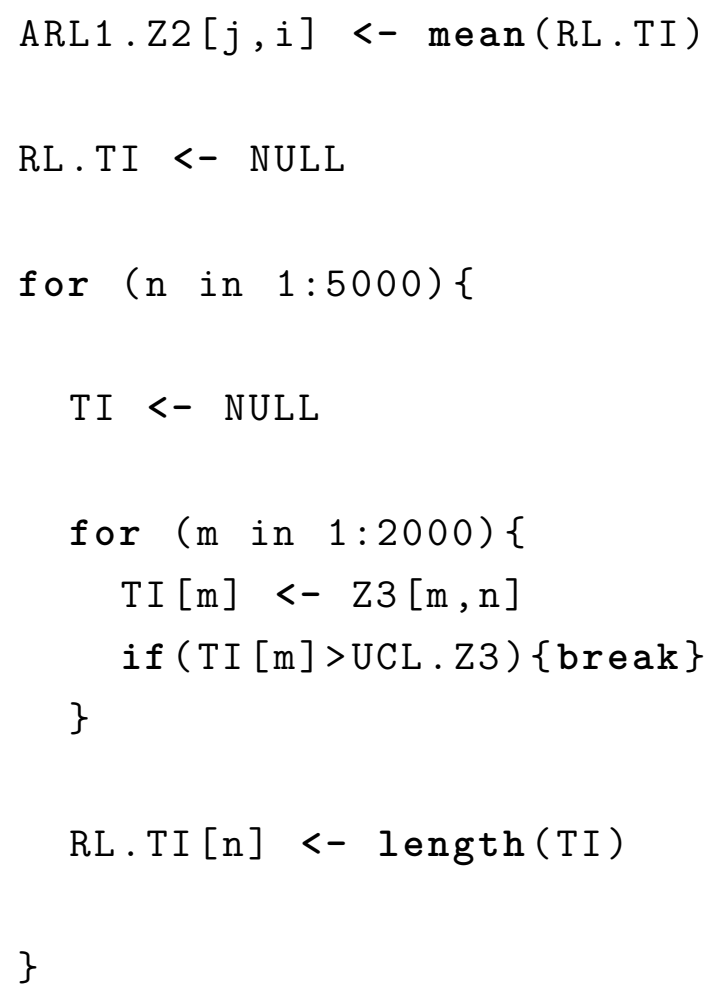


ARL1.sd.ajt <- c(ARL1.sd [,1],ARL1.sd [, 2], ARL1.sd [, 3], ARL1.sd $[, 4], A R L 1 . s d[, 5], A R L 1 . s d[, 6], A R L 1 . s d[, 7])$

ARL1. ans.ajt <- c(ARL1. ans [,1], ARL1. ans [, 2], ARL1. ans [, 3], ARL1 . ans [ , 4], ARL1. ans [ ,5], ARL1. ans [, 6], ARL1. ans [ , 7])

ARL1.dif.ajt <- c(ARL1.dif [,1],ARL1.dif [,2],ARL1.dif [,3],ARL1 .dif [, 4], ARL1.dif [,5], ARL1.dif [,6], ARL1.dif [, 7])

ARL1.FT.ajt <- c(ARL1.FT [, 1], ARL1.FT [, 2], ARL1.FT [, 3], ARL1.FT $[, 4], \operatorname{ARL} 1 . \mathrm{FT}[, 5], \operatorname{ARL} 1 . \mathrm{FT}[, 6], \operatorname{ARL} 1 . \mathrm{FT}[, 7])$

ARL1.Rossi.ajt <- c(ARL1.Rossi[,1],ARL1.Rossi [,2], ARL1.Rossi [, 3], ARL1.Rossi [ , 4], ARL1.Rossi [, 5], ARL1.Rossi [, 6], ARL1.

Rossi [, 7])

ARL1.SQR.ajt <- c (ARL1.SQR [, 1], ARL1.SQR [, 2], ARL1.SQR [, 3], ARL1 .SQR [ , 4], ARL1.SQR [ ,5], ARL1.SQR [, 6], ARL1.SQR [ , 7])

ARL1.VAR . ajt <- c(ARL1.VAR [, 1], ARL1.VAR [, 2], ARL1.VAR [, 3], ARL1 .VAR [ , 4], ARL1.VAR [ , 5], ARL1.VAR [ , 6], ARL1.VAR [ , 7])

ARL1.W.ajt <- c(ARL1.W[, 1], ARL1.W [, 2], ARL1.W [, 3], ARL1.W [, 4], ARL1.W[ 5], ARL1.W[, 6], ARL1.W [ , 7])

ARL1.W2.ajt <- c(ARL1.W2 [, 1], ARL1.W2 [, 2], ARL1.W2 [, 3], ARL1.W2 $[, 4], A R L 1$.W2 [ , 5], ARL1.W2 [, 6], ARL1.W2 [ , 7])

ARL1.W. $\log \cdot a j t<-c(A R L 1 . W . \log [, 1], A R L 1 . W . \log [, 2], A R L 1 . W . \log$ $[, 3], A R L 1 . W . \log [, 4], A R L 1 . W . \log [, 5], A R L 1 . W . \log [, 6], A R L 1 . W$. $\log [, 7])$

ARL1.W2. $\log \cdot$ ajt $<-c(A R L 1 . W 2 . \log [, 1], A R L 1 . W 2 . \log [, 2], A R L 1 . W 2$. $\log [, 3], A R L 1 . W 2 . \log [, 4], A R L 1 . W 2 . \log [, 5], A R L 1 . W 2 . \log [, 6]$, ARL1.W2. $\log [, 7])$

ARL1.Z1.ajt <- c(ARL1.Z1 [, 1],ARL1.Z1 [, 2], ARL1.Z1 [, 3], ARL1.Z1

$[, 4], A R L 1 . Z 1[, 5], A R L 1 . Z 1[, 6], A R L 1 . Z 1[, 7])$

ARL1.Z2.ajt <- c(ARL1.Z2 [, 1],ARL1.Z2 [, 2], ARL1.Z2 [, 3], ARL1.Z2

$[, 4], A R L 1 . Z 2[, 5], A R L 1 . Z 2[, 6], A R L 1 . Z 2[, 7])$

ARL1.Z3.ajt <- c(ARL1.Z3 [, 1],ARL1.Z3 [, 2], ARL1.Z3 [, 3], ARL1.Z3

$[, 4], A R L 1 . Z 3[, 5], A R L 1 . Z 3[, 6], A R L 1 . Z 3[, 7])$

ARL1.Z4.ajt <- c(ARL1.Z4 [, 1],ARL1.Z4 [, 2], ARL1.Z4 [, 3], ARL1.Z4

$[, 4], A R L 1 . Z 4[, 5], A R L 1 . Z 4[, 6], A R L 1 . Z 4[, 7])$

Result<- matrix(c(ARL1.sd.ajt,ARL1.SQR.ajt,ARL1.ans.ajt, ARL1 .FT.ajt, ARL1.Rossi.ajt, ARL1.VAR.ajt, ARL1.dif.ajt, ARL1.W. ajt, ARL1.W2.ajt, ARL1.W. log.ajt, ARL1.W2.log.ajt, ARL1.Z1.ajt 
, ARL1.Z2.ajt, ARL1.Z3. ajt, ARL1.Z4. ajt), nrow=35, ncol=15, byrow $=$ FALSE)

$<$ ARL1 Estimation for EWMA-type control chart in case $\lambda_{10}$ and $\lambda_{20}$ are known $>$

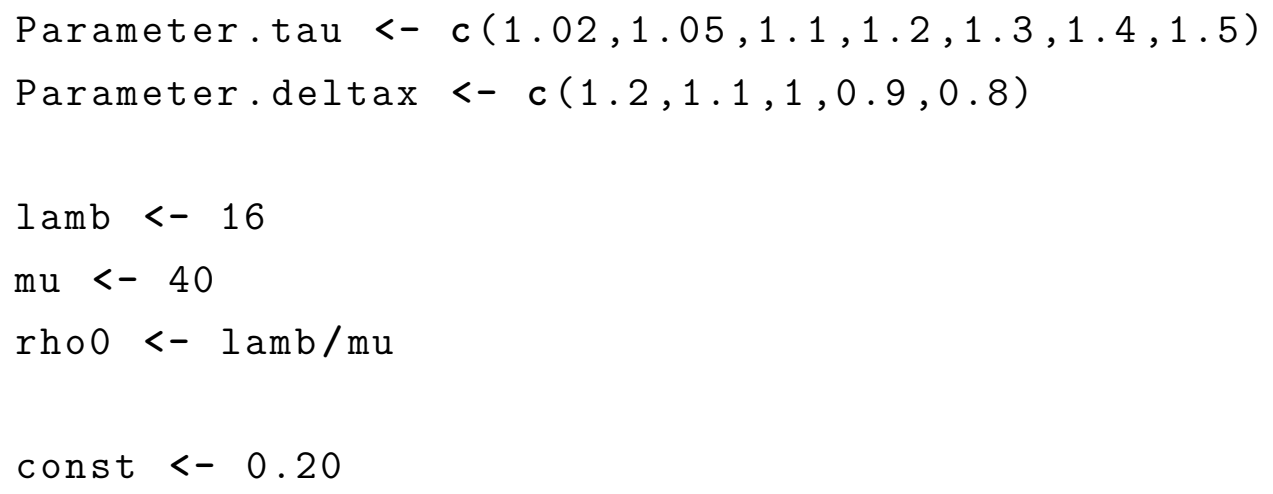




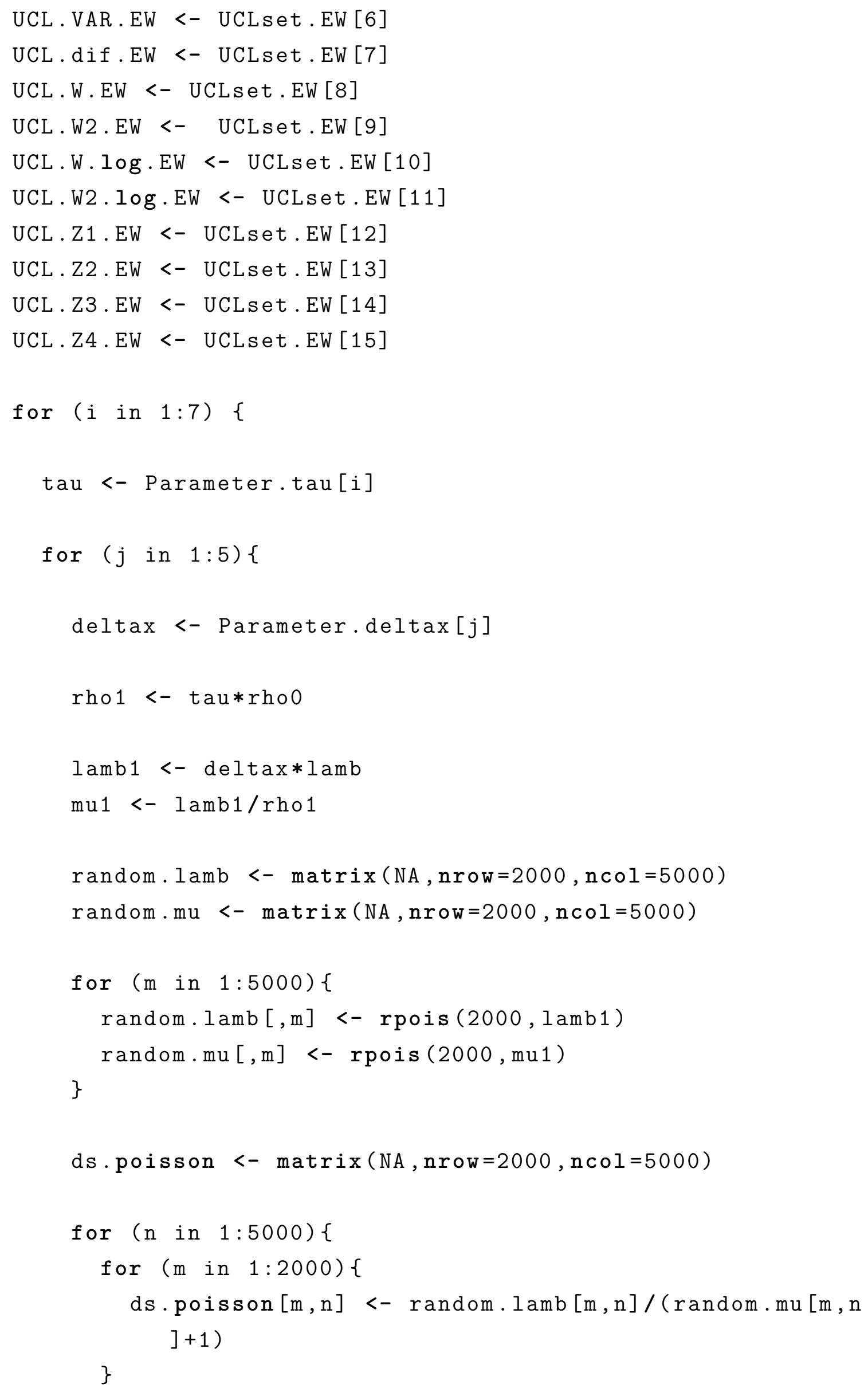




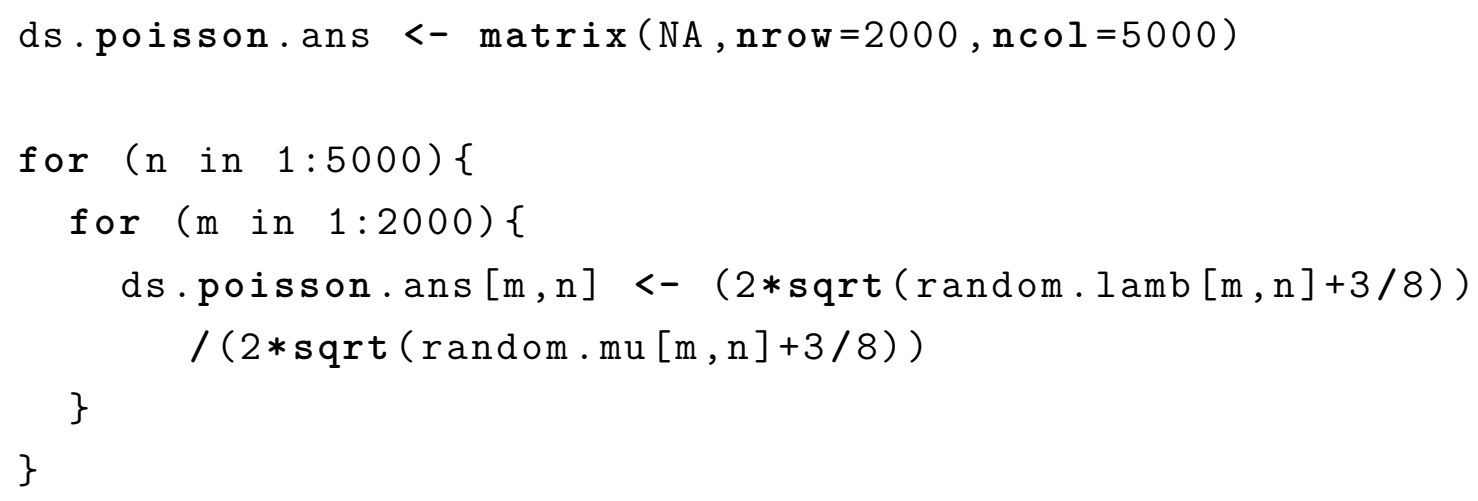




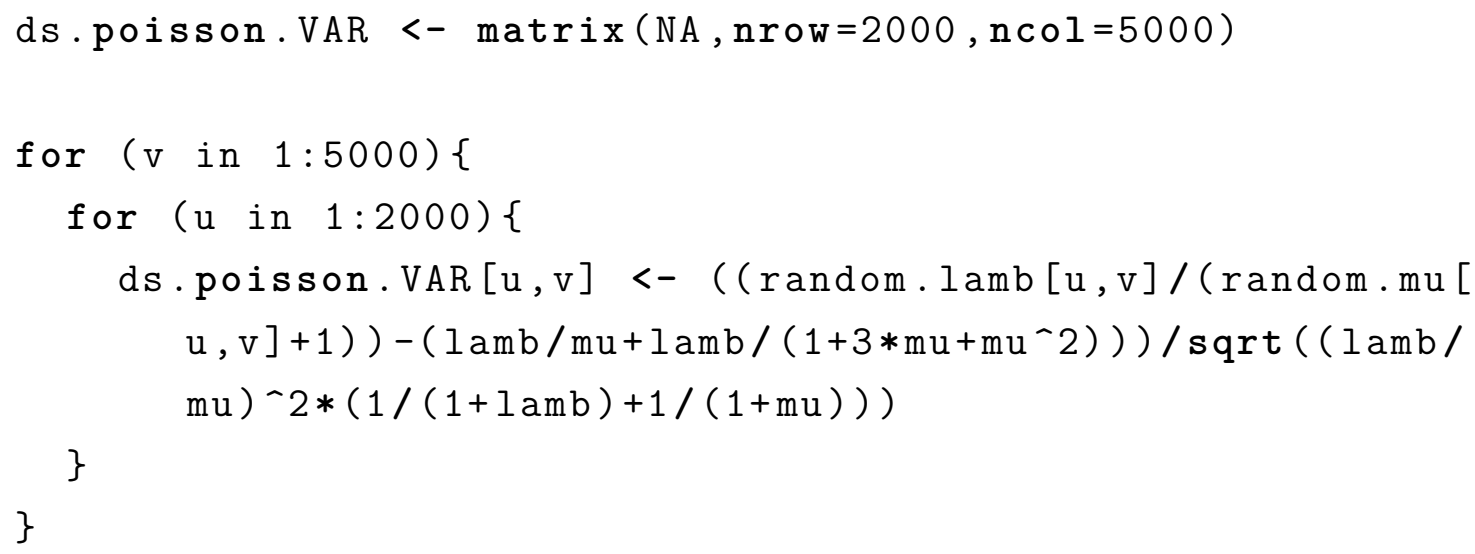




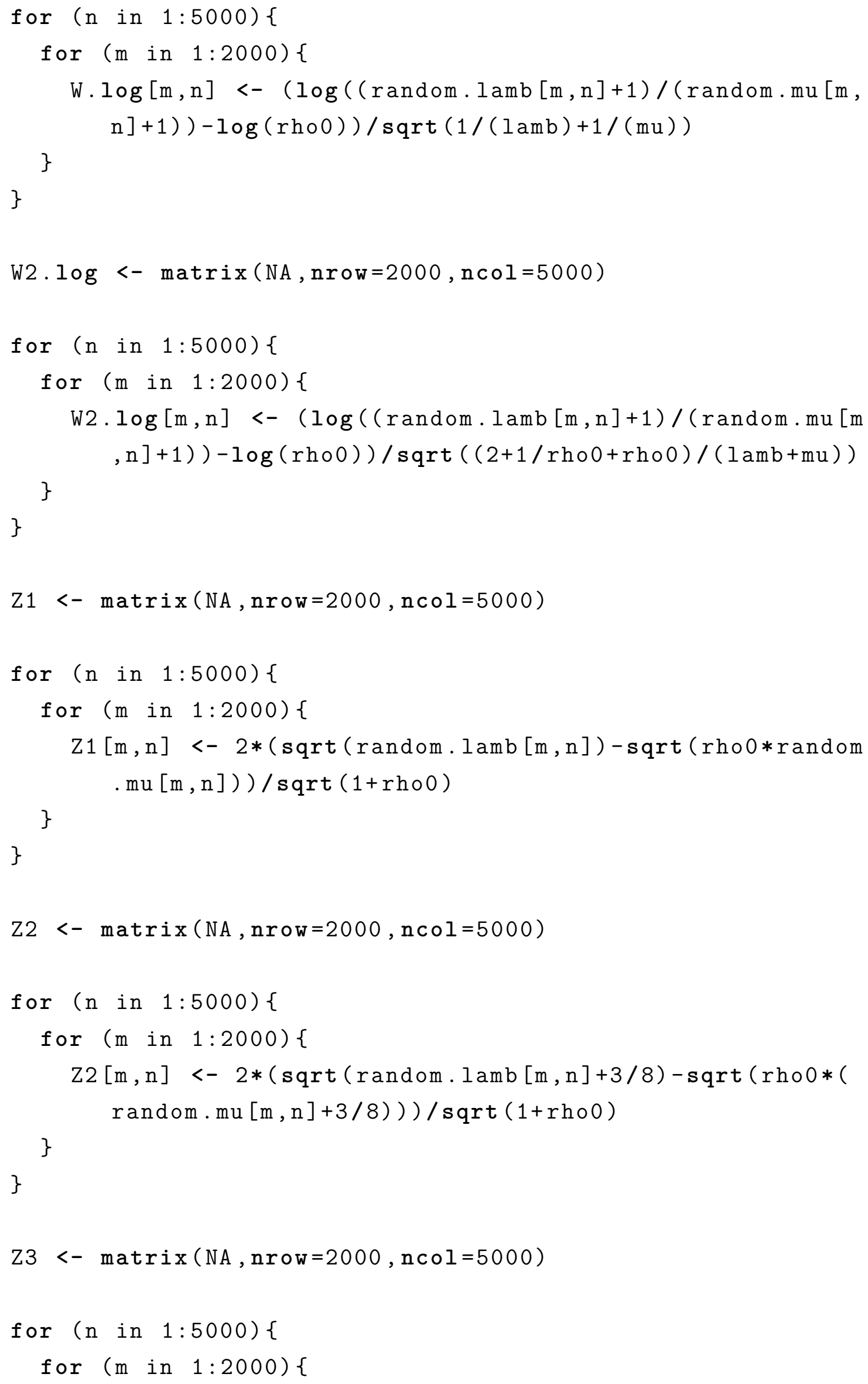




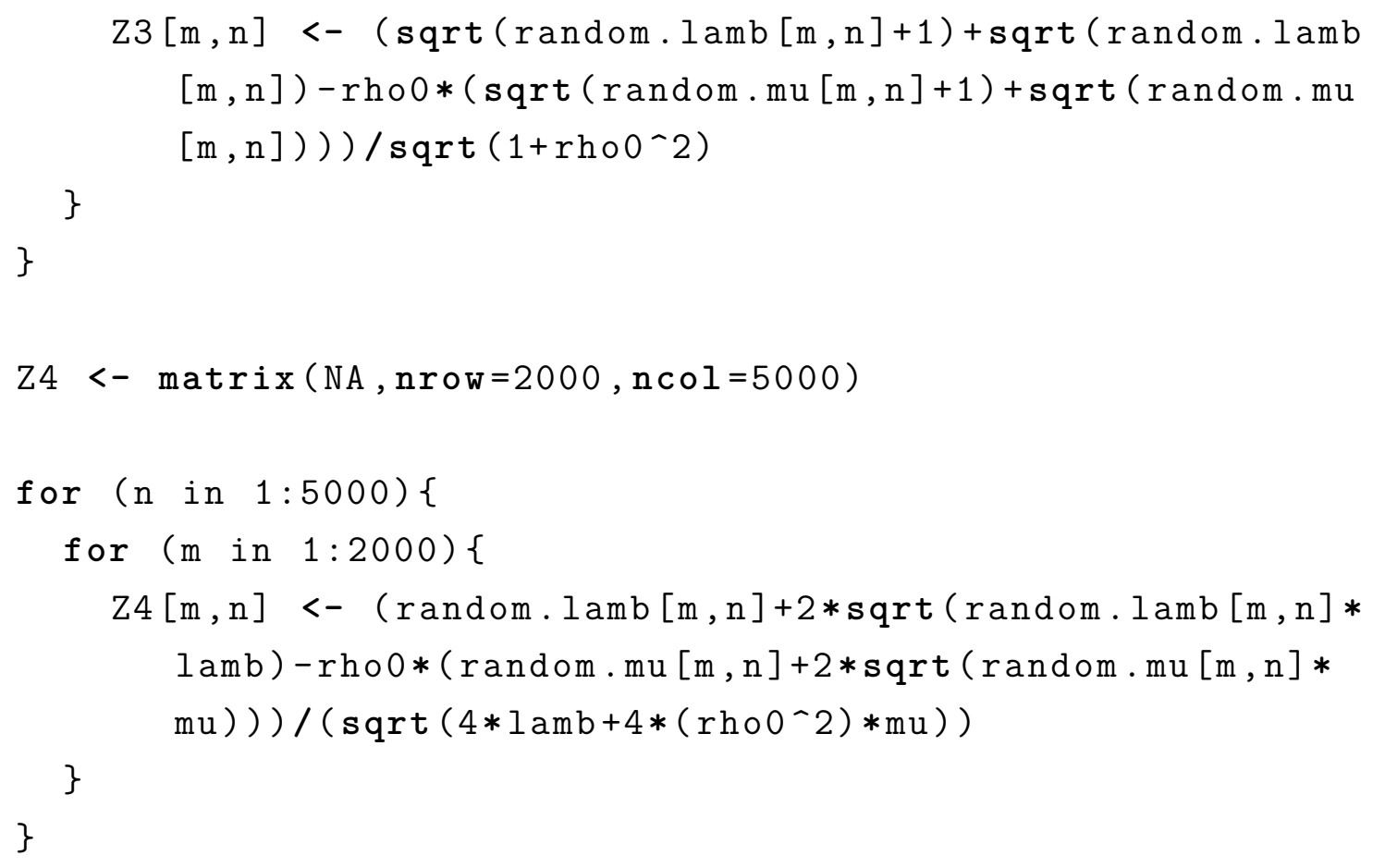


TI $<-$ NULL

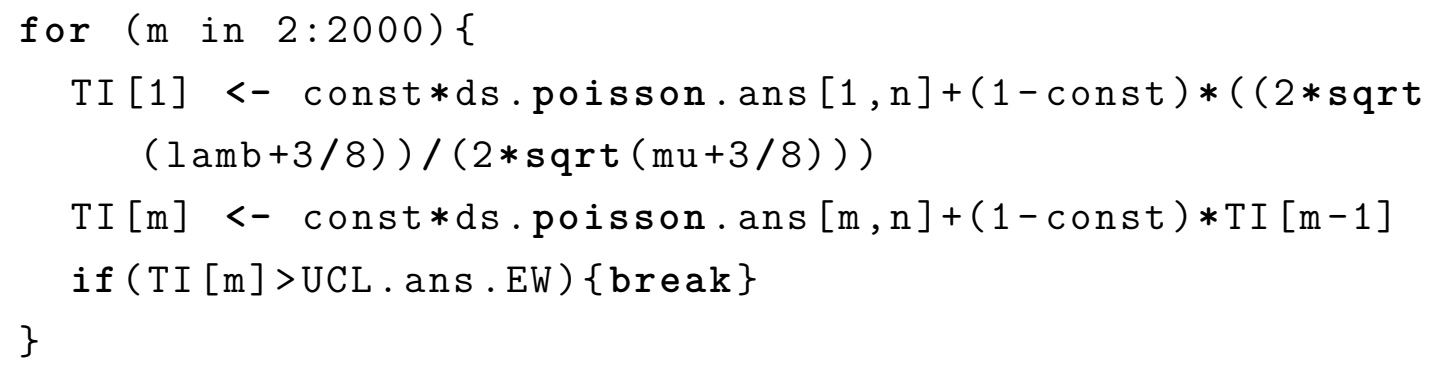




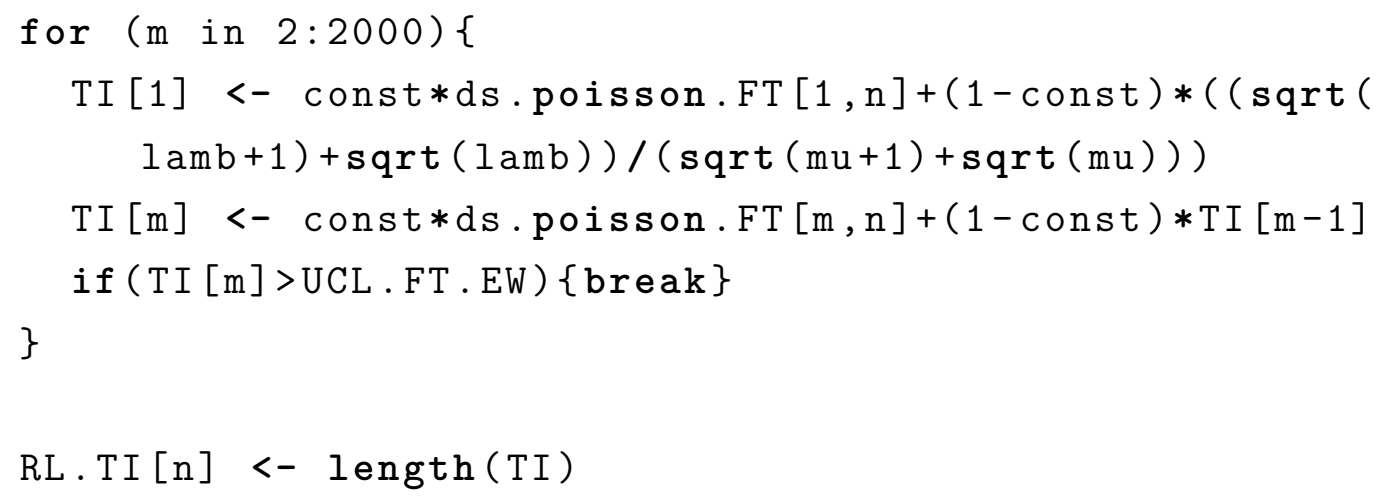




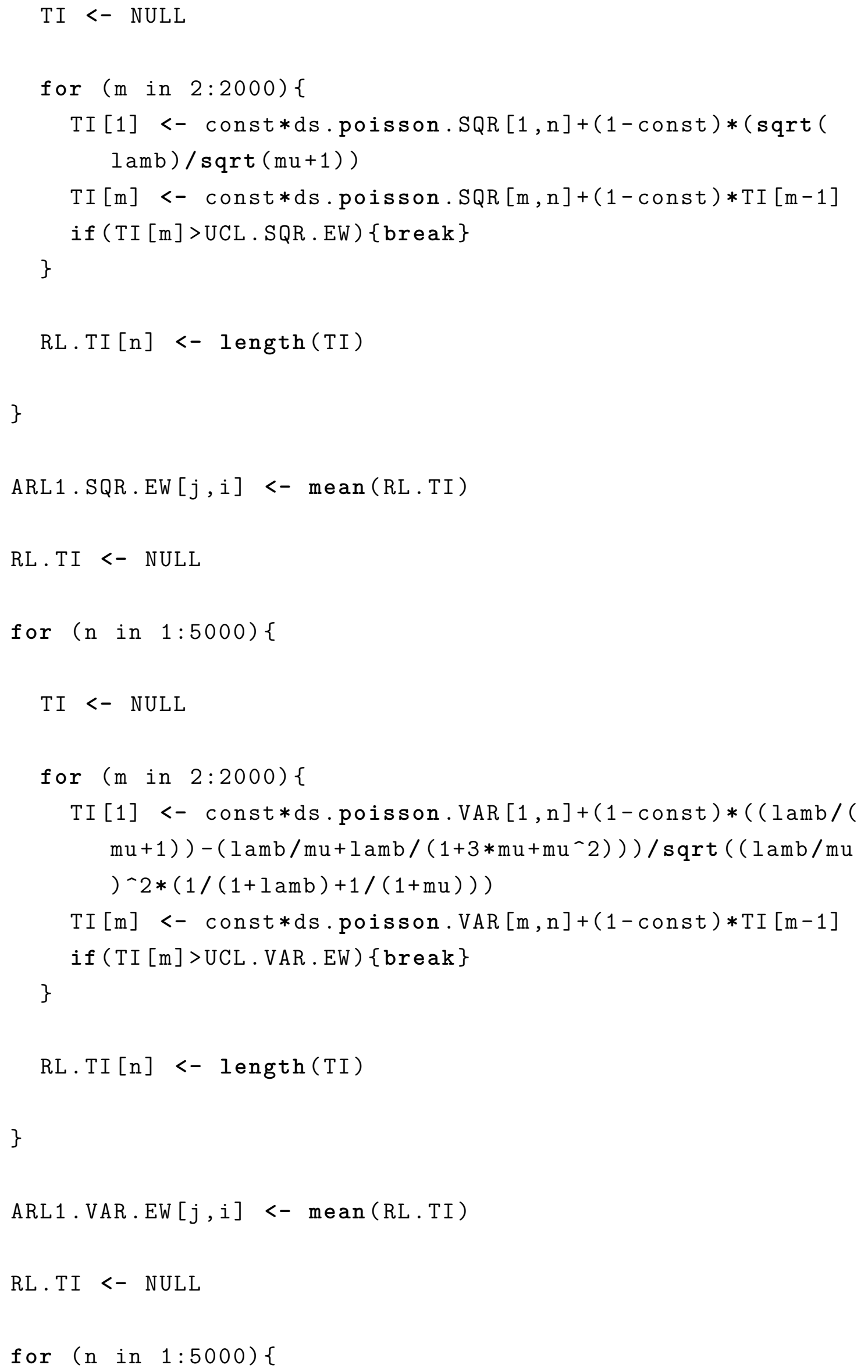




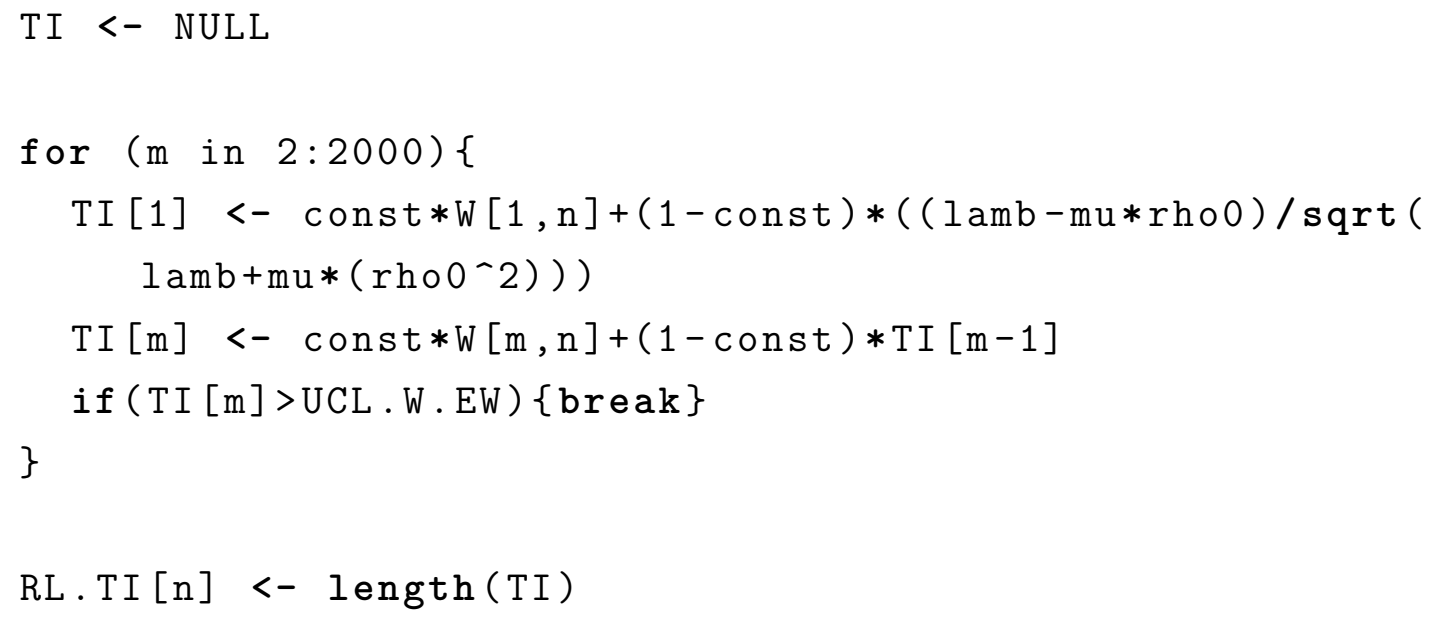


TI $<-$ NULL

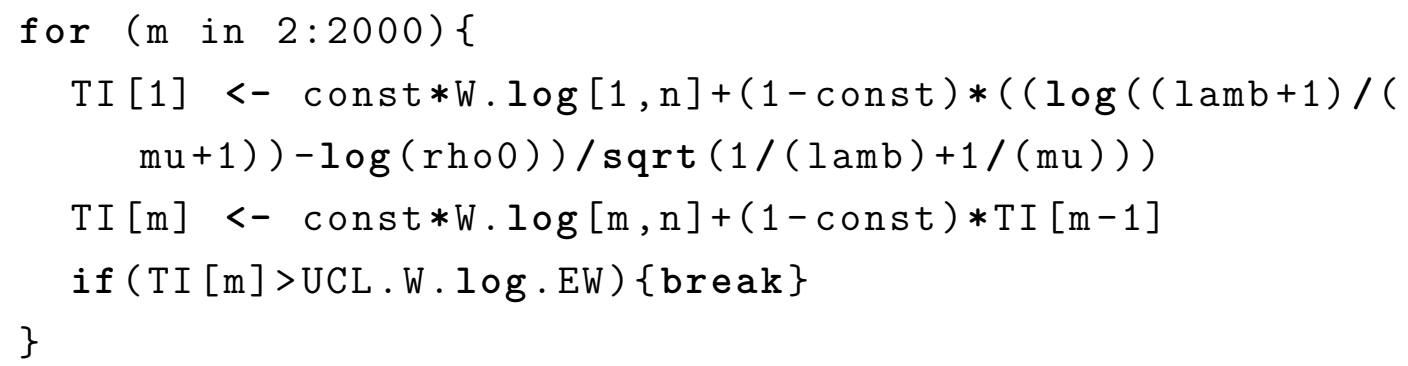




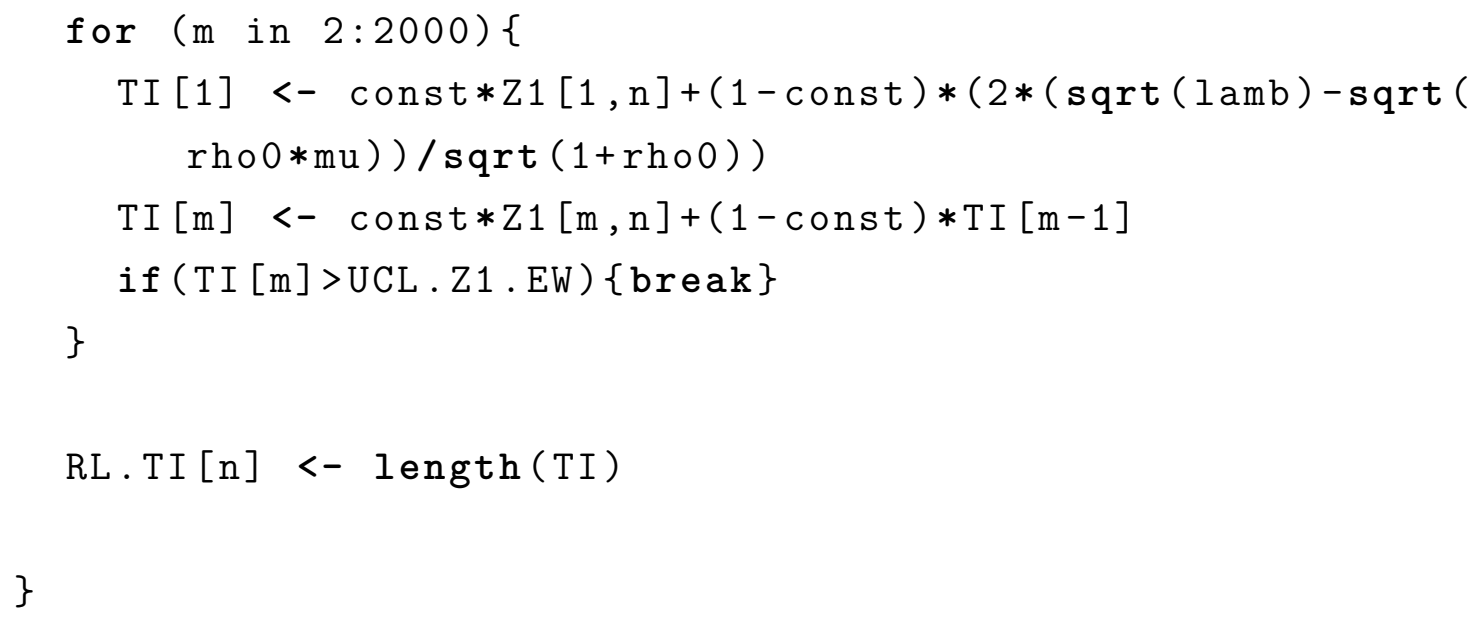




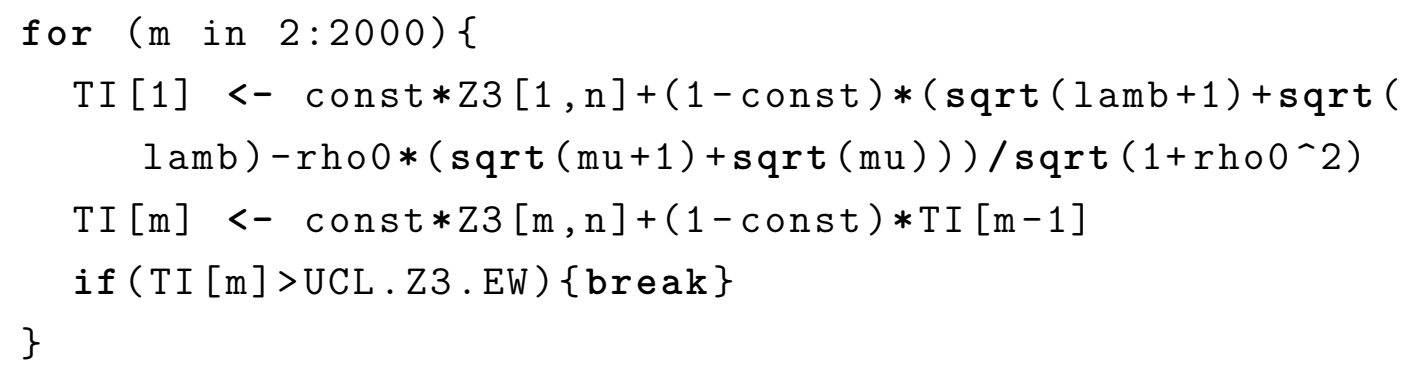

RL.TI $[n]<-$ length (TI)

\}

ARL1.Z3.EW $[j, i]<-\operatorname{mean}(R L . T I)$

RL. TI $<-$ NULL

for $(\mathrm{n}$ in $1: 5000)\{$

TI $<-N U L L$

for $(m$ in $2: 2000)\{$

TI $[1]<-$ const $* Z 4[1, n]+(1$ - const $) *((1 \mathrm{amb}+2 * \operatorname{sqrt}(1 \mathrm{amb} *$ $l \mathrm{amb})-\mathrm{rho} 0 *(\mathrm{mu}+2 * \operatorname{sqrt}(\mathrm{mu} * \mathrm{mu}))) /(\operatorname{sqrt}(4 * \mathrm{lamb}+4 *($ rho0 -2)*mu)))

TI $[\mathrm{m}]<-$ const $* \mathrm{Z} 4[\mathrm{~m}, \mathrm{n}]+(1-$ const $) * \mathrm{TI}[\mathrm{m}-1]$

if ( TI $[\mathrm{m}]>\mathrm{UCL} . \mathrm{Z} 4 . \mathrm{EW})\{$ break $\}$

\}

RL.TI $[n]<-$ length (TI)

\}

ARL1.Z4.EW $[j, i]<-$ mean (RL.TI)

\}

\}

ARL1.sd.ajt.EW <- c(ARL1.sd.EW [,1],ARL1.sd.EW [,2], ARL1.sd.EW [ , 3], ARL1.sd.EW [ , 4], ARL1.sd.EW [ ,5], ARL1.sd.EW [ ,6], ARL1.sd . EW $[, 7]$ ) 
ARL1.ans.ajt.EW <- c(ARL1.ans.EW [, 1], ARL1.ans.EW [, 2], ARL1. ans .EW [ , 3] , ARL1 . ans.EW [ , 4], ARL1 . ans.EW [ , 5] , ARL1 . ans . EW [ , 6] , ARL1 . ans. EW $[, 7]$ )

ARL1.dif.ajt.EW <- c(ARL1.dif.EW [,1], ARL1.dif.EW [,2], ARL1.dif .EW [ , 3], ARL1.dif.EW [ , 4], ARL1.dif.EW [, 5], ARL1.dif.EW [, 6], ARL1.dif.EW $[, 7])$

ARL1.FT.ajt.EW <- c(ARL1.FT.EW [, 1], ARL1.FT.EW [, 2], ARL1.FT.EW [ , 3], ARL1.FT.EW [ , 4], ARL1.FT.EW [ , 5], ARL1.FT.EW [ , 6], ARL1.FT . $\mathrm{EW}[, 7])$

ARL1.Rossi.ajt.EW <- c(ARL1.Rossi.EW [,1], ARL1.Rossi.EW [, 2], ARL1.Rossi.EW [ , 3] , ARL1 . Rossi.EW [ ,4] , ARL1.Rossi.EW [ ,5] , ARL1 .Rossi.EW $[, 6], A R L 1 . \operatorname{Rossi.EW}[, 7])$

ARL1.SQR.ajt.EW <- c(ARL1.SQR.EW [, 1], ARL1.SQR.EW [,2], ARL1.SQR .EW [ , 3], ARL1.SQR .EW [ , 4], ARL1.SQR . EW [ , 5], ARL1.SQR . EW [ , 6] , ARL1.SQR . EW $[, 7]$ )

ARL1.VAR. ajt.EW $<-c(A R L 1 . V A R . E W[, 1], A R L 1 . V A R . E W[, 2], A R L 1 . V A R$ .EW $[, 3], A R L 1 . \operatorname{VAR} . \operatorname{EW}[, 4], \operatorname{ARL} 1 . \operatorname{VAR} . \operatorname{EW}[, 5], \operatorname{ARL} 1 . \operatorname{VAR} . \operatorname{EW}[, 6]$, ARL1.VAR .EW $[, 7]$ )

ARL1.W.ajt.EW <- c(ARL1.W.EW [, 1], ARL1.W.EW [, 2], ARL1.W.EW [, 3], ARL1.W.EW [ , 4], ARL1.W.EW [ ,5], ARL1.W.EW [ , 6], ARL1.W.EW [ , 7]) ARL1.W2.ajt.EW $<-c(A R L 1$.W2.EW [, 1], ARL1.W2.EW [, 2], ARL1.W2.EW [ , 3], ARL1.W2.EW [ , 4], ARL1.W2.EW [ , 5], ARL1.W2.EW [ , 6], ARL1.W2 . EW $[, 7]$ )

ARL1.W.log.ajt.EW <- c(ARL1.W.log.EW [,1],ARL1.W.log.EW [, 2], ARL1.W. $\log . E W[, 3], A R L 1 . W . \log . E W[, 4], A R L 1 . W . \log . E W[, 5], A R L 1$

.W. $\log . \mathrm{EW}[, 6], \mathrm{ARL} 1 . \mathrm{W} . \log \cdot \operatorname{EW}[, 7])$

ARL1.W2. $\log \cdot$ ajt. EW $<-c(A R L 1 . W 2.10 g . E W[, 1], A R L 1 . W 2.10 g . E W$

$[, 2], A R L 1 . W 2 . \log . E W[, 3], A R L 1 . W 2.1 \circ g . E W[, 4], A R L 1 . W 2.1 \circ g . E W$

$[, 5], A R L 1 . W 2 \cdot \log \cdot \operatorname{EW}[, 6], A R L 1 \cdot W 2 \cdot \log \cdot \operatorname{EW}[, 7])$

ARL1.Z1.ajt.EW <- c(ARL1.Z1.EW [, 1], ARL1.Z1.EW [, 2], ARL1.Z1.EW

[ , 3], ARL1.Z1.EW [ , 4], ARL1.Z1.EW [ , 5], ARL1.Z1.EW [ , 6], ARL1.Z1.

$\mathrm{EW}[, 7]$ )

ARL1.Z2.ajt.EW <- c(ARL1.Z2.EW [, 1], ARL1.Z2.EW [, 2], ARL1.Z2.EW

[ , 3], ARL1.Z2.EW [ , 4], ARL1.Z2.EW [ , 5], ARL1.Z2.EW [ , 6], ARL1.Z2 .

EW $[, 7]$ )

ARL1.Z3.ajt.EW <- c(ARL1.Z3.EW [, 1], ARL1.Z3.EW [, 2], ARL1.Z3.EW

[ , 3], ARL1.Z3.EW [ , 4], ARL1.Z3.EW [ , 5], ARL1.Z3.EW [ , 6], ARL1.Z3.

EW $[, 7]$ )

ARL1.Z4.ajt.EW $<-c(A R L 1 . Z 4 . E W[, 1], A R L 1 . Z 4 . E W[, 2], A R L 1 . Z 4 . E W$ 
[ , 3], ARL1.Z4.EW [ , 4], ARL1.Z4.EW [ , 5], ARL1.Z4.EW [ , 6], ARL1.Z4 . EW $[, 7]$ )

Result <- matrix (c (ARL1.sd.ajt.EW, ARL1.SQR.ajt.EW, ARL1.ans . ajt.EW, ARL1.FT.ajt.EW, ARL1.Rossi.ajt.EW, ARL1.VAR.ajt.EW, ARL1.dif.ajt.EW, ARL1.W.ajt.EW, ARL1.W2.ajt.EW, ARL1.W.1og. ajt.EW, ARL1.W2.log.ajt.EW, ARL1.Z1.ajt.EW, ARL1.Z2.ajt.EW, ARL1.Z3.ajt.EW, ARL1.Z4.ajt.EW), nrow=35, ncol=15, byrow=FALSE )

$<$ ARL1 Estimation for EWMA-type control chart in case $\lambda_{10}$ and $\lambda_{20}$ are unknown>

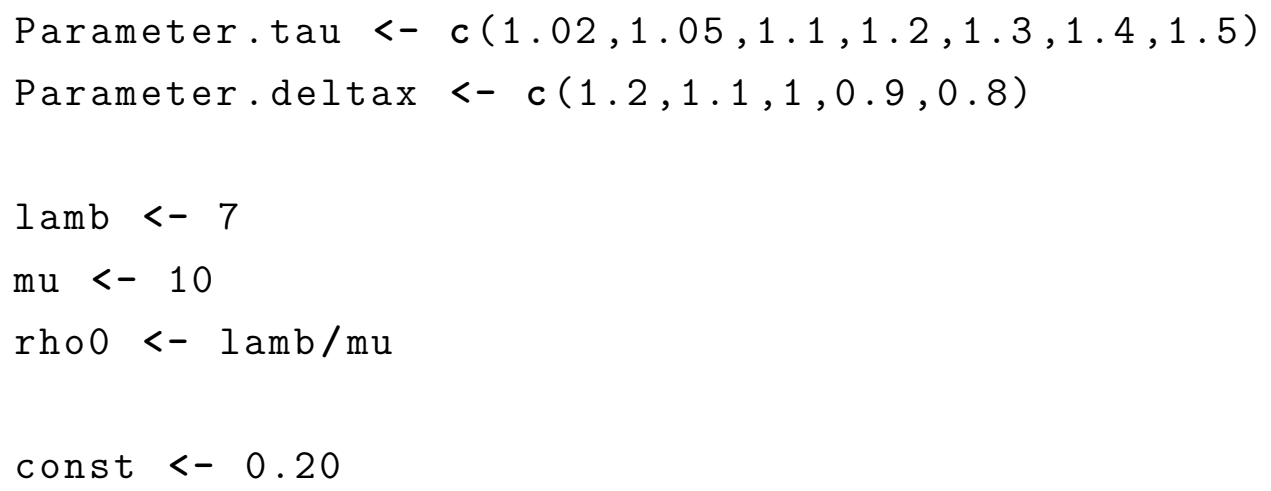

UCLset.EW <- c(1.04798, $0.98399,1.05389,1.0523,1.11371$, $-0.17911,0.10567,0.6745,0.73371,0.78192,0.76359$, $0.75087,0.76307,1.48459,2.42239)$

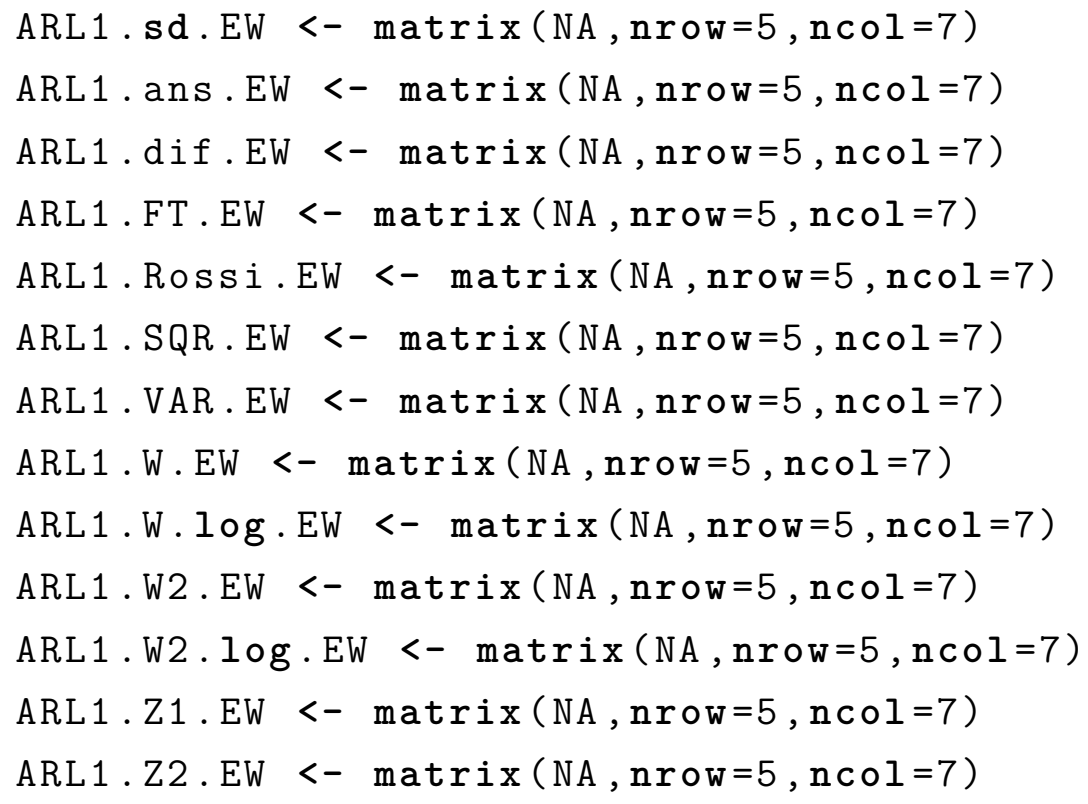




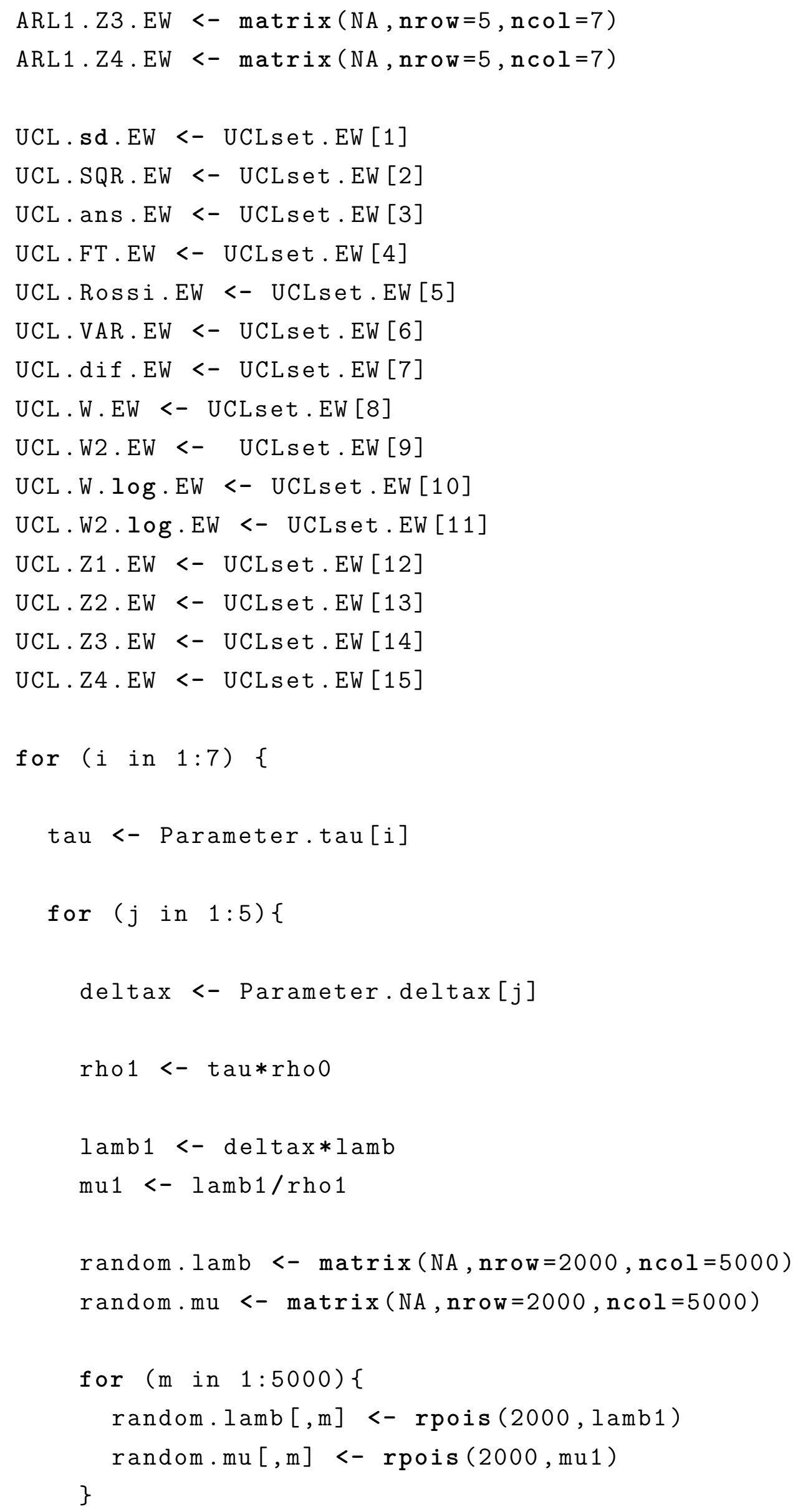




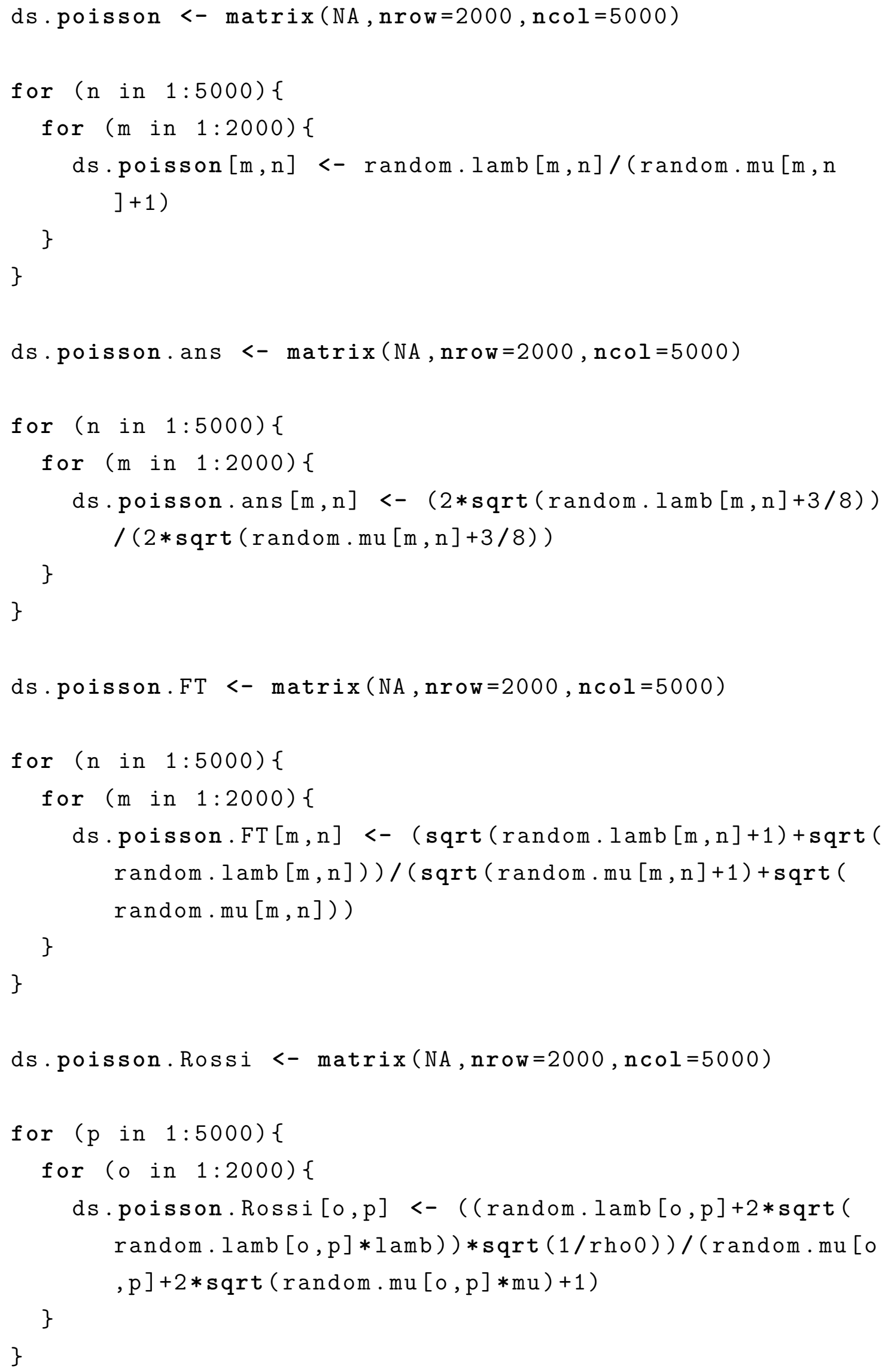




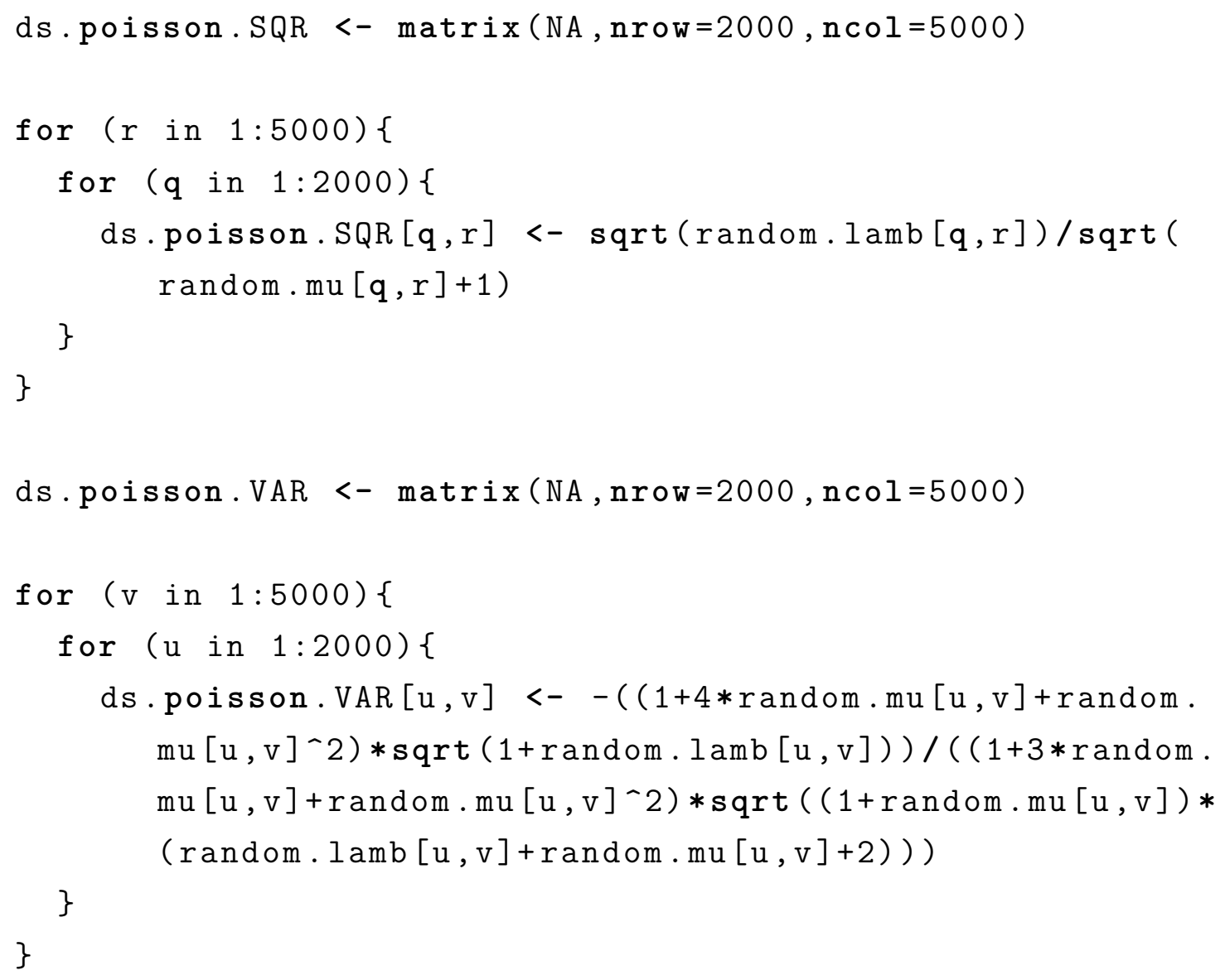




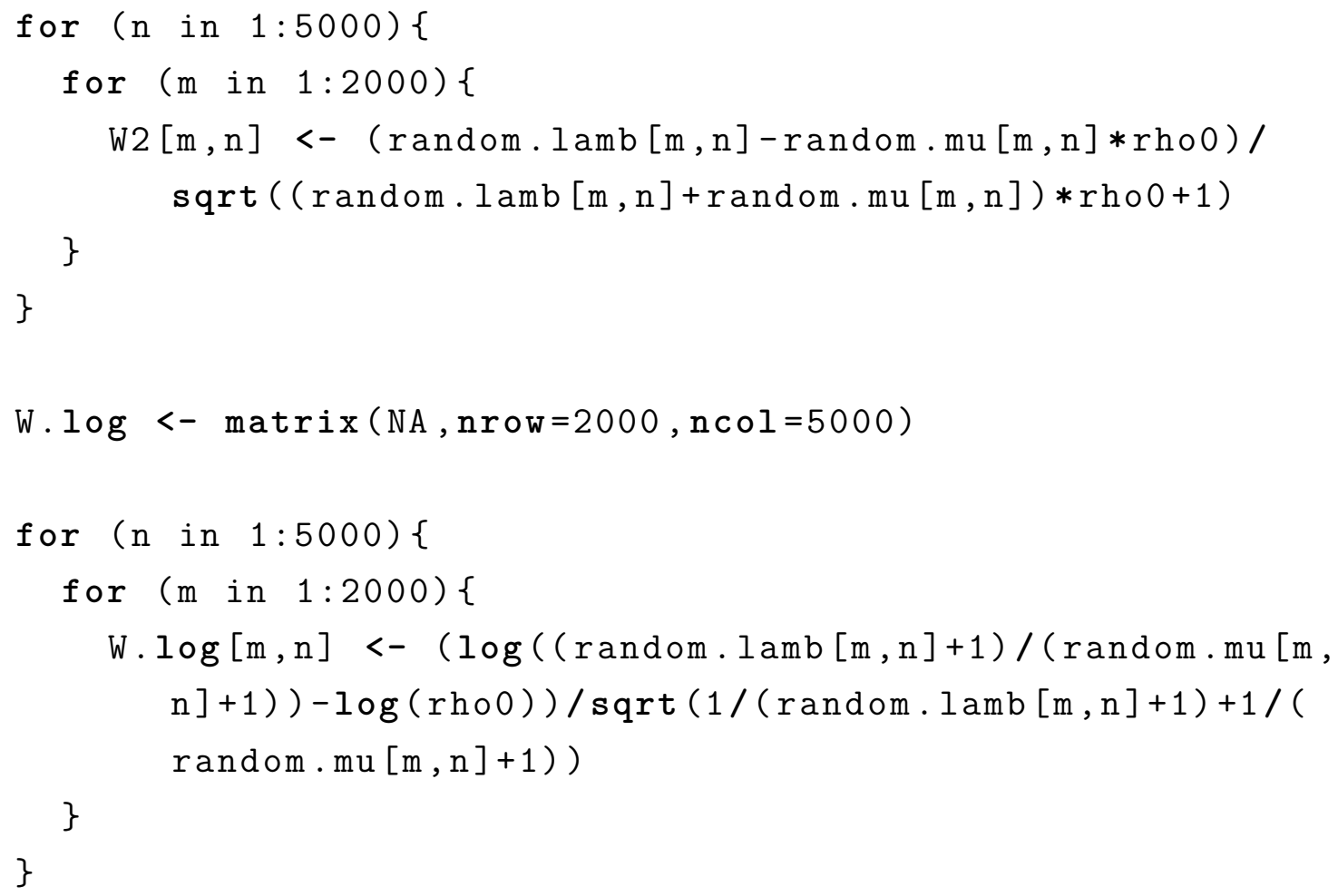




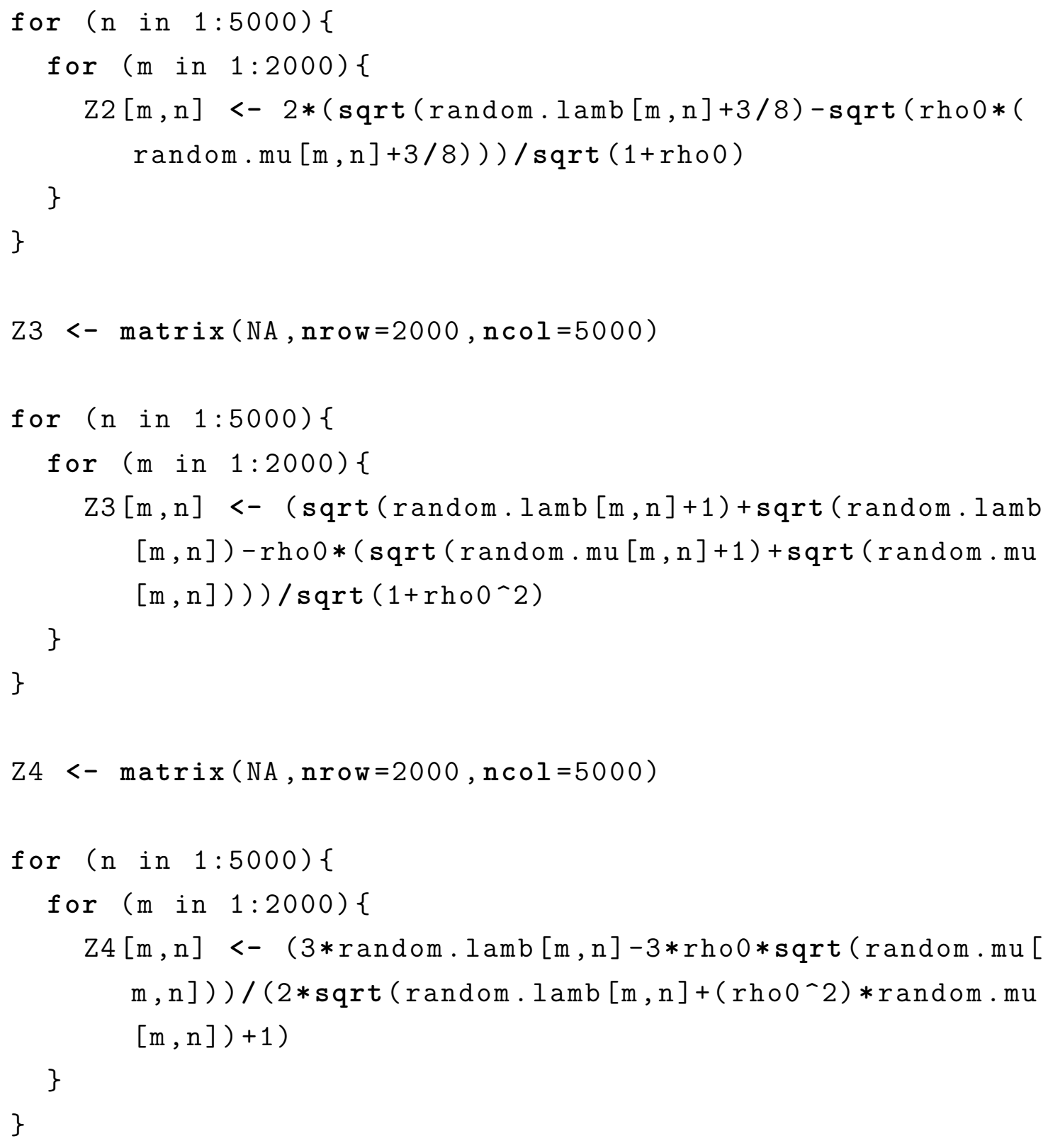




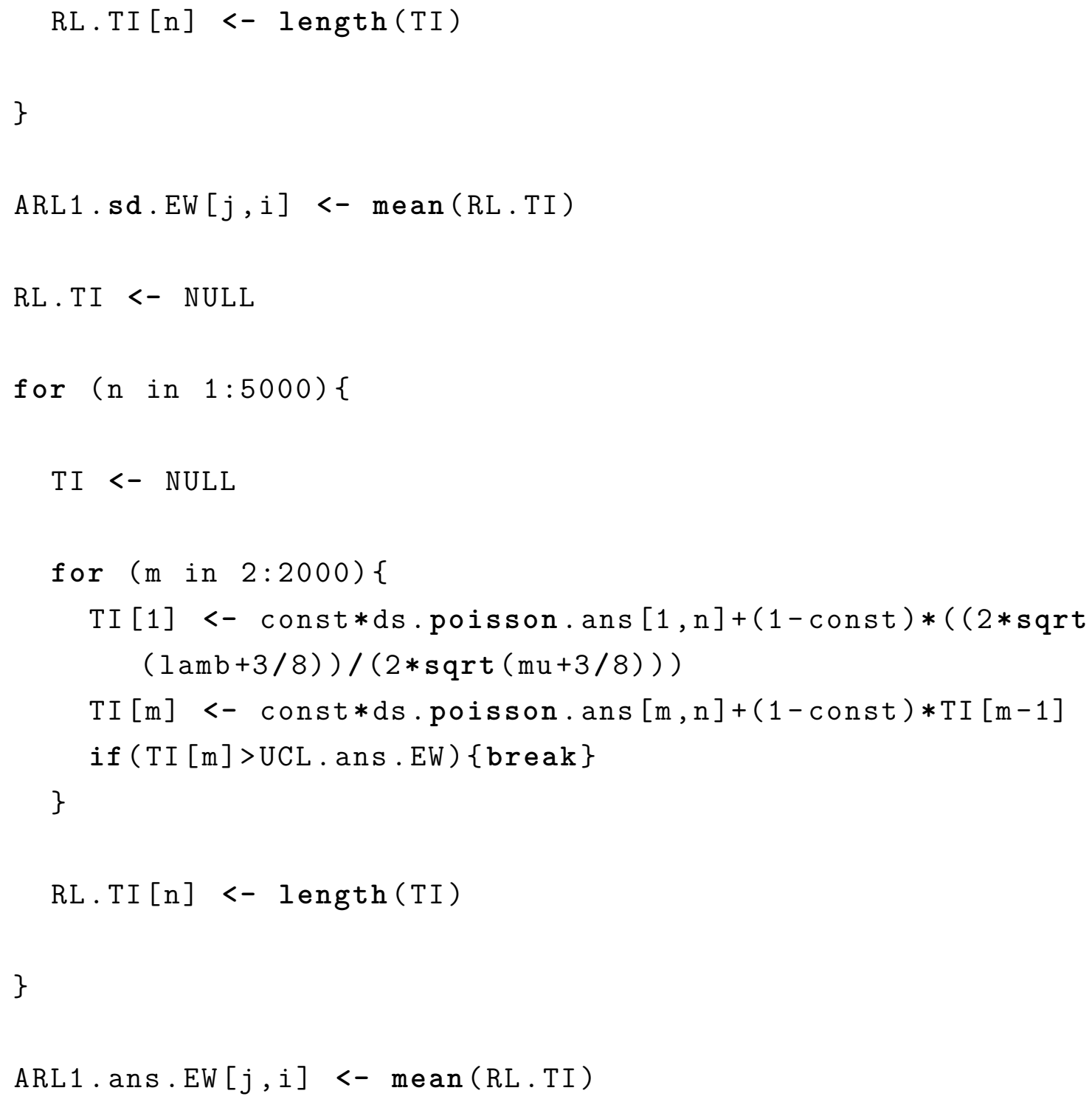




\section{RL.TI $[\mathrm{n}]<-$ length (TI)}

\}

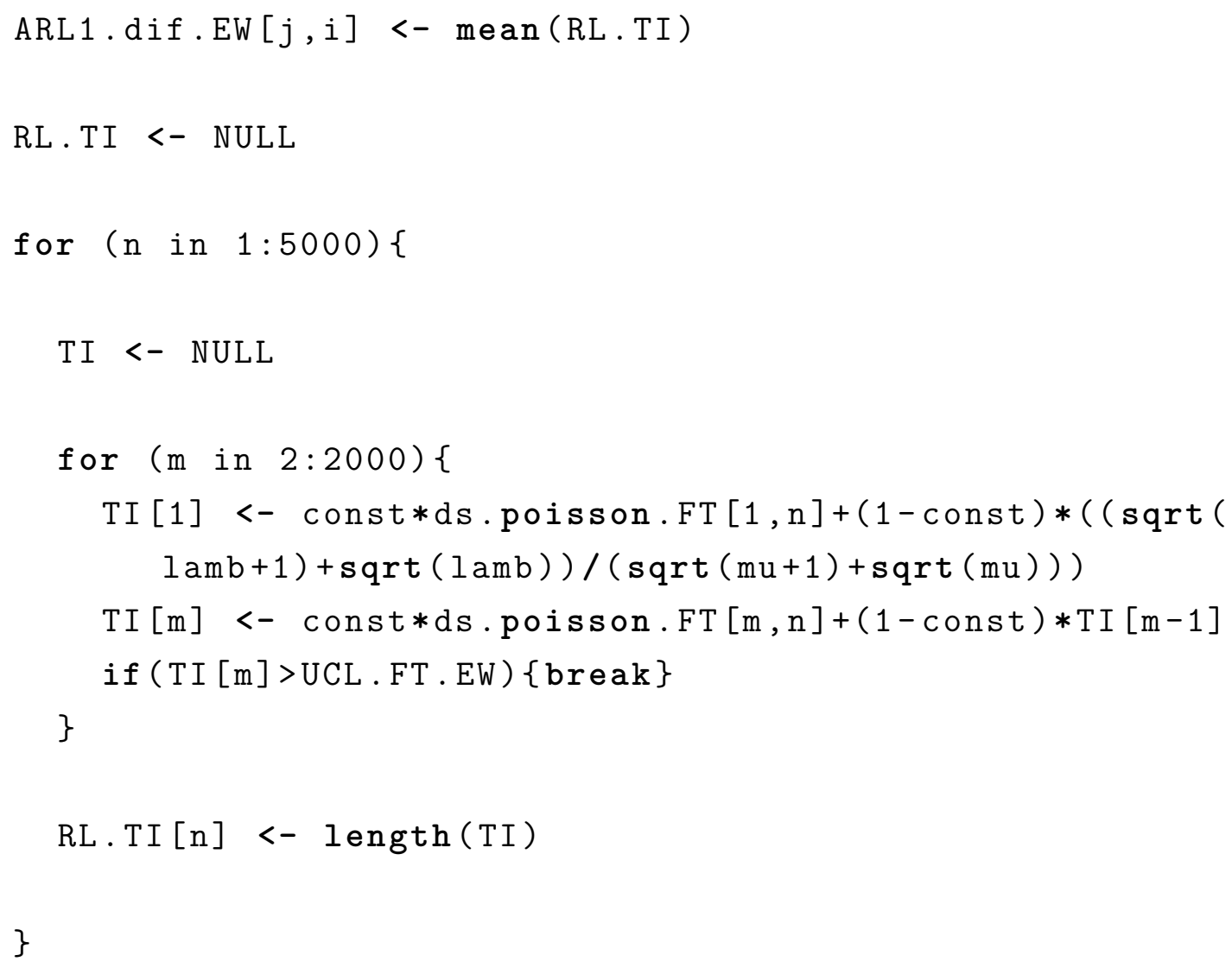




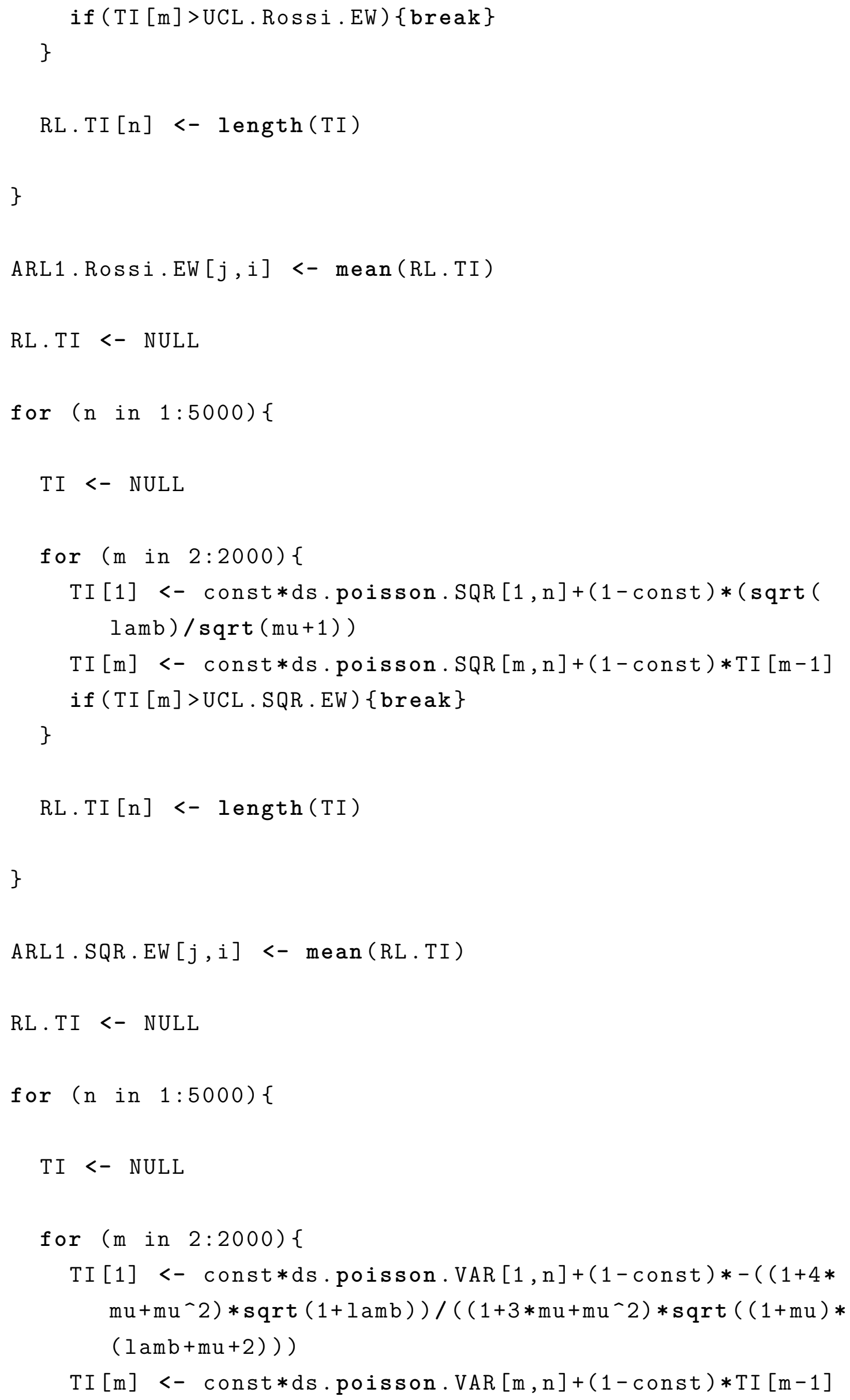




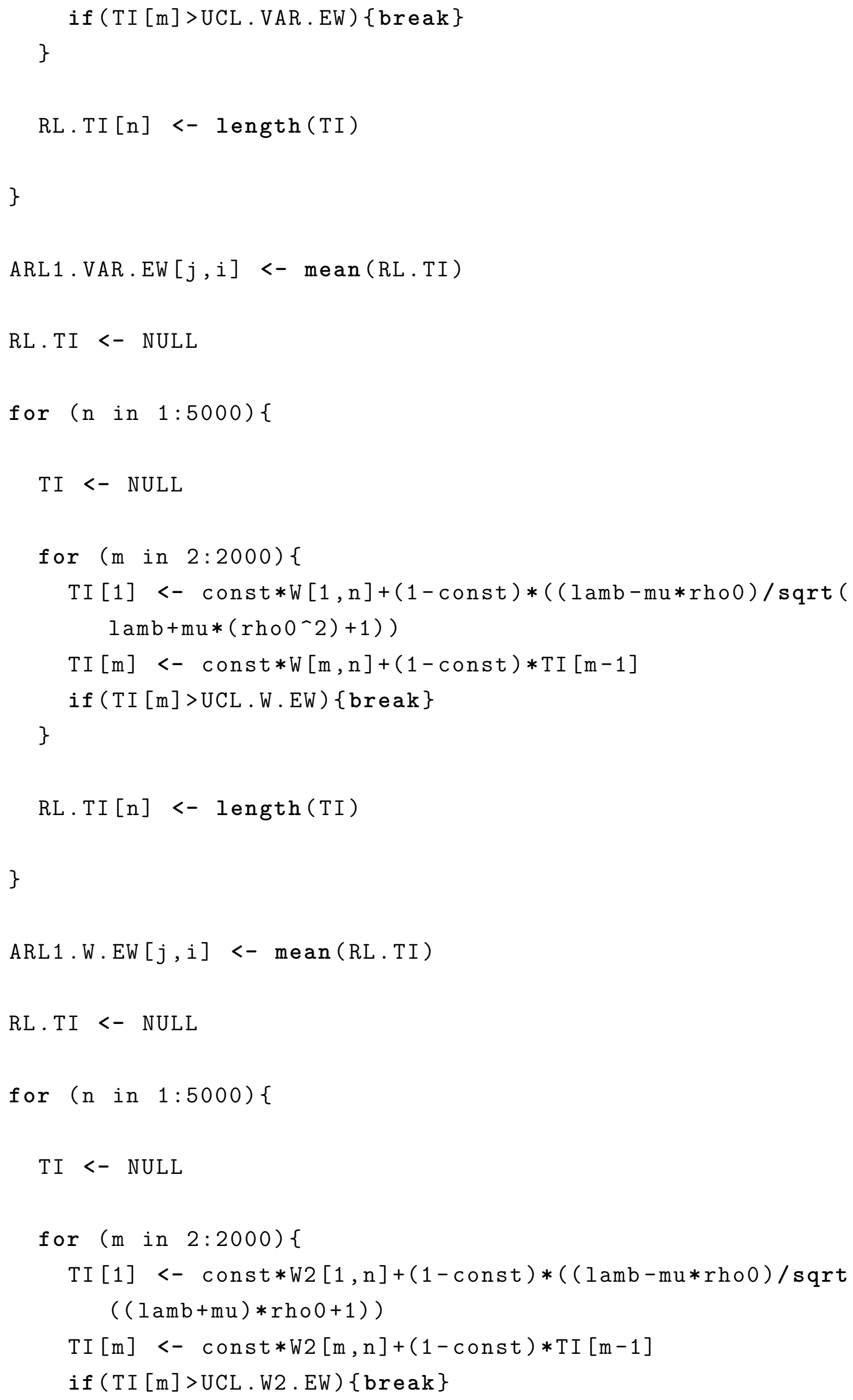


RL.TI $[n]<-$ length $(\mathrm{TI})$

\}

ARL1.W2.EW $[j, i]<-\operatorname{mean}(\mathrm{RL} . \mathrm{TI})$

RL. TI $<-$ NULL

for $(n$ in $1: 5000)\{$

TI $<-$ NULL

for $(m$ in $2: 2000)$ \{

TI $[1]<-$ const $* W . \log [1, \mathrm{n}]+(1-$ const $) *((\log ((\operatorname{lamb}+1) /($ $m u+1))-\log (r h \circ 0)) / \operatorname{sqrt}(1 /(\operatorname{lamb}+1)+1 /(m u+1)))$

TI $[\mathrm{m}]<-$ const $* W . \log [\mathrm{m}, \mathrm{n}]+(1-$ const $) * \mathrm{TI}[\mathrm{m}-1]$

if ( TI $[\mathrm{m}]>\mathrm{UCL} . \mathrm{W} . \log . \mathrm{EW})\{$ break $\}$

\}

RL.TI $[n]<-$ length (TI)

\}

ARL1.W. log.EW $[j, i]<-\operatorname{mean}(R L . T I)$

RL. TI $<-$ NULL

for $(\mathrm{n}$ in $1: 5000)\{$

TI $<-$ NULL

for $(m$ in $2: 2000)$ \{

TI $[1]<-$ const $* W 2 . \log [1, \mathrm{n}]+(1-$ const $) *((\log ((\operatorname{lamb}+1) /($ $m u+1))-\log (r h \circ 0)) / \operatorname{sqrt}((2+1 / r h \circ 0+r h \circ 0) /(l a m b+m u+1)$ ))

TI $[\mathrm{m}]<-$ const $*$ W2 $. \log [\mathrm{m}, \mathrm{n}]+(1$-const $) * \mathrm{TI}[\mathrm{m}-1]$ if ( T I $[\mathrm{m}]>\mathrm{UCL}$. W2 . log. EW) $\{$ break $\}$ 


\section{\}}

RL.TI $[\mathrm{n}]<-$ length (TI)

\}

ARL1.W2. $\log \cdot \operatorname{EW}[j, i]<-\operatorname{mean}(R L . T I)$

RL. TI $<-$ NULL

for $(n$ in $1: 5000)\{$

TI <- NULL

for $(m$ in $2: 2000)\{$

TI $[1]<-$ const $* \mathrm{Z} 1[1, \mathrm{n}]+(1-$ const $) *(2 *(\operatorname{sqrt}($ lamb $)-\operatorname{sqrt}($ $r h \circ 0 * m u)) / \operatorname{sqrt}(1+r h \circ 0))$

$\mathrm{TI}[\mathrm{m}]<-$ const $* \mathrm{Z} 1[\mathrm{~m}, \mathrm{n}]+(1-$ const $) * \mathrm{TI}[\mathrm{m}-1]$

if ( TI $[\mathrm{m}]>\mathrm{UCL} . \mathrm{Z1}$. EW $)\{$ break

\}

RL.TI $[\mathrm{n}]<-$ length (TI)

\}

ARL1.Z1.EW $[j, i]<-\operatorname{mean}(R L . T I)$

RL.TI $<-N U L L$

for $(n$ in $1: 5000)\{$

TI $<-$ NULL

for $(m$ in $2: 2000)\{$

TI $[1]<-$ const $* \mathrm{Z} 2[1, \mathrm{n}]+(1-$ const $) *(2 *(\operatorname{sqrt}(1 \mathrm{amb}+3 / 8)-$ $\operatorname{sqrt}(\operatorname{rho0} *(\mathrm{mu}+3 / 8))) / \operatorname{sqrt}(1+\operatorname{rho0}))$

$\mathrm{TI}[\mathrm{m}]<-$ const $* \mathrm{Z} 2[\mathrm{~m}, \mathrm{n}]+(1-$ const $) * \mathrm{TI}[\mathrm{m}-1]$

if ( TI $[\mathrm{m}]>\mathrm{UCL} . \mathrm{Z2}$. EW) $\{$ break $\}$

\} 


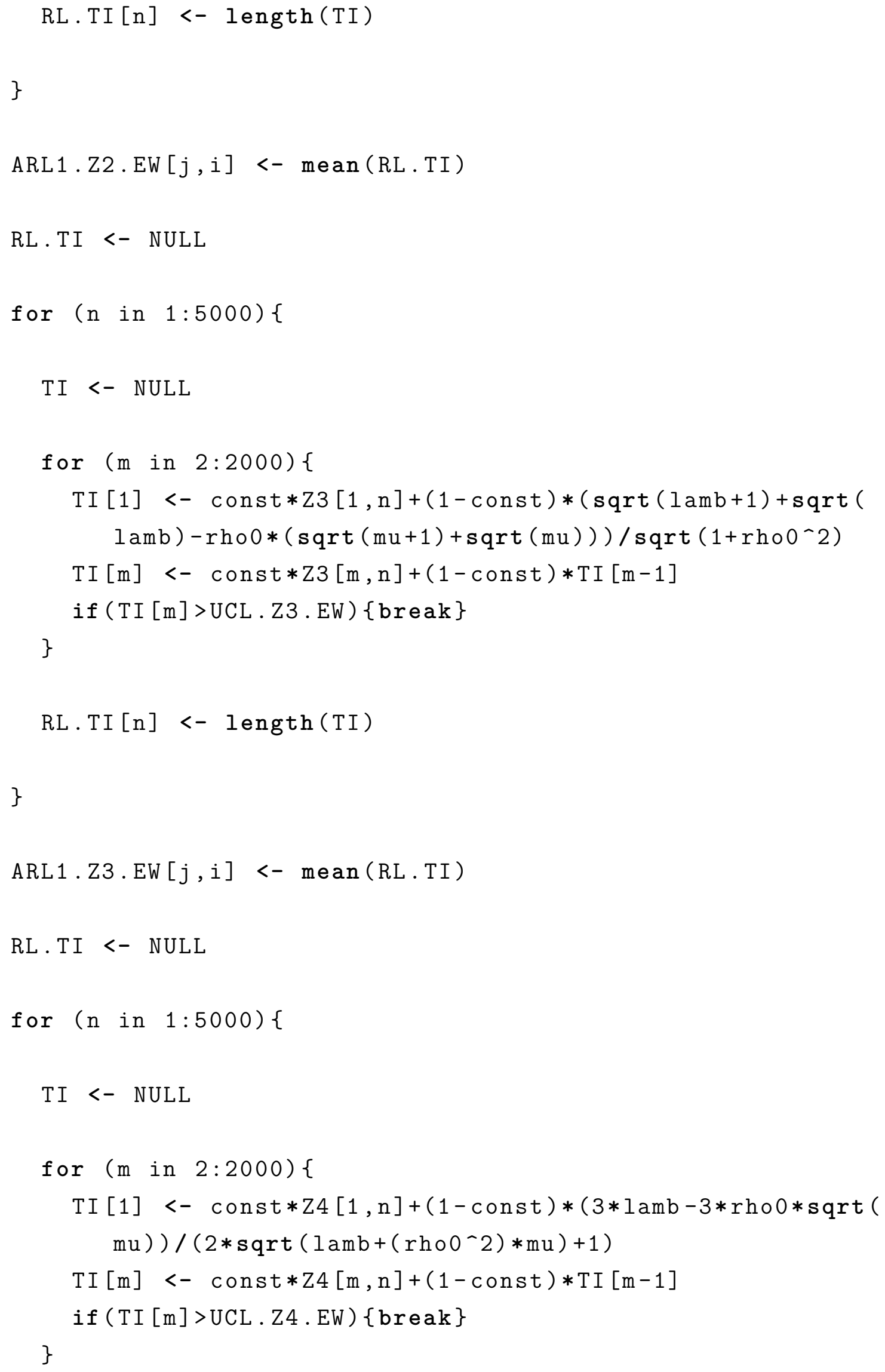




\author{
RL.TI $[\mathrm{n}]<-$ length (TI) \\ \} \\ ARL1.Z4.EW $[j, i]<-\operatorname{mean}($ RL.TI) \\ \} \\ \}
}

ARL1.sd.ajt.EW <- c(ARL1.sd.EW [,1], ARL1.sd.EW [,2], ARL1.sd.EW

[ , 3], ARL1.sd.EW [ , 4], ARL1.sd.EW [ ,5], ARL1.sd.EW [ , 6], ARL1.sd.

EW $[, 7]$ )

ARL1. ans.ajt.EW <- c(ARL1. ans.EW [, 1], ARL1. ans. EW [,2], ARL1. ans

.EW [ , 3] , ARL1 . ans.EW [ , 4], ARL1 . ans.EW [ , 5], ARL1 . ans. EW [ , 6] ,

ARL1 . ans.EW $[, 7]$ )

ARL1.dif.ajt.EW <- c(ARL1.dif.EW [,1],ARL1.dif.EW [,2], ARL1.dif

.EW [ , 3], ARL1.dif.EW [, 4], ARL1.dif.EW [, 5], ARL1.dif.EW [, 6],

ARL1.dif.EW $[, 7])$

ARL1.FT.ajt.EW $<-c(A R L 1 . F T . E W[, 1], A R L 1 . F T . E W[, 2], A R L 1 . F T . E W$

[ , 3], ARL1.FT.EW [ , 4], ARL1.FT.EW [ , 5], ARL1.FT.EW [ , 6], ARL1.FT .

EW $[, 7]$ )

ARL1.Rossi.ajt.EW - c(ARL1.Rossi.EW[,1],ARL1.Rossi.EW [,2],

ARL1.Rossi.EW[, 3], ARL1.Rossi.EW [, 4], ARL1.Rossi.EW[, 5], ARL1

.Rossi.EW $[, 6]$, ARL1.Rossi.EW $[, 7])$

ARL1.SQR. ajt.EW - c (ARL1.SQR.EW [, 1], ARL1.SQR.EW [, 2], ARL1.SQR

.EW [ , 3], ARL1.SQR .EW [ , 4], ARL1.SQR . EW [ , 5], ARL1.SQR . EW [ , 6],

ARL1.SQR .EW $[, 7]$ )

ARL1.VAR.ajt.EW $<-c(A R L 1 . \operatorname{VAR} . \mathrm{EW}[, 1], \operatorname{ARL} 1 . \operatorname{VAR} . \mathrm{EW}[, 2], \mathrm{ARL} 1 . \mathrm{VAR}$

.EW [ , 3], ARL1.VAR . EW [ , 4], ARL1.VAR . EW [ , 5], ARL1.VAR.EW [ ,6],

ARL1.VAR . EW $[, 7])$

ARL1.W.ajt.EW <- c(ARL1.W.EW [, 1], ARL1.W.EW [, 2], ARL1.W.EW [, 3],

ARL1.W.EW [ , 4], ARL1.W.EW [ , 5], ARL1.W.EW [ , 6], ARL1.W.EW [ , 7])

ARL1.W2. ajt. EW <- c (ARL1.W2.EW [, 1], ARL1.W2.EW [, 2], ARL1.W2.EW

[ , 3], ARL1.W2.EW [ , 4], ARL1.W2.EW [ , 5], ARL1.W2 . EW [ , 6], ARL1.W2 .

EW $[, 7]$ )

ARL1.W.log.ajt.EW $<-c(A R L 1 . W . \log . E W[, 1], A R L 1 . W .10 g . E W[, 2]$,

ARL1.W. $\log . \operatorname{EW}[, 3], A R L 1 . W . \log \cdot \operatorname{EW}[, 4], A R L 1 . W . \log \cdot \operatorname{EW}[, 5], A R L 1$

.W. $\log . \operatorname{EW}[, 6], A R L 1 . W . \log . \operatorname{EW}[, 7])$ 
ARL1.W2.log.ajt.EW <- c(ARL1.W2.log.EW [, 1], ARL1.W2.log. EW $[, 2], A R L 1 . W 2 \cdot \log \cdot \operatorname{EW}[, 3], A R L 1 . W 2 \cdot \log \cdot \operatorname{EW}[, 4], A R L 1 . W 2 . \log \cdot E W$ $[, 5], A R L 1 . W 2 \cdot \log \cdot \operatorname{EW}[, 6], A R L 1 \cdot W 2 . \log \cdot \operatorname{EW}[, 7])$

ARL1.Z1.ajt.EW <- c(ARL1.Z1.EW[,1],ARL1.Z1.EW [, 2], ARL1.Z1.EW [ , 3], ARL1.Z1.EW [ , 4], ARL1.Z1.EW [ , 5], ARL1.Z1.EW [ , 6], ARL1.Z1. $\mathrm{EW}[, 7])$

ARL1.Z2.ajt.EW <- c(ARL1.Z2.EW [, 1], ARL1.Z2.EW [, 2], ARL1.Z2.EW [ , 3], ARL1.Z2.EW [ , 4], ARL1.Z2.EW [ , 5], ARL1.Z2.EW [ , 6], ARL1.Z2 . EW $[, 7]$ )

ARL1.Z3.ajt.EW <- c(ARL1.Z3.EW [, 1], ARL1.Z3.EW [, 2], ARL1.Z3.EW [ , 3], ARL1.Z3.EW [ , 4], ARL1.Z3.EW [ , 5], ARL1.Z3.EW [ , 6], ARL1.Z3. EW $[, 7])$

ARL1.Z4.ajt.EW <- c(ARL1.Z4.EW [, 1],ARL1.Z4.EW [, 2], ARL1.Z4.EW [ , 3], ARL1.Z4.EW [ , 4], ARL1.Z4.EW [ , 5], ARL1.Z4.EW [ , 6], ARL1.Z4. EW $[, 7]$ )

Result <- matrix (c (ARL1.sd.ajt.EW, ARL1.SQR. ajt.EW, ARL1.ans. ajt.EW, ARL1.FT.ajt.EW, ARL1.Rossi.ajt.EW, ARL1.VAR.ajt.EW, ARL1.dif .ajt.EW, ARL1.W.ajt.EW, ARL1.W2.ajt.EW, ARL1.W.1og. ajt.EW, ARL1.W2. log.ajt.EW, ARL1.Z1.ajt.EW, ARL1.Z2.ajt.EW, ARL1.Z3.ajt.EW, ARL1.Z4.ajt. EW), nrow =35, ncol=15, byrow=FALSE ) 